Sengupta et al.

\title{
1 Cell lineage as a predictor of immune response in neuroblastoma
}

3 Satyaki Sengupta ${ }^{1,2, *}$, Sanjukta Das ${ }^{1,2, \star}$, Angela Crespo ${ }^{2,3}$, Brian Miller ${ }^{4,5,6,7}$, Bandana Sharma ${ }^{1,2}$,

4 Shupei Zhang ${ }^{8}$, Ruben Dries ${ }^{1,2}$, Hao Huang ${ }^{1,2}$, Malgorzata Krajewska ${ }^{1,2}$, David N. Debruyne ${ }^{1,2}$,

5 Luigi Soriano ${ }^{1}$, Malkiel A. Cohen ${ }^{8}$, Rogier Versteeg ${ }^{9}$, Rudolf Jaenisch ${ }^{8,10}$, Stefani Spranger ${ }^{10,11}$,

6 Judy Lieberman ${ }^{2,3}$, Rani E. George $\mathrm{e}^{1,2,12}$

7

$8{ }^{1}$ Department of Pediatric Oncology, Dana-Farber Cancer Institute, Boston, MA 02215, USA

92 Department of Pediatrics, Harvard Medical School, Boston, MA 02215, USA

10 3Program in Cellular and Molecular Medicine, Boston Children's Hospital, Boston, MA, USA.

$11{ }^{4}$ Broad Institute of MIT and Harvard, Cambridge, Massachusetts 02142, USA.

$12{ }^{5}$ Department of Medical Oncology, Dana-Farber Cancer Institute and Harvard Medical School,

13 Boston, Massachusetts 02215 USA.

$14{ }^{6}$ Department of Immunology, Blavatnik Institute, Harvard Medical School, Boston, MA, USA

$15 \quad{ }^{7}$ Evergrande Center for Immunological Diseases, Harvard Medical School and Brigham and

16 Women's Hospital, Boston, MA, USA.

17 'Whitehead Institute for Biomedical Research, 455 Main Street, Cambridge, MA 02142, USA

18 9Department of Oncogenomics, Amsterdam UMC University of Amsterdam, Meibergdreef 9,

191105 AZ Amsterdam, The Netherlands

$20{ }^{10}$ Department of Biology, Massachusetts Institute of Technology, Cambridge, MA 02142,

21 USA

$22{ }^{11}$ Koch Institute for Integrative Cancer Research, Cambridge, MA 02142, USA

23 *These authors contributed equally

$24 \quad{ }^{12}$ Corresponding author: rani george@dfci.harvard.edu 
Sengupta et al.

\section{SUMMARY}

28 Immunotherapy for patients with neuroblastoma has met with limited success, partly due to an

29 incomplete understanding of the mechanisms underlying immune responsiveness in this clinically

30 and genetically heterogenic tumor. Here, we undertook an unbiased analysis using dimension

31 reduction and UMAP visualization of transcriptional signatures derived from 498 primary

32 neuroblastoma tumors. Four distinct clusters based on differentially expressed genes emerged,

33 of which one, representing about $30 \%$ and comprising mainly of $M Y C N$-nonamplified tumors, was

34 notable for the high expression of genes associated with both immune response activation and

35 suppression. This capacity to elicit a productive immune response resided exclusively in tumors

36 with dominant populations of undifferentiated, neural crest-like or mesenchymal cells; by contrast,

37 tumors comprising primarily of committed, adrenergic neuron-like cells were less immunogenic.

38 Mesenchymal neuroblastoma cells were enriched for innate and adaptive immune gene

39 signatures, demonstrated engagement with cytotoxic $\mathrm{T}$ and natural killer cells, and induced

40 immune cell infiltration in an immunocompetent mouse model. Transcriptional or targeted therapy-

41 induced reprogramming of adrenergic cells to the mesenchymal state led to reactivation of tumor

42 cell-intrinsic immune genes. Key immune response genes in adrenergic tumor cells were found

43 to be epigenetically silenced by the PRC2 complex, and such repression could be relieved by

44 either mesenchymal cell state reprogramming or EZH2 inhibition, leading to increased activation

45 of natural killer cells by the tumor cells. These data identify cell lineage as a major determinant of

46 the immunogenic potential in neuroblastoma that could be used to stratify patients who are most

47 likely to benefit from immunotherapy. 
Sengupta et al.

\section{INTRODUCTION}

The anti-disialoganglioside GD2 monoclonal antibody dinutuximab has significantly improved event free survival rates in neuroblastoma ${ }^{1}$. Derived from the developing neural crest, this common solid tumor of childhood manifests as an extracranial mass arising in the adrenal medulla or sympathetic ganglia. Approximately half of all patients have high-risk features associated with a poor outcome - age >18 months, distant metastases and unfavorable histologic and genetic factors including amplification of the MYCN oncogene ${ }^{2}$. The success of anti-GD2 therapy that relies on immune cell-mediated cytotoxicity suggests that patients with neuroblastoma would benefit from other forms of immunotherapy; however, treatment results with use of cytotoxic CD8+ T lymphocytes directed against neuroblastoma antigens ${ }^{3}$, adoptive transfer of chimeric antigen receptor (CAR)-modified T cells ${ }^{4-6}$ or checkpoint inhibition ${ }^{7,8}$ have been suboptimal.

Major impediments to the effectiveness of immunotherapy in neuroblastoma are the immune evasion tactics deployed by the tumor cells as well as the tumor microenvironment $(\mathrm{TME})^{9}$. These include downregulation of major histocompatibility complex (MHC) class I molecules and defects in antigen-processing machinery (APM) that render neuroblastoma cells resistant to T-cellmediated cytotoxicity ${ }^{10-12}$, downregulation of cell-surface ligands required for natural killer (NK) cell receptor activation ${ }^{13}$, upregulation of checkpoint proteins that exert a protective role from NK cell-mediated lysis ${ }^{14}$, inefficient homing of cytotoxic T-cells to the tumor site $^{15}$ or tumor cell overexpression of the leukocyte surface antigen CD47, which enables avoidance of macrophagemediated phagocytosis ${ }^{16}$. Moreover, infiltration of suppressive immune cells such as $\mathrm{T}$ regulatory cells ${ }^{17}$, tumor-associated macrophages $(\mathrm{TAMs})^{18,19}$, myeloid-derived suppressor cells ${ }^{20}$ and secreted immunosuppressive factors such as TGF- $\beta$, contribute to the generation of a TME that hinders an effective immune response and further dampens the effects of adoptive cell therapies ${ }^{21}$. 
Sengupta et al.

Amplification of the MYCN oncogene poses another distinct challenge to immunotherapy in neuroblastoma. This transcription factor is amplified in approximately $50 \%$ of high-risk cases and is associated with aggressive disease and a poor clinical outcome $22,23 . M Y C N$-amplified tumors

77 consistently evade immune destruction by downregulating $\mathrm{MHC}$ class I molecules ${ }^{10}$ and are

78 associated with poor infiltration of cytotoxic $\mathrm{CD}^{+} \mathrm{T}$ cells ${ }^{24,25}$ and reduced expression of NK cell

79 ligands ${ }^{26}$. Interestingly, approximately half of high-risk neuroblastomas do not express amplified

$80 M Y C N$, and their capacity to induce a productive immune response remains unclear. In a recent

81 study that analyzed the immune gene expression programs associated with MYCN-nonamplified

82 tumors from high-risk patients, tumors with low as well as high functional tumor MYCN signatures

83 were observed to have significantly higher levels of NK and CD8+ T-cell infiltrates compared to

$84 \mathrm{MYCN}$-amplified tumors; although, somewhat counterintuitively, these findings translated into a

85 better outcome only in patients with high MYCN tumor signatures ${ }^{25,27}$.

Thus, although many of the mechanisms of immune evasion in neuroblastoma are known,

88 further understanding of the tumor-host interaction will be crucial to enhancing the ability of

89 immunotherapy to target and eliminate tumor-initiating and propagating cell populations.

90 Especially challenging is the genetic and biologic heterogeneity of this tumor which makes it

91 difficult to identify factors that consistently indicate the likelihood of an effective immune response

92 and hence identify patients who are most likely to benefit from this form of therapy. Thus, we

93 undertook an unbiased analysis of gene expression signatures across diverse clinical subtypes

94 of primary tumors and identify tumor cell state as an important predictor of immune 95 responsiveness in neuroblastoma. 
Sengupta et al.

\section{RESULTS}

\section{A subset of primary neuroblastomas express markers of a productive immune response}

To determine whether neuroblastomas are capable of eliciting a productive immune response, we first examined bulk RNA-sequencing data from 498 well-annotated primary human tumors representing diverse clinical and genetic subtypes (SEQC-498; GSE49711; Supplementary Fig.

1a) to quantify tumor-to-tumor gene expression variability and cluster tumor types based on gene expression profiles (see also Methods). In this unbiased analysis, all tumors within one cluster would share similar gene expression profiles, while being dissimilar to those of tumors within other clusters. Specifically, we first identified the top 5000 highly variably expressed genes within this dataset based on the premise that these would be most likely to contribute to distinct molecular subtypes $^{28,29}$ (Supplementary Fig. 1b; Supplementary Table 1). The data were dimensionally reduced using principal component analysis (PCA) and the top 20 leading principal components selected for clustering analysis (Supplementary Fig. 1c). Four distinct clusters were identified and visualized using 2D-Uniform Manifold Approximation and Projection (UMAP), a non-linear dimension-reduction too ${ }^{30,31}$ (Fig. 1a). To explore the transcriptional differences between the clusters, we identified the differentially expressed genes (DEGs) in each cluster and noted that tumors in cluster $1(\mathrm{C} 1 ; \mathrm{n}=103)$, termed Hi-MYCN, were enriched for MYCN target genes involved in cell proliferation and biosynthesis, and comprised $20 \%$ of the tumor set (Fig. 1a-c;

Supplementary Fig. 1d; Supplementary Table 2). Not surprisingly, this cluster segregated with MYCN-amplified tumors in patients aged $\geq 18$ months with stage 4 disease [according to the international neuroblastoma staging system (INSS)] $]^{32}$ and annotated "high risk" status (based on the Children's Oncology Group risk classification) ${ }^{33}$ (Fig. 1d; Supplementary Fig. 1e). The remaining clusters consisted of MYCN-nonamplified tumors (Fig. 1a) of which, cluster $2(\mathrm{C} 2, \mathrm{n}=$ 241), or neuronal, made up the largest proportion of tumors, $48 \%$, and comprised tumors that were enriched for DEGs with roles in nervous system 
bioRxiv preprint doi: https://doi.org/10.1101/2021.01.29.428154; this version posted January 30, 2021. The copyright holder for this preprint (which was not certified by peer review) is the author/funder. All rights reserved. No reuse allowed without permission.

Sengupta et al.

Fig. 1.

a

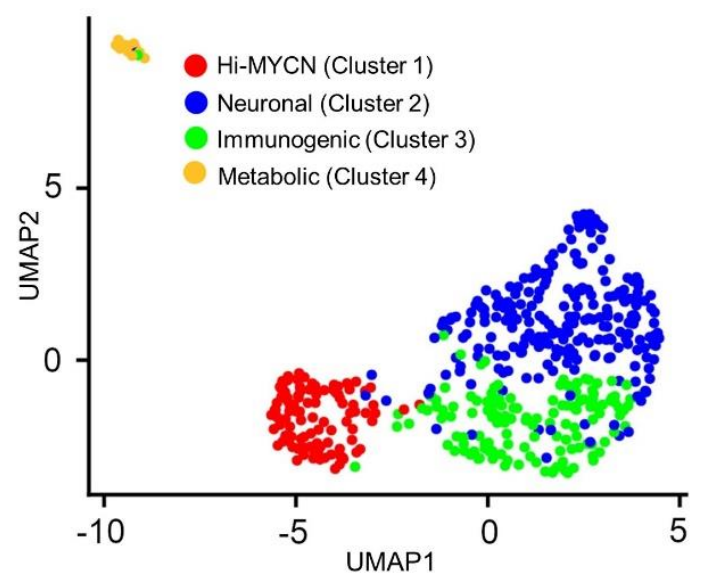

C

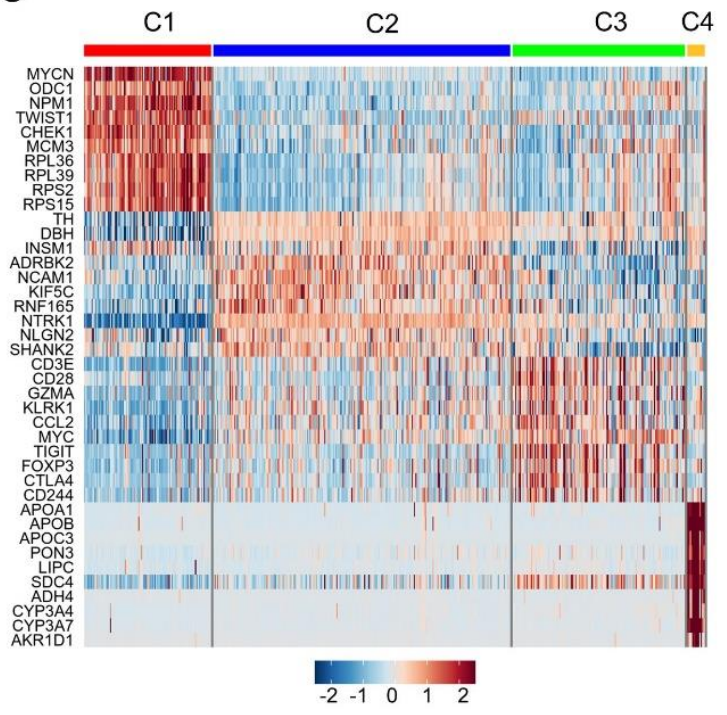

e

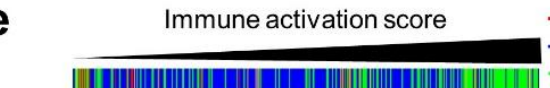

- Hi-MYCN

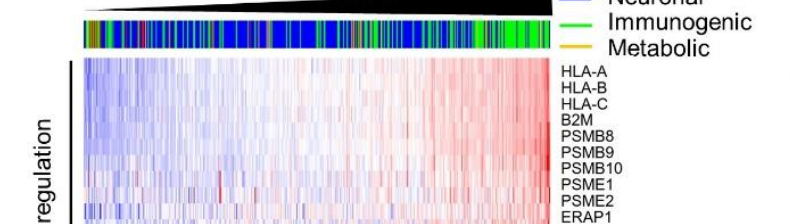

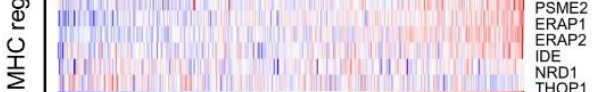

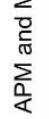

1
0

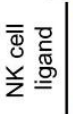

b
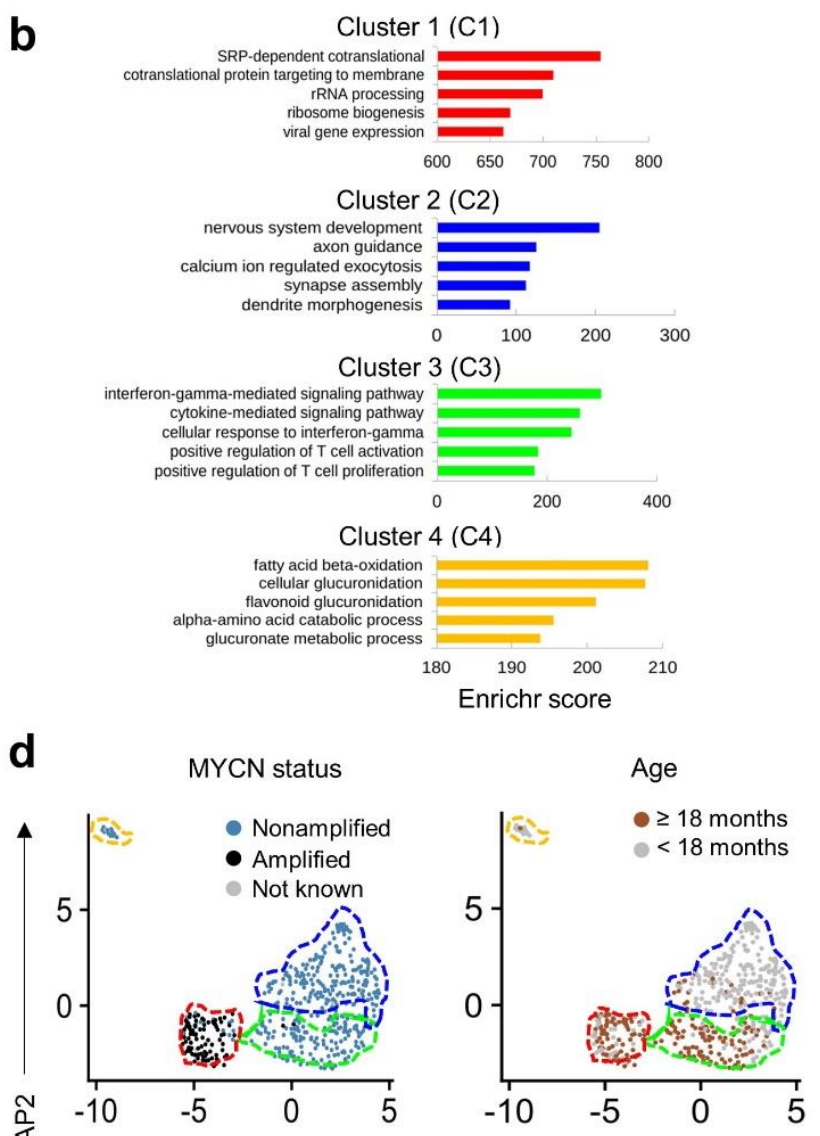

$\sum_{\supset}^{\nwarrow}$

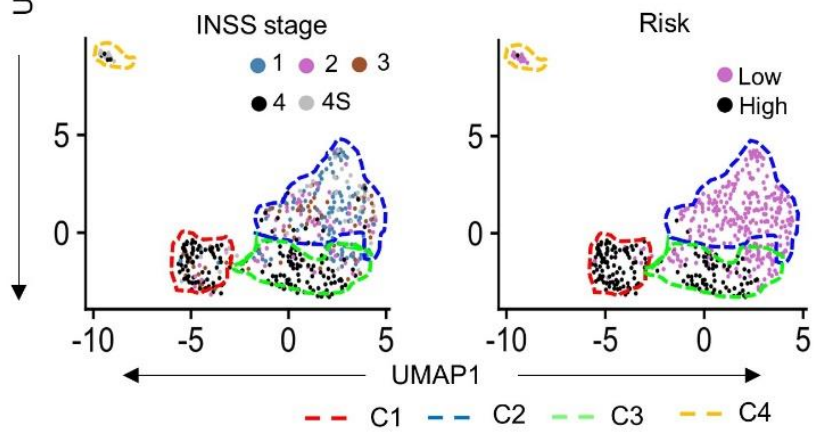

f

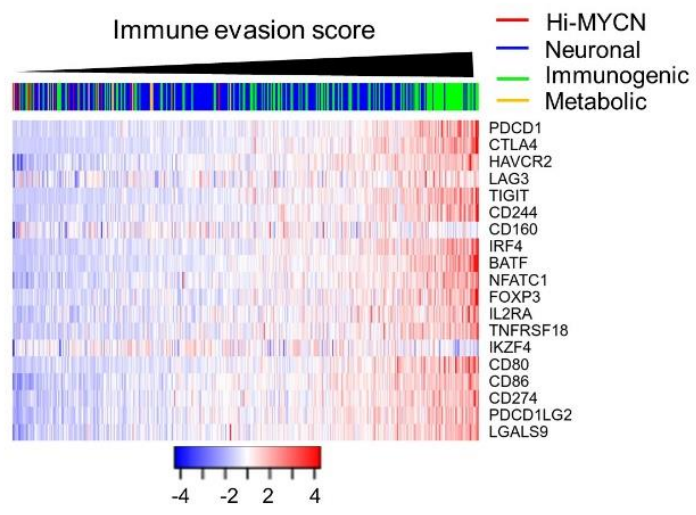


122 Fig. 1. A subset of neuroblastomas exhibits a productive immune response. (a) Two-

123 dimensional UMAP representations of the gene expression profiles in 498 neuroblastoma (NB)

124 tumors. Each dot represents a tumor. The top 5000 highly variable genes were selected based

125 on the variance-stabilizing method ${ }^{34}$ and the 20 significant principal components (PCs) selected

126 and processed in UMAP to generate four clusters representing four NB subtypes. The DEGs were

127 identified for each cluster using the receiver operating characteristics (ROC) curve to compare

128 one cluster with other three $\left(\log _{2} F C>0.25\right)$. (b) Gene ontology $(G O)$ analysis of top DEGs in the

129 four clusters. (c) Heat map of expression values of 10 representative DEGs within each cluster.

130 Rows are z-score scaled average expression levels for each gene in all four clusters. (d) UMAP

131 visualization of the distribution of the indicated prognostic features in NB among the four different

132 clusters. (e, f) Heat map of z-score transformed $\log _{2}$ normalized expression values of immune

133 activation (e) and evasion (f) genes in MYCN-nonamplified NBs ( $n=401)$. Tumors were ranked

134 based on increasing immune activation or evasion scores. Cluster annotations of the tumors are

135 indicated on the top horizontal bar. 
bioRxiv preprint doi: https://doi.org/10.1101/2021.01.29.428154; this version posted January 30, 2021. The copyright holder for this preprint (which was not certified by peer review) is the author/funder. All rights reserved. No reuse allowed without permission.

Sengupta et al.

\section{Supplementary Fig. 1.}

a

\begin{tabular}{|c|c|c|c|c|c|c|c|c|c|c|c|}
\hline \multirow{3}{*}{ 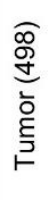 } & \multicolumn{2}{|c|}{$\begin{array}{c}\text { MYCN } \\
(\%)\end{array}$} & \multicolumn{2}{|c|}{$\begin{array}{l}\text { Age } \\
(\%)\end{array}$} & \multicolumn{2}{|c|}{$\begin{array}{l}\text { Risk } \\
(\%)\end{array}$} & \multicolumn{5}{|c|}{$\begin{array}{c}\text { INSS stage } \\
(\%)\end{array}$} \\
\hline & Nonamp & Amp & $<18 \mathrm{mo}$. & $\geq 18 \mathrm{mo}$. & Low & High & 1 & 2 & 3 & 4 & $4 \mathrm{~S}$ \\
\hline & $\begin{array}{c}401 \\
(80.5)\end{array}$ & $\begin{array}{c}92 \\
(18.4)\end{array}$ & $\begin{array}{c}300 \\
(60.3)\end{array}$ & $\begin{array}{c}198 \\
(39.7)\end{array}$ & $\begin{array}{c}322 \\
(64.6)\end{array}$ & $\begin{array}{c}176 \\
(35.4)\end{array}$ & $\begin{array}{c}121 \\
(24.3)\end{array}$ & $\begin{array}{c}78 \\
(15.7)\end{array}$ & $\begin{array}{c}63 \\
(12.6)\end{array}$ & $\begin{array}{c}183 \\
(36.7)\end{array}$ & $\begin{array}{c}53 \\
(10.6)\end{array}$ \\
\hline
\end{tabular}

b

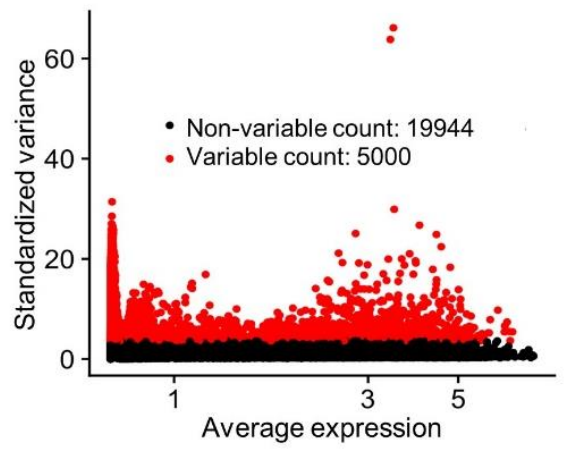

d

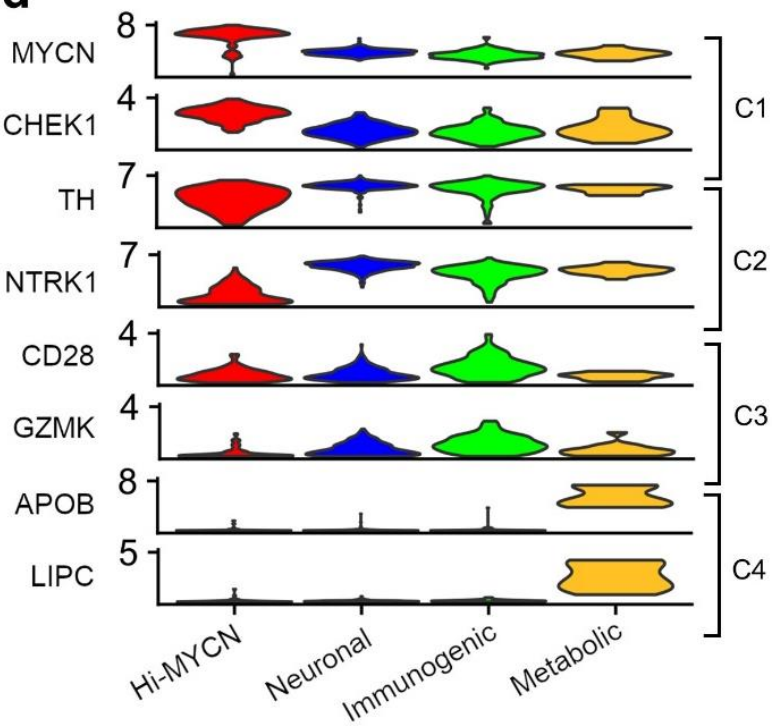

f

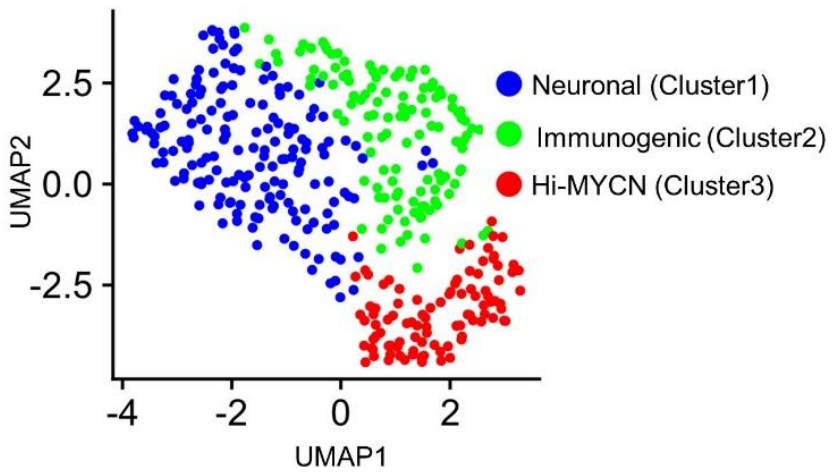

C

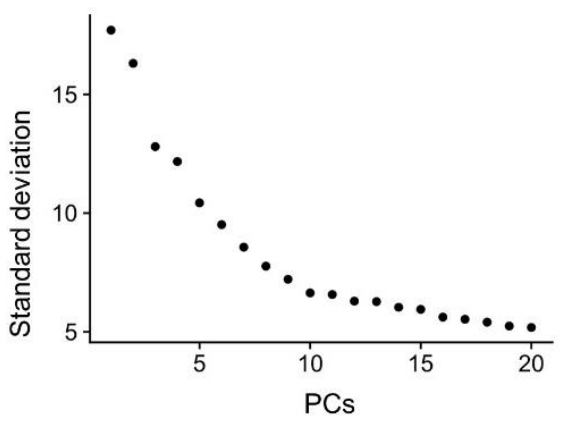

e

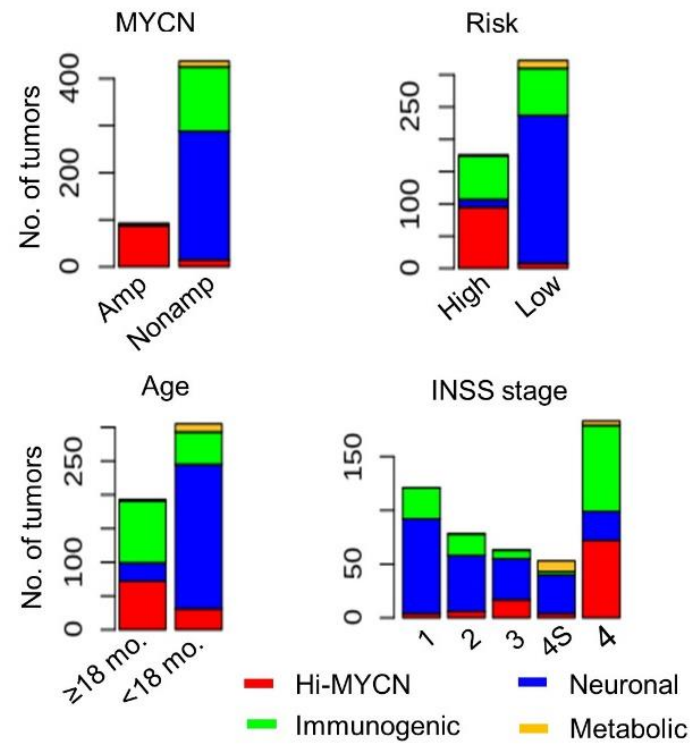

g

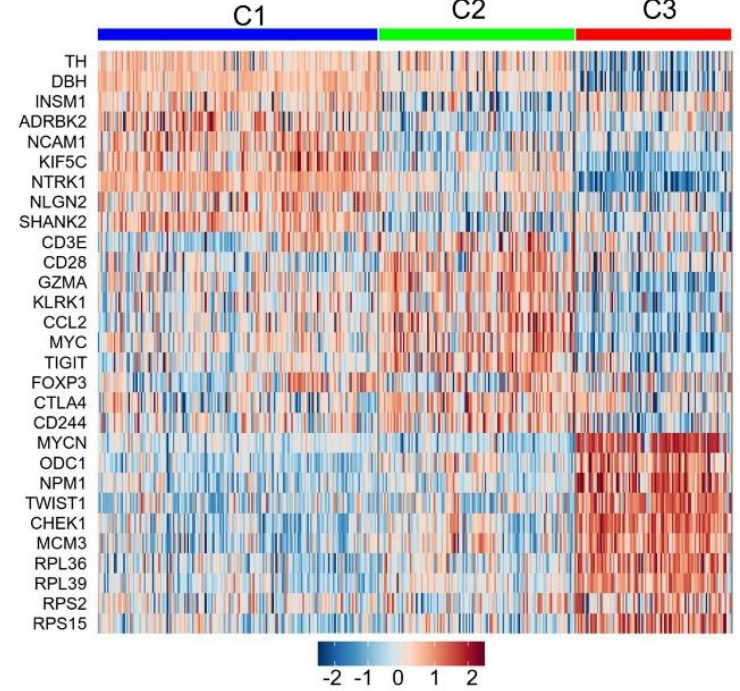


Sengupta et al.

136 Supplementary Fig. 1. Tumors within the immunogenic cluster express both immune

137 activation and evasion markers. (a) Distribution of the 498 primary NB tumors in the data set

138 (SEQC-498; GSE49711) within the indicated prognostic categories. (b) Scatter plot of the

139 standardized variance in expression of all protein coding genes within 498 tumors. Red dots

140 indicate the top 5000 variably expressed genes. (c) Elbow plot representing the percentage

141 variance for the top 20 principal components, PCs. (d) Violin plots showing the expression of

142 representative marker genes across the four clusters. (e) Stacked bar plots showing the

143 distribution of tumors within the defined prognostic features within each cluster. Amp, amplified;

144 Nonamp, nonamplified. (f) Two-dimensional UMAP representations of the gene expression

145 profiles in 394 NB tumors (GSE120572). Each dot represents a tumor. The top 3000 highly

146 variable genes were selected based on the variance-stabilizing method ${ }^{34}$ and the 20 significant

147 principal components (PCs) selected and processed in UMAP to generate three clusters

148 representing three NB subtypes. The DEGs were identified for each cluster using the receiver

149 operating characteristics $(\mathrm{ROC})$ curve to compare one cluster with other two $\left(\log _{2} \mathrm{FC}>0.25\right)$. (g)

150 Heat map of expression values of 10 representative DEGs within each cluster. Rows are z-score

151 scaled average expression levels for each gene in all three clusters. 
Sengupta et al.

\section{Supplementary Fig. 2.}

a

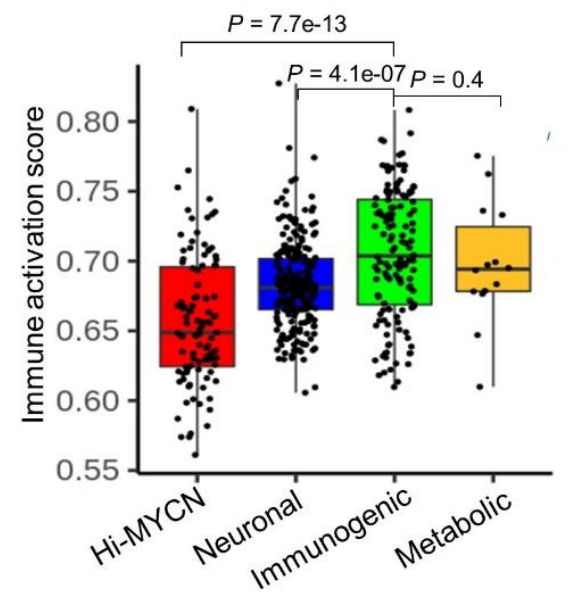

C

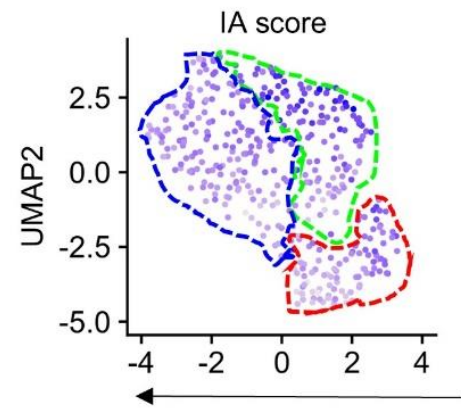

b

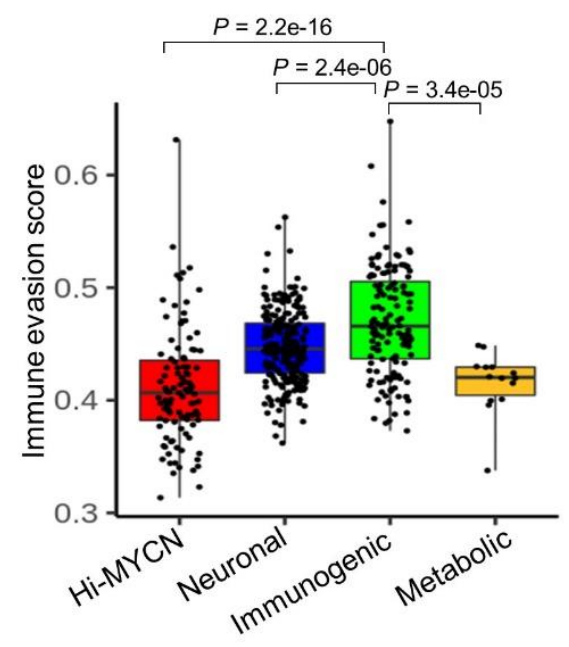

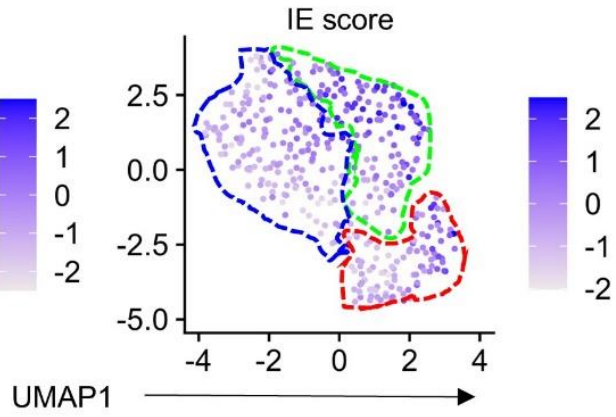


153 Supplementary Fig. 2. The immunogenic tumors are associated with markers of both

154 immune activation and evasion. (a, b) Box plots comparing immune activation (a) and evasion

155 (b) scores within the four clusters from the SEQC-498 tumor data set. All box plots are defined

156 by center lines (medians), box limits $\left(25^{\text {th }}\right.$ and $75^{\text {th }}$ percentiles), whiskers (minima and maxima;

157 the smallest and largest data range). Significance was determined by the Wilcoxon rank-sum test.

158 (c) UMAP visualization of the distribution of IA and IE scores among the three tumor clusters

159 derived from the 394 NBs in the GSE120572 dataset. Color bar represents normalized z-scores.

160 Values $<2.5$ and $>2.5$ were set to -2.5 and +2.5 respectively, to reduce the effects of extreme

161 outliers. 
Sengupta et al.

163 development Fig. 1a-c; Supplementary Fig. 1d). Cluster 3 (C3, $n=140)$, accounting for $28 \%$

164 of the tumors, was enriched for tumors whose DEGs were involved in immune function, such as

165 interferon-gamma (IFN-Y) response and $\mathrm{T}$ cell inflammation and activation, and hence were

166 designated immunogenic (Fig. 1a-c; Supplementary Fig. 1d) . Cluster 4 (C4; n=14; 3\%) was

167 clearly distinct from the other three clusters, and largely consisted of the spontaneously

168 regressing stage 4S tumors that were predominantly enriched for genes involved in fatty acid and

169 cholesterol homeostasis and hence were termed metabolic (Fig. 1a-c; Supplementary Fig. 1d).

170 The neuronal and metabolic tumors arose predominantly in children $<18$ months of age, were of

171 stages 1-3 and 4S, while the tumors within the immunogenic cluster were associated with patient

172 age $\geq 18$ months and metastatic disease ( $n=66 ; 47 \%)$ (Fig. 1d; Supplementary Fig. 1e). Thus,

173 our DEG-based analysis of almost 500 tumors categorized neuroblastoma into four largely distinct

174 groups that included a distinct subset, accounting for approximately one-third of the entire cohort,

175 whose gene expression profiles were closely linked to immune responsiveness. To ensure that

176 these results were not confined to one data set, we analyzed an independent data set of 394

177 tumors (GSE120572) using similar clustering methods. This cohort also segregated into Hi-

178 MYCN, neuronal and immunogenic clusters, again denoting the presence of immune response

179 gene expression in a subset of primary neuroblastomas, the majority of which lack MYCN

180 amplification (Supplementary Fig. 1f, g).

To pursue the immune genes that were differentially enriched in the immunogenic cluster, we

183 generated an immune activation (IA) score based on the relative expression of a curated set of

18441 genes known to have major roles in tumor cell-intrinsic immune functions, such as regulation

185 of $\mathrm{MHC}$ expression, antigen processing and presentation, NK cell recognition and T and NK cell

186 infiltration (Supplementary Table 3). After assigning an IA score to each of the MYCN-

187 nonamplified tumors $(n=401)$ in the SEQC-498 data set and arranging them in ascending order

188 (Supplementary Table 4), we observed that a significant number with the highest IA scores 
Sengupta et al.

189

190

191

192

193

194

195

196

197

198

199

200

201

202

203

204

205

206

207

208

209

210

211

212

213

214

predominantly fell within the immunogenic and metabolic clusters (Fig. 1e; Supplementary Fig.

2a), while those with intermediate or lower scores were associated with the neuronal and HiMYCN clusters, respectively (Fig. 1e; Supplementary Fig. 2a). Because a cytotoxic immune response is generally accompanied by immune suppression or evasion ${ }^{35,36}$, we determined whether immune suppression was also represented in the $M Y C N$-nonamplified tumors by ranking them in ascending order of an immune evasion (IE) score based on the relative expression of 19 genes, most of which were markers of T-cell dysfunction (Supplementary Table 3). Again, the immunogenic tumor cluster had significantly higher IE scores compared with the neuronal and metabolic clusters (Fig. 1f; Supplementary Fig. 2b). Moreover, we observed enrichment for IA and IE scores in the immunogenic cluster in the additional data set (GSE120572) (Supplementary Fig. 2c), thus strengthening our premise that these tumors maybe capable of eliciting an immune response.

Consistent with the known poor immunogenicity of $M Y C N$-amplified tumors ${ }^{24,25}$, we also observed that tumors within the Hi-MYCN cluster had, on the whole, the lowest IA and IE scores (Supplementary Fig. 2a, b). Surprisingly, however, a small subset within this cluster had scores that were comparable to the highly immunogenic tumors within the immunogenic cluster [13 of $103(12.6 \%)$ above the median for immunogenic tumors] (Supplementary Fig. 2a, b). Thus, while the majority of neuroblastomas do not possess an immune response gene signature, a subset has significantly increased expression of both immune activation and evasion markers, pointing to their ability to induce an anti-tumor immune response.

The mesenchymal lineage is preferentially associated with immune response signatures in neuroblastoma

Having identified subsets of neuroblastomas with the potential for immunogenicity, we next sought a biomarker that might consolidate the complex interactions between the immune system and the 
Sengupta et al.

215 tumor. To this end, we performed a modular gene co-expression analysis of the 140

216 transcriptomes within the immunogenic cluster in the SEQC-498 data set to identify biologically

217 relevant pathways based on similar gene expression patterns. Using the CEMiTool (co-

218 expression modules identification tool) package (Russo et al., 2018), we identified five gene co-

219 expression modules (M1-M5) within the immunological cluster (Supplementary Fig. 3a). Among

220 these modules, M1, with the highest number of co-expressed genes, contained gene sets

221 enriched for epithelial to mesenchymal transition (EMT), inflammatory response, and interferon

222 signaling, suggesting an association between EMT and the preponderant representation of

223 immune marker genes within the immunogenic cluster (Fig. 2a; Supplementary Fig. 3b).

224 Furthermore, integration of the co-expression data in module M1 with protein-protein interaction

225 data from the STRING 11.0 database identified mesenchymal lineage and immune markers as

226 top regulatory hubs (Fig. 2b), leading us to hypothesize that in neuroblastoma, tumor

227 immunogenicity could be determined by cell state.

229 Two independent groups ${ }^{37,38}$ recently described two distinct cell states in neuroblastoma: a 230 differentiated sympathetic neuron-like adrenergic (ADR) phenotype, defined by lineage markers 231 including $P H O X 2 B, D B H$, and $T H$, and a mesenchymal (MES) phenotype, characterized as

232 "neural crest cell-like" (NCC), and expressing genes such as PRRX1, FOSL1, and FOSL2. To 233 test our prediction, we first quantified the adrenergic and mesenchymal identities of each tumor

234 in our cohort based on the expression levels of the lineage-specific genes in each cell state as 235 established by Groningen et $\mathrm{al}^{38}$ (see Methods). We ensured that there was no overlap between 236 the 369-gene adrenergic signature and the genes that made up the IA data set and removed the

2376 IA genes that were also present in the 485-gene mesenchymal signature. Next, we assigned 238 either an adrenergic (A-score) or a mesenchymal (M-score) score to each tumor within our four 239 previously identified clusters. This analysis revealed significant enrichment of the mesenchymal 240 cell state within the immunogenic and metabolic clusters (Fig. 2c; Supplementary Fig. 3c). 
bioRxiv preprint doi: https://doi.org/10.1101/2021.01.29.428154; this version posted January 30, 2021. The copyright holder for this preprint (which was not certified by peer review) is the author/funder. All rights reserved. No reuse allowed without permission.

Sengupta et al.

Fig. 2.
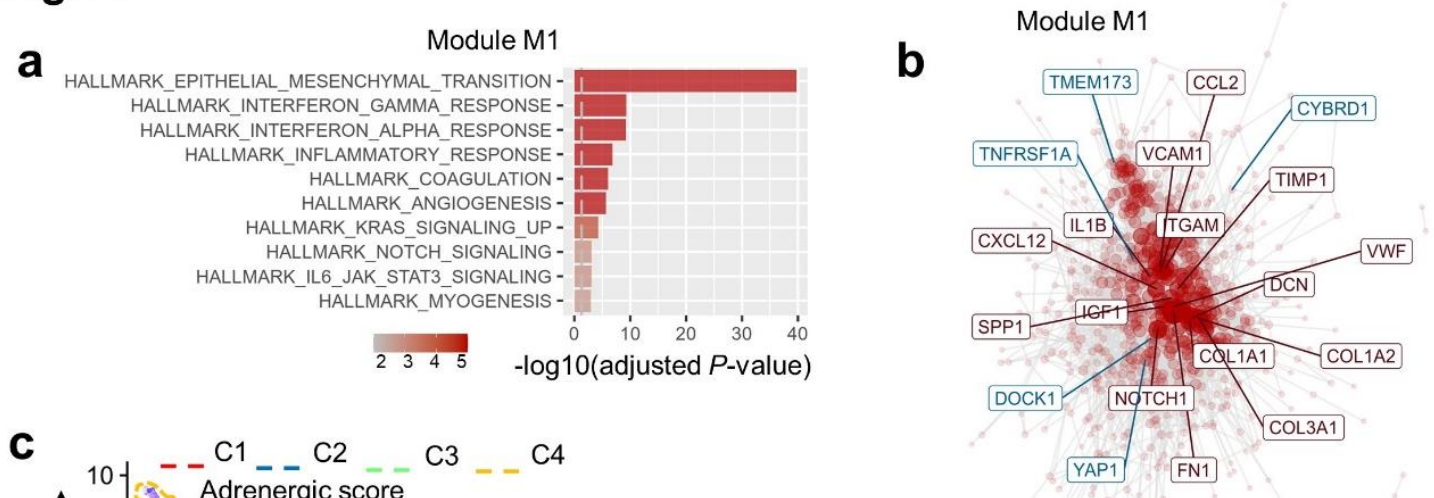

$\underline{\text { Hub }}$

CO-EXPRESSION INTERACTION

Degree

50

100

150

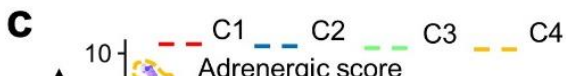

d

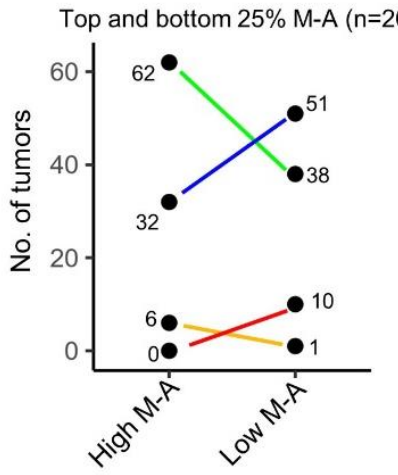

f

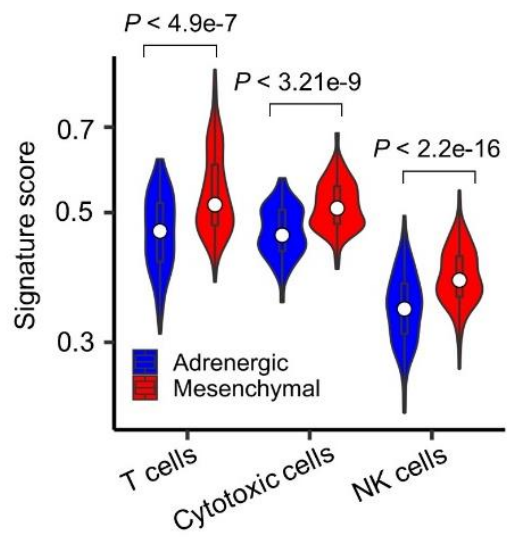

g

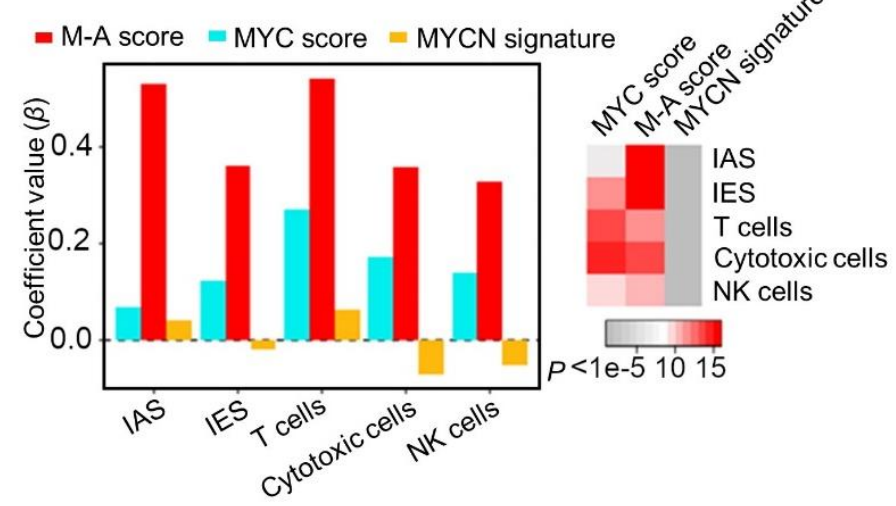

C4

e

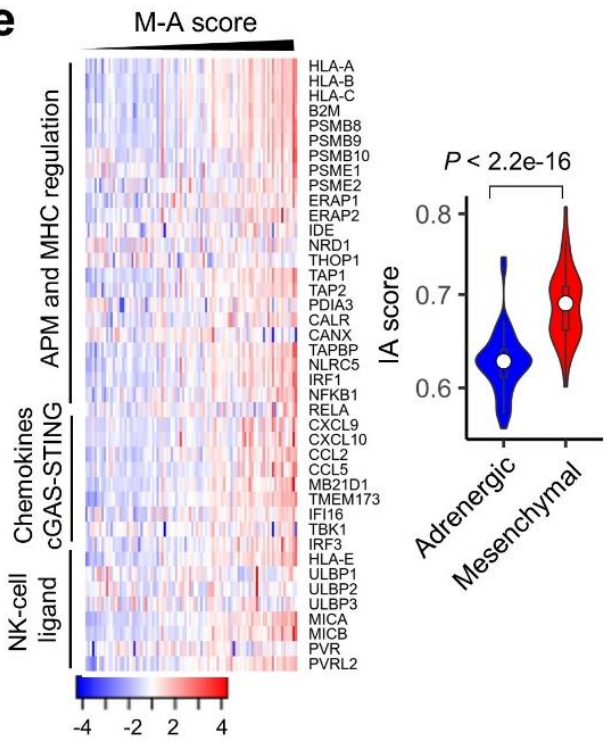

h

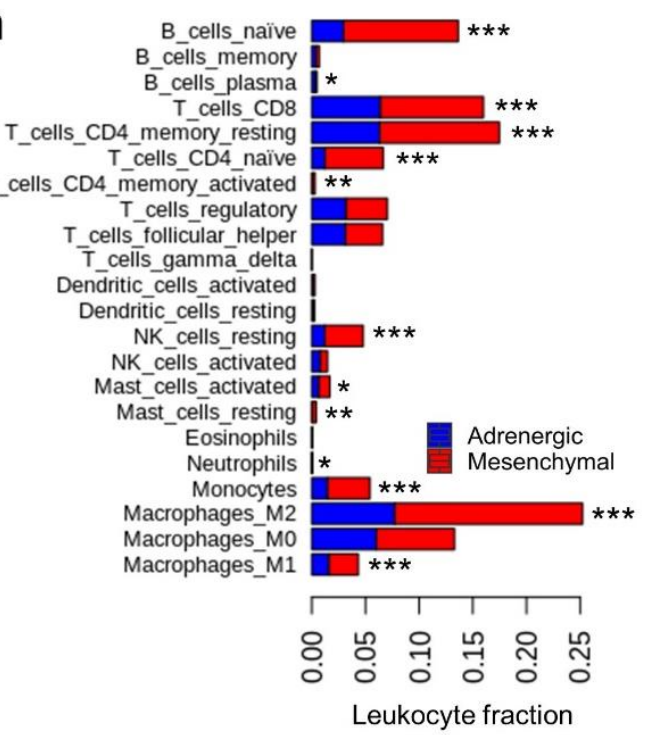


Sengupta et al.

241

242

243

244

245

246

247

248

249

250

251

252

253

254

255

256

257

258

259

260

261

262

263

264

265

266

Fig. 2. The mesenchymal cell state is associated with an immunogenic signature in NB. (a) GO analysis of co-expressed genes associated with module M1 using the KEGG (Kyoto encyclopedia of genes and genomes) database. The vertical dashed line indicates the adjusted $P$-value of 0.05 . (b) Gene network representing all possible interactions in module M1. The topmost connected genes (hubs) are indicated. Hubs derived from module M1 are colored blue (co-expression) and those from the STRING database are indicated in red (interaction). The size of each node corresponds to the degree of interaction. (c) UMAP visualization of the distribution of adrenergic (top) and mesenchymal scores (bottom) among the four tumor clusters. Color bar represents normalized z-scores. Values $<2.5$ and $>2.5$ were set to -2.5 and +2.5 respectively, to reduce the effects of extreme outliers. (d) Dot plots showing the distribution of $M Y C N$ nonamplified tumors $(n=400)$ within each of the clusters based on ranked M-A scores. Left, Tumors from the upper (high M-A) and lower (low M-A) M-A score quartiles are shown $(n=200$; $P<0.01$ for C3). Right, Representations based on the median M-A scores of the entire tumor cohort ( $\mathrm{n}=400 ; P=0.05$ for C3). Fisher's exact test was used for both calculations. (e) Left, Heatmap representation of the expression of tumor cell-intrinsic immune activation genes in MYCN-amplified tumors $(n=92)$. Samples are ranked by increasing $M-A$ score. $\log _{2}$ gene expression values were z-score transformed for heatmap visualization. Right, Violin plots of the distribution of immune activation scores in the tumors on the left, classified either as adrenergic or mesenchymal, based on the median M-A score. The box plots within the violin plots are defined by center lines (medians), box limits $\left(25^{\text {th }}\right.$ and $75^{\text {th }}$ percentiles), whiskers (minima and maxima; $1.5 \mathrm{X}$ the interquartile range). Significance was determined by the two-sided Kolmogorov-Smirnov (KS) test. APM, antigen processing machinery. (f) Violin plots comparing the quantitative scores of the indicated immune cell signatures in 100 tumors from the upper (mesenchymal) and lower (adrenergic) quartiles of the tumor M-A scores using the two-sided KS test. The box plots within the violin plots are defined as in (D). (g) Left, Bar diagram comparing regression coefficient $(\beta)$ values derived from multivariate multiple regression model analysis of $M Y C N$-nonamplified 
267 tumors. $\beta$-coefficient values were compared between three predictors: MYC score, M-A score and

268 MYCN signature. IA, IE, T cell, cytotoxic cell and NK cell scores were used as response variables

269 to generate the model ${ }^{39}$. Right, Heat map of the $P$-values associated with the three predictors. (h)

270 Bar diagram comparing the CIBERSORT-estimated fractional content of the indicated tumor-

271 infiltrating leukocytes between $M Y C N$-nonamplified adrenergic and mesenchymal tumors.

272 Adrenergic and mesenchymal tumors were assigned as in (f). Data represent the means, $n=100$

273 tumors, ${ }^{*} P<0.05,{ }^{* *} P<0.01,{ }^{* * *} P<0.001$ two-tailed Welch's t-test. 
bioRxiv preprint doi: https://doi.org/10.1101/2021.01.29.428154; this version posted January 30, 2021. The copyright holder for this preprint (which was not certified by peer review) is the author/funder. All rights reserved. No reuse allowed without permission.

Sengupta et al.

\section{Supplementary Fig. 3.}
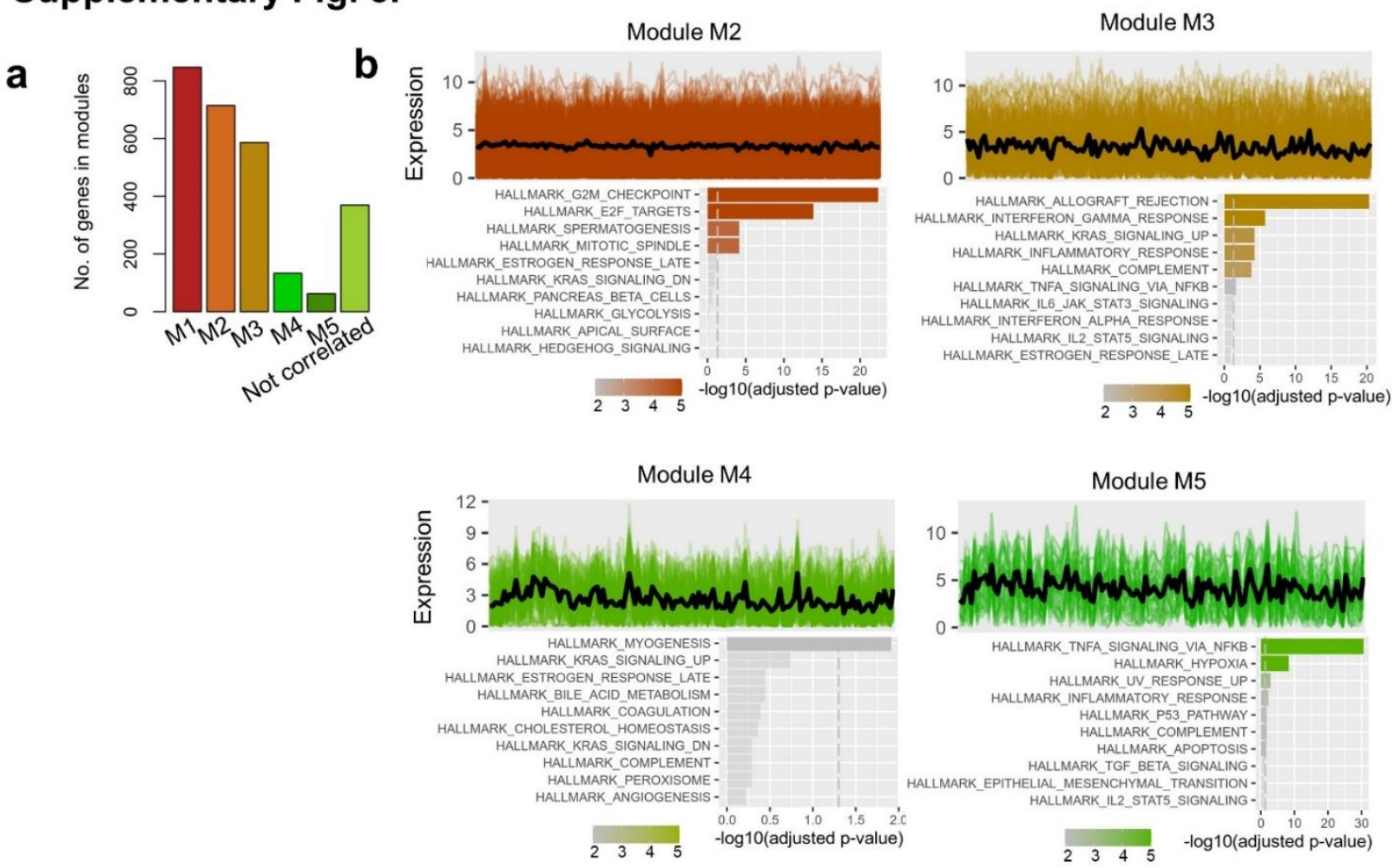

C

\begin{tabular}{|c|c|c|c|c|}
\hline $\begin{array}{c}\text { Lineage }(\mathrm{no} \\
\text { of genes })\end{array}$ & $\begin{array}{c}\text { Hi-MYCN } \\
(2859)(P \text {-value })\end{array}$ & $\begin{array}{c}\text { Neuronal } \\
(1416)(P \text {-value })\end{array}$ & $\begin{array}{c}\text { Immunogenic } \\
(2213)(P \text {-value })\end{array}$ & $\begin{array}{c}\text { Metabolic } \\
(2043)(P \text {-value })\end{array}$ \\
\hline $\begin{array}{c}\text { Adrenergic } \\
(369)\end{array}$ & $121(<0.001)$ & $78(<0.001)$ & 16 (n.s.) & 13 (n.s.) \\
\hline $\begin{array}{c}\text { Mesenchymal } \\
(479)\end{array}$ & 46 (n.s.) & 31 (n.s.) & $129(<0.001)$ & $156(<0.001)$ \\
\hline
\end{tabular}

d

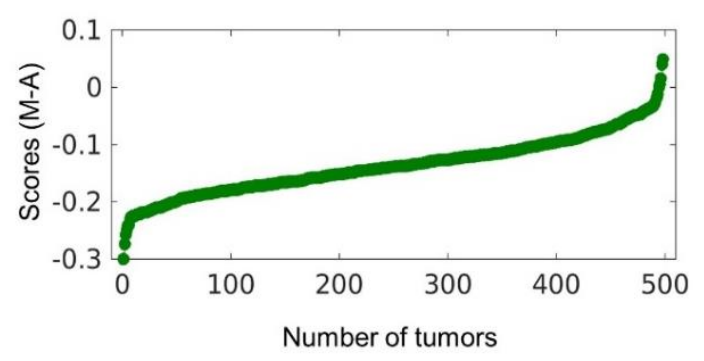

f

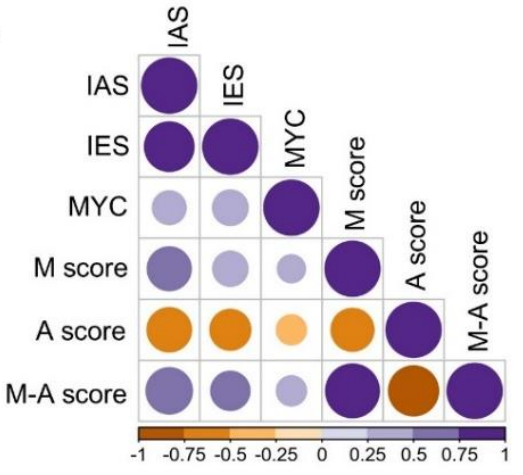

e

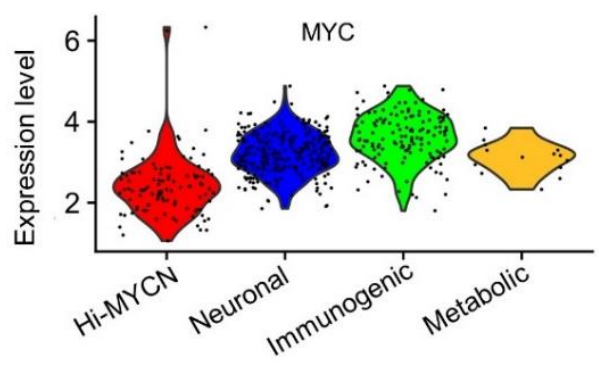

g

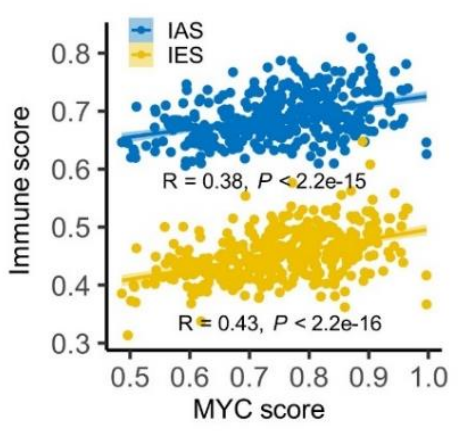


Sengupta et al.

274 Supplementary Fig. 3. Cell lineage markers are significantly associated with immune gene

275 signatures in NB. (a) Bar plots representing the numbers of co-expressed genes within each

276 module. (b) Profile plots depicting the expression levels ( $y$-axis) of individual genes (colored lines)

277 and their mean expression (black line) in 140 tumors (x-axis) from modules M2, M3, M4 and M5.

278 GO analysis of co-expressed genes associated with each module using the KEGG database is

279 appended below the respective profile plot. The vertical dashed line indicates the adjusted $P$ -

280 value of 0.05 . (c) Summary of the overlap between the DEGs associated with the four tumor

281 clusters and the adrenergic or mesenchymal signature genes as per Groningen et al., 2017.

282 Significance was determined by Fisher's exact test. (d) Scatter plot of the 498 primary NB tumors

283 ranked based on increasing M-A score. (e) Violin plots of the distribution of normalized expression

284 levels of MYC in the four tumor clusters. (f) Pearson correlation matrix showing pairwise

285 correlation values among the indicated parameters. The colors and sizes of the circles indicate

286 the correlation coefficient values, with the least (smaller, orange circles) to the most (larger, blue

287 circles) degree of association between the parameters shown. (g) Scatter plot of the correlation

288 between MYC expression and immune activation (blue dots) or evasion (yellow dots) scores in

289 MYCN-nonamplified tumors. 
bioRxiv preprint doi: https://doi.org/10.1101/2021.01.29.428154; this version posted January 30, 2021. The copyright holder for this preprint (which was not certified by peer review) is the author/funder. All rights reserved. No reuse allowed without permission.

Sengupta et al.

\section{Supplementary Fig. 4.}

a
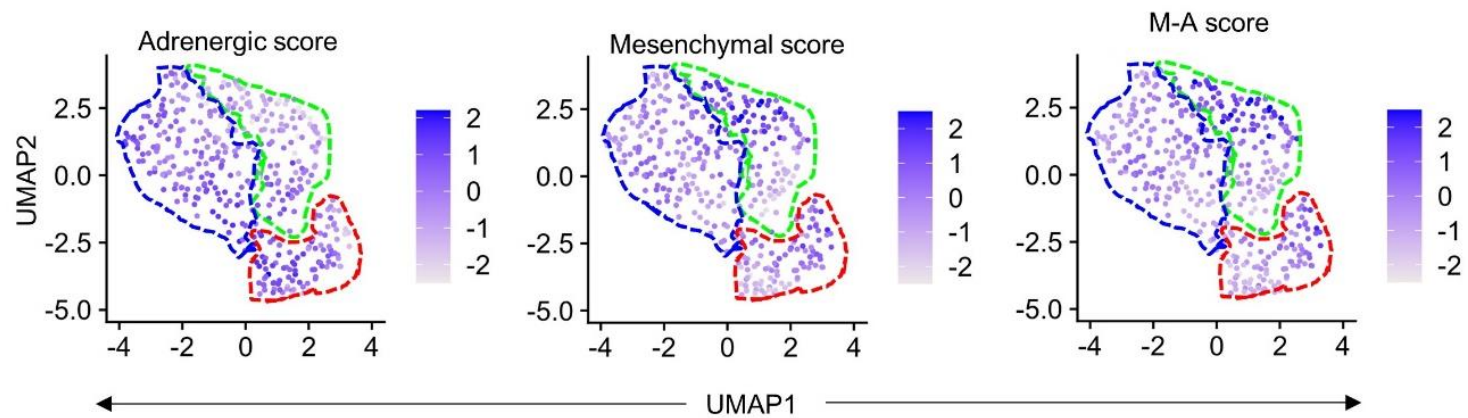

b

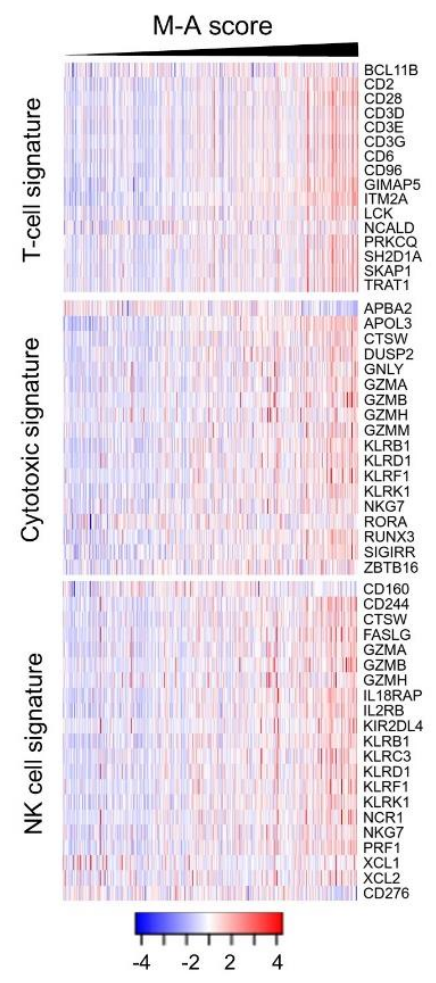

e

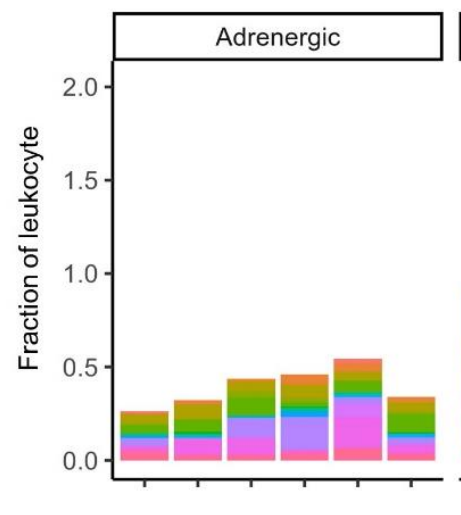

C

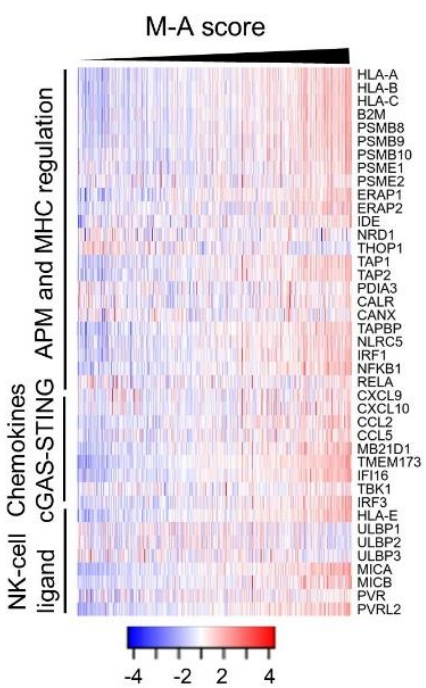

d
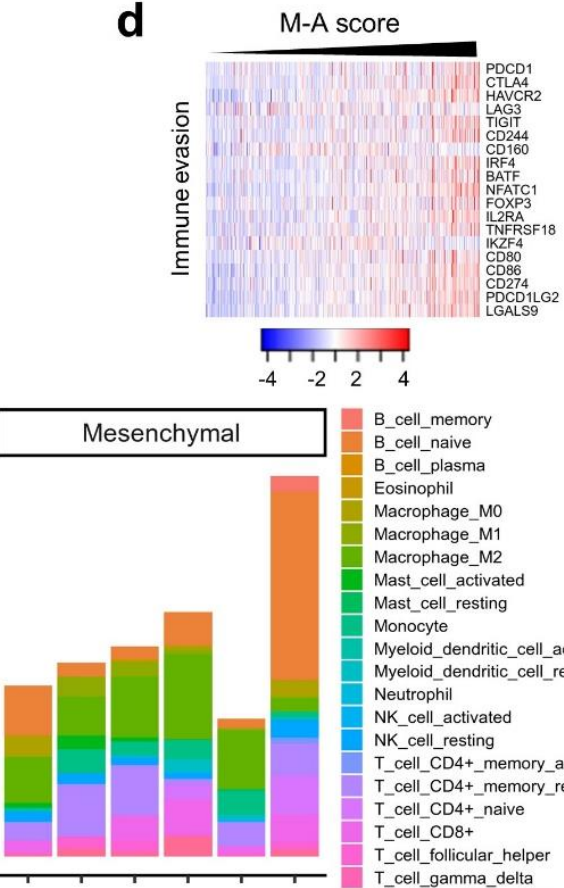

B_cell_memory B_cell_naive B_cell_plasm

Eosinophil

Macrophage_M1

Macrophage_M2

Mast_cell_activate

Mast_cell_resting

Monocyte

Myeloid_dendritic_cell_activated

Myeloid_dendritic_cell_resting

Neutrophil

NK_cell_activated

T_cell_CD4+_memory_activated

T_cell_CD4+_memory_resting

T_cell_CD4+_naive

T_cell_CD8+

T_cell_follicular_helper

T_cell_gamma_delta

T_cell_regulatory_(Tregs)
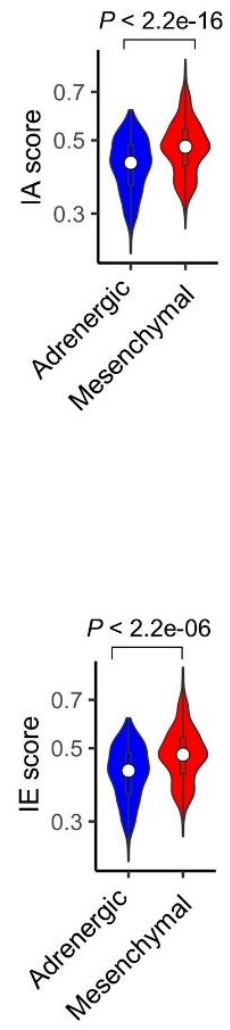
Sengupta et al.

291 Supplementary Fig. 4. The relative mesenchymal score (M-A score) is positively correlated

292 with an immunogenic signature. (a) UMAP visualization of the distribution of adrenergic,

293 mesenchymal, and M-A scores among the three tumor clusters derived from 394 NBs in the

294 GSE120572 dataset. Color bar represents normalized z-scores. Values <2.5 and >2.5 were set

295 to -2.5 and +2.5 respectively, to reduce the effects of extreme outliers. (b) Heat maps of the

296 indicated immune cell signatures in MYCN-nonamplified tumors, ranked by increasing M-A

297 scores. Log $_{2}$ gene expression values were z-score transformed for heatmap visualization. (c, d)

298 Heatmaps depicting the immune activation (c) and evasion (d) signatures in MYCN-nonamplified

299 tumors, ranked by increasing M-A score. Log 2 gene expression values were $\mathrm{z}$-score transformed

300 for visualization. Violin plots comparing the distribution of immune activation (c) and evasion (d)

301 signatures in 100 tumors from upper (mesenchymal) and lower (adrenergic) quartiles of the M-A

302 score are shown next to the heatmaps. Significance determined by the two-sided KS test. Box

303 plots within the violin plots are defined by center lines (medians), box limits $\left(25^{\text {th }}\right.$ and $75^{\text {th }}$

304 percentiles), whiskers (minima and maxima; 1.5X the interquartile range). (e) Bar graph

305 comparing the CIBERSORT-estimated fractional content of the indicated tumor-infiltrating

306 leukocytes in six tumors with the uppermost (mesenchymal) and lowermost (adrenergic) M-A

307 scores. 
Sengupta et al.

308

By contrast, the Hi-MYCN and neuronal clusters were enriched for the adrenergic cell state (Fig.

\section{2c; Supplementary Fig. 3c).}

Next, to identify predictors of immunogenicity among the $M Y C N$-nonamplified tumors, we calculated the relative mesenchymal score for each tumor by subtracting the adrenergic from the mesenchymal score (M-A score). This resulted in a continuum of low to high M-A scores, corresponding to a less mesenchymal to a more mesenchymal tumor state (Supplementary Fig.

3d). To determine whether these cell states had any effect on immune response in the MYCNnonamplified tumors, we determined whether the mesenchymal, adrenergic, or M-A scores correlated with our previously defined immune activation and evasion scores (Fig. 1e, f). We also tested the effect of $M Y C$, one of the top differentially expressed genes in the immunogenic cluster (Supplementary Fig. 3e). MYC is overexpressed in approximately $10 \%$ of $M Y C N$-nonamplified neuroblastomas ${ }^{40}$ and regulates the expression of cell-intrinsic immune evasion markers in lymphoma ${ }^{41}$. Of these variables, the M-A score showed the strongest correlation with immunogenicity, not only in terms of the immune activation score $(R=0.71)$, but also the immune evasion score $(R=0.51)$ (Supplementary Fig. 3f). MYC expression was only modestly correlated with immune evasion and activation scores $(R=0.43$; $R=0.38$, respectively) (Supplementary Fig. 3g). With no overlap between the lineage marker and immune response gene sets, these results suggest that the relative abundance of a mesenchymal signature (M-A score) is a better predictor of immune response than individual adrenergic or mesenchymal signatures. In agreement, tumors with high M-A scores were represented at a significantly higher proportion within the immunogenic cluster compared to tumors with low M-A scores (Fig. 2d), a result that was recapitulated in our second data set, (GSE120572) (Supplementary Fig. 4a). Finally, our finding of the subset of tumors marked by the relatively high expression of immune activation and evasion genes within the Hi-MYCN cluster (Supplementary Fig. 2a, b) prompted us to further evaluate the cell states of these tumors. Ranking these tumors based on increasing M-A scores revealed a positive relationship between cell-intrinsic immunogenicity and the mesenchymal state 
Sengupta et al.

334 (Fig. 2e), suggesting that similar to our results in MYCN-nonamplified tumors, the presence of

335 immune gene expression in $M Y C N$-amplified tumors is significantly correlated with the

336 mesenchymal phenotype.

The preferential overexpression in the mesenchymal phenotype of tumor cell-intrinsic genes that induce a positive immune response (IA score), as well as its correlation with transcripts that suppress the immune response (IE score), suggested that these tumors may support increased

341 immune cell infiltration. To test this prediction, we used two orthogonal approaches. First, we 342 assessed whether established signatures of immune cell infiltration ${ }^{39,42}$ were present in the 343 tumors arranged according to increasing M-A scores, and observed enrichment for signatures of 344 infiltrating immune cells in tumors with mesenchymal phenotypes (Supplementary Fig. 4b).

345 Intriguingly, we also noted that adrenergic tumors were enriched for CD276 (B7-H3) expression, 346 an immune checkpoint marker that protects neuroblastoma cells from NK cell-mediated 347 cytotoxicity, which may partly account for the decreased immune response signatures in these 348 cells $^{14}$ (Supplementary Fig. 4b). We next quantified the immune response signatures in a subset 349 of tumors $(n=100)$ from both the upper and lower quartiles of the M-A score that had significant 350 differences in their activation and evasion scores (Supplementary Fig. 4c, d). High M-A scoring 351 (mesenchymal) tumors had significantly higher expression levels of cytotoxic T and NK cell 352 signatures compared with those of low M-A scoring (adrenergic) tumors (Fig. 2f). Considering 353 that the M-A score and, to a lesser extent, higher MYC expression were positively associated with

354 IA and IE scores in pairwise testing (Supplementary Fig. 3f), we next assessed their relative 355 contributions as independent predictors of an immune response in a multivariate multiple 356 regression model consisting of immune activation and evasion scores and $\mathrm{T}$ and NK cell 357 signatures (Fig. 2g). Because MYCN amplification is linked to immune suppression, we also 358 included the 157-gene MYCN signature generated by Valentijn et al ${ }^{27}$ and subsequently used by 359 Wei et al to identify immune predictors in $M Y C N$-nonamplified neuroblastoma ${ }^{25}$. In this analysis 
Sengupta et al.

360

361

362

363

364

365

366

367

368

369

370

371

372

373

374

375

376

377

378 Tumor cell-intrinsic upregulation of immune pathways in mesenchymal neuroblastoma

379 We next sought to understand the extent to which the presence or absence of an immunogenic

380 signature in the bulk RNA-sequencing data was intrinsic to tumor cells or was conferred by the

381 tumor microenvironment. Analysis of the lineage identities of a panel of 24 human neuroblastoma

382 cell lines (15 MYCN-amplified; 9 MYCN-nonamplified) from RNA-sequencing data (GSE28019)

383 revealed a gradient of adrenergic-to-mesenchymal scores (Supplementary Fig. 5a). Consistent 
bioRxiv preprint doi: https://doi.org/10.1101/2021.01.29.428154; this version posted January 30, 2021. The copyright holder for this preprint (which was not certified by peer review) is the author/funder. All rights reserved. No reuse allowed without permission.

Sengupta et al.

Fig. 3.
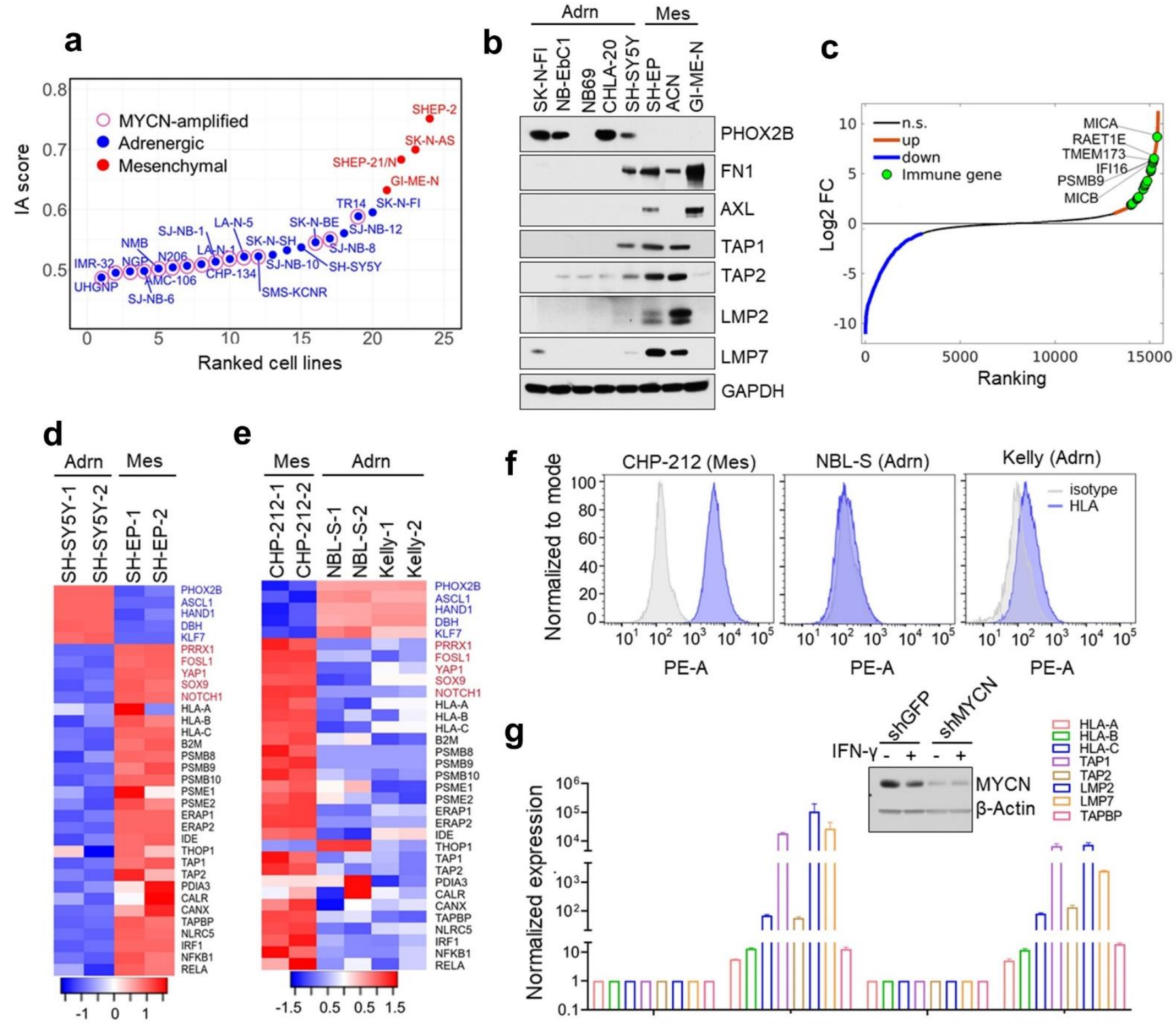

g

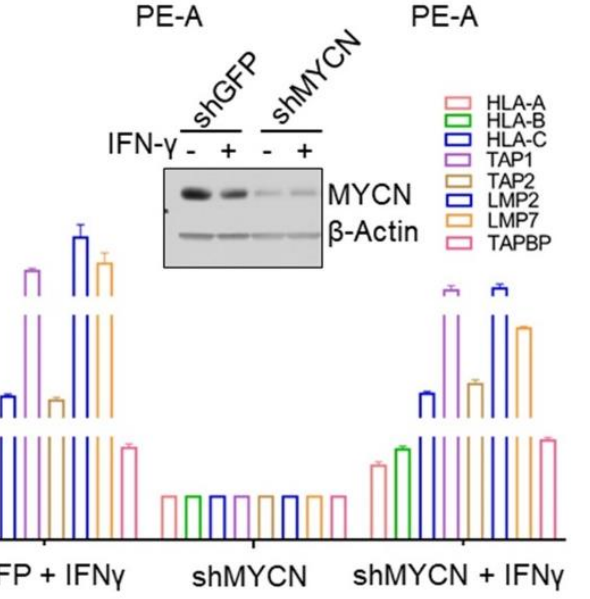


Sengupta et al.

Fig. 3. Tumor cell-intrinsic immune marker genes are upregulated in mesenchymal NBs.

(a) Scatter plot of the immune activation (IA) scores of human neuroblastoma cell lines (RNA-seq data; GSE28019). Cell lines are arranged based on increasing IA scores and designated as adrenergic or mesenchymal based on lineage-specific gene expression. (b) Western blot (WB) analysis of adrenergic (PHOX2B) and mesenchymal (FN1, AXL) cell lineage markers and antigen processing genes (TAP1/2, LMP2/7) in MYCN-nonamplified NB cell lines. GAPDH was used as the loading control. Adrn, adrenergic; Mes, mesenchymal. (c) Waterfall plot of the fold-change in

391 RNA expression levels of up- and downregulated genes in SH-EP compared to SH-SY5Y NB 392 cells; selected immune genes are highlighted in green. (d, e) Heat maps of lineage marker (blue, 393 adrenergic; red, mesenchymal) and MHC and antigen processing machinery gene (black) expression in the indicated MYCN-nonamplified (d) and MYCN-amplified (CHP-212, Kelly) and overexpressing (NBL-S) (e) adrenergic and mesenchymal cells ( $n=2$ biological replicates). Rows are z-scores calculated for each transcript in each cell type. (f) Fluorescence activated cell sorting

397 (FACS) analysis of cell surface HLA expression in the cells depicted in e. Isotype controls are depicted in gray. The X-axis denotes fluorescence intensity of indicated proteins using 399 phycoerythrin (PE-A) tagged antibodies. Results representative of 2 independent experiments.

400 (g) RT-qPCR analysis of antigen processing and presentation genes in MYCN-amplified Kelly NB 401 cells engineered to express shMYCN or shGFP (control) with or without IFN-y induction (100 $402 \mathrm{ng} / \mathrm{mL}$ for $24 \mathrm{hr}$.). Data are normalized to GAPDH and represent means $\pm \mathrm{SD}, n=2$ biological 403 replicates. Inset, WB analysis of MYCN in control and shMYCN cells. Actin was used as a loading 404 control. 
bioRxiv preprint doi: https://doi.org/10.1101/2021.01.29.428154; this version posted January 30, 2021. The copyright holder for this preprint (which was not certified by peer review) is the author/funder. All rights reserved. No reuse allowed without permission.

Sengupta et al.

\section{Supplementary Fig. 5.}

a

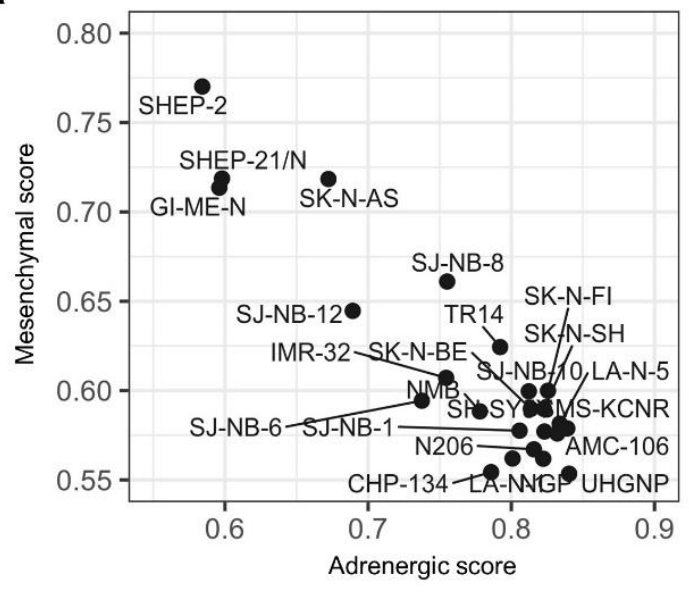

C
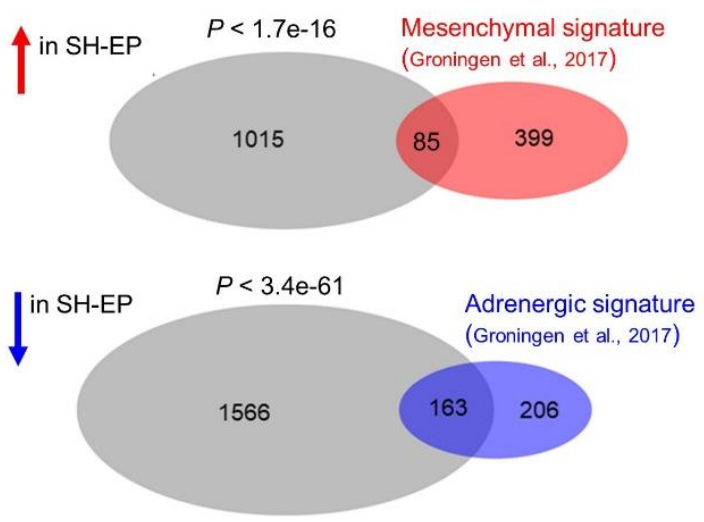

d

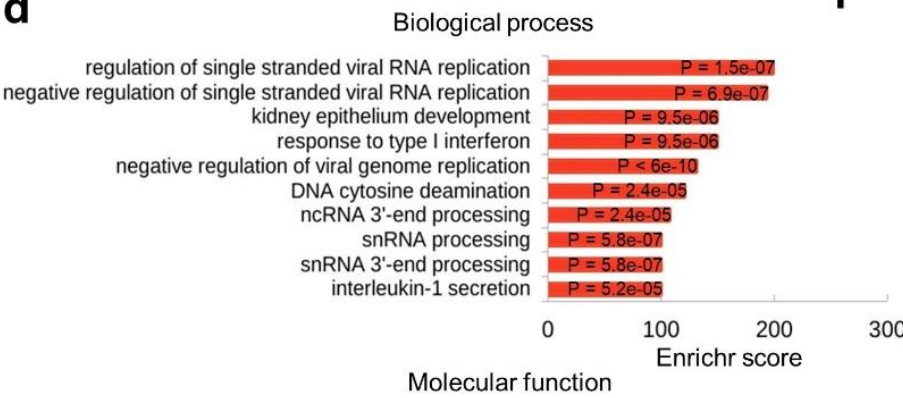

Molecular function

natural killer cell lectin-like receptor binding bile acid binding
ketosteroid monoxygenase activity protein binding involved in cell-matrix adhesion $P=2.7 \mathrm{e}-04$ collagen binding oxidoreductase activity oxidoreductase activity, oxidizing metal ions and NAD transforming growth factor beta binding $\mathrm{N}$-acetylglucosamine 6-O-sulfotransferase activity
type II transforming growth factor beta receptor binding b

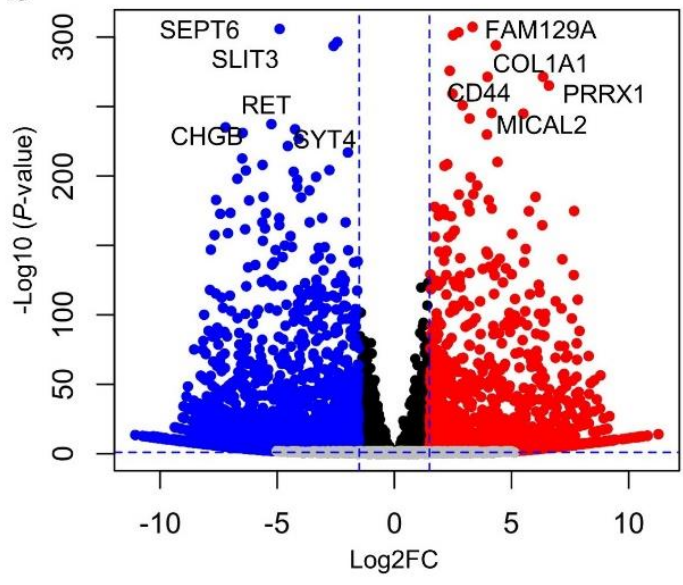

e

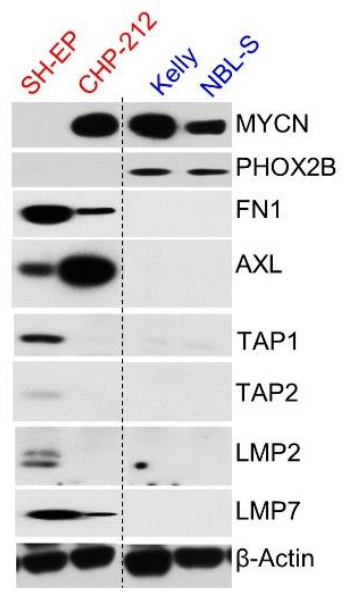

f

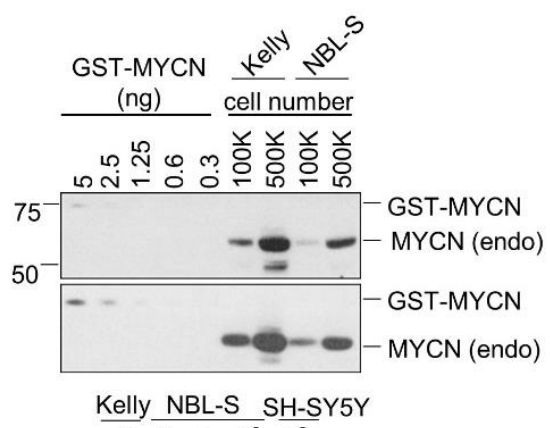

WCE $( \mu \mathrm { g } ) 2 \longdiv { 2 5 } 2 5 5 0 \quad 1 0 0,00$

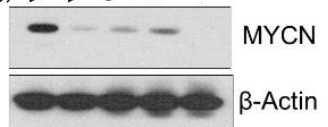


Sengupta et al.

Supplementary Fig. 5. Mesenchymal lineage-specific marker and cell-intrinsic immune gene expression are significantly correlated in NB. (a) Scatter plot of adrenergic and mesenchymal scores in NB cell lines (GSE28019). (b) Volcano plot showing the gene expression

408 changes between mesenchymal SH-EP and adrenergic SH-SY5Y cells. The top ten lineage

409 marker genes are highlighted. The fold changes are represented in $\log _{2}$ scale (X-axis) and the -

$410 \log _{10}$ of the $P$-values depicted on $Y$-axis $\left(F D R<0.1\right.$ and $\log _{2} F C>1$ ). (c) Venn diagram of the

411 overlap between DEGs in SH-EP cells (compared to SH-SY5Y cells) and the mesenchymal or

412 adrenergic signatures derived from Groningen et $\mathrm{al}^{38}$. Statistical significance was determined

413 using Fisher's exact test. (d) GO analysis of differentially upregulated genes in mesenchymal SH-

414 EP compared to adrenergic SH-SY5Y cells. (e) WB analysis of cell lineage marker and antigen

415 processing gene expression in the indicated NB cells. Dotted line indicates the margin where gel

416 images have been cut. (f) Upper, WB analysis of MYCN levels in the indicated numbers of Kelly

417 and NBL-S NB cells titrated against known amounts of purified GST-MYCN protein. Lower, WB

418 analysis comparing MYCN levels in whole cell extracts (WCE) from Kelly cells to titrated levels

419 from WCE in NBL-S cells. SH-SY5Y cells that do not express MYCN serve as a negative control.

420 Actin was used as a loading control in (e) and (f). 
Sengupta et al.

421 with our observations in primary tumors, cell lines with higher mesenchymal gene signatures

422 grouped together and had significantly higher expression of tumor cell-intrinsic immune genes,

423 compared with the remainder, which had higher adrenergic scores and were mostly associated

424 with reduced immune marker gene expression (Fig. 3a, b). To further understand the association

425 of tumor cell-intrinsic immune pathways with lineage state, we focused on two neuroblastoma cell

426 lines -SH-SY5Y and SH-EP - subclones of the MYCN-nonamplified SK-N-SH cell line separated

427 on the basis of neuroblastic versus substrate-adherent morphology ${ }^{45}$ and determined to be

428 adrenergic and mesenchymal ${ }^{38}$, respectively. RNA sequencing showed that the differentially up-

429 and down-regulated genes in SH-EP compared with SH-SY5Y cells significantly overlapped with

430 established signatures of mesenchymal and adrenergic states, thus confirming their respective

431 phenotypes (Supplementary Fig. 5b, c). We noted that genes with roles in eliciting an immune

432 response were among the top differentially upregulated genes in mesenchymal SH-EP cells,

433 especially those involved in antigen processing and presentation and positive regulation of $\mathrm{MHC}$

434 expression (Fig. 3c, d). Moreover, gene ontology (GO) analysis of the upregulated transcripts

435 revealed enrichment for innate and adaptive immune responses including type-I interferon

436 signaling and ligands for the NK cell receptor, NKG2D (NK cell lectin-like receptor, KLRK1)

437 (Supplementary Fig. 5d). By contrast, adrenergic SH-SY5Y cells that showed upregulation of

438 neuronal lineage markers did not show significant enrichment of immune function genes (Fig.

439 3d), providing further evidence that cell-intrinsic immunogenicity is associated with the

440 mesenchymal phenotype.

441 The absence of a productive immune response has often been described in MYCN-

442 amplified neuroblastoma tumors ${ }^{24,25}$; indeed, the vast majority of such tumors in our cohort

443 exhibited similar findings (Supplementary Fig. 2a, b). Nonetheless, based on our intriguing

444 finding of upregulation of immune response genes in a small number of $M Y C N$-amplified tumors

445 (Supplementary Fig. 2a, b) that possessed mesenchymal cell signatures (Fig. 2e), we sought

446 to understand the role of MYCN in mediating this immune response. We used Kelly and CHP-212 
Sengupta et al.

447 human neuroblastoma cells that expressed amplified MYCN but were of adrenergic and

448 mesenchymal phenotypes, respectively ${ }^{37,38}$, and NBL-S cells that lacked MYCN amplification but

449 expressed moderate levels of MYCN RNA and protein $^{46}$ (Supplementary Fig. 5e) and were

450 classified as adrenergic (van Groningen et al., 2017 and this study). RNA-sequencing and flow

451 cytometry analysis suggested that tumor cell-intrinsic immune genes involved in antigen

452 processing and $\mathrm{MHC}$ regulation were highly expressed in mesenchymal CHP-212 compared to

453 adrenergic Kelly and NBL-S cells (Fig. 3e, f; supplementary fig. 5e). Importantly, although

454 MYCN expression in NBL-S cells was lower than in Kelly cells (Supplementary Fig. 5f), these

455 immune transcripts were expressed at lower levels in both cell lines, consistent with their

456 adrenergic status (Fig. 3e; Supplementary Fig. 5f). To further verify that cell state dictate tumor

457 cell-intrinsic immunogenicity, we depleted MYCN expression in Kelly cells and observed no

458 significant change to the IFN- $\gamma$-induced expression of HLA and antigen processing genes

459 compared to control cells (Fig. 3g). Thus, our findings suggest that the lineage state of

460 neuroblastoma cells specifies the expression of tumor cell-intrinsic immune marker genes.

461

462 Cellular reprogramming to the mesenchymal state leads to increased immunogenicity

463 We next questioned whether acquisition of the mesenchymal phenotype would be sufficient to

464 render adrenergic neuroblastoma cells immunogenic. One of the top overexpressed genes in $\mathrm{SH}$ -

465 EP mesenchymal cells, $P R R X 1$, encodes a core lineage-specific homeobox transcription factor

466 (TF), whose overexpression induces the mesenchymal state in neuroblastoma cells ${ }^{37,38}$

467 (Supplementary Fig. 5b). We therefore overexpressed doxycycline-inducible $P R R X 1$ in

468 adrenergic SH-SY5Y cells and observed a gradual loss of the adrenergic lineage marker

469 PHOX2B, together with increased expression of the mesenchymal markers, fibronectin, vimentin

470 and AXL (Fig. 4a; Supplementary Fig. 6a). By contrast, overexpression of DNA-binding mutants 
Sengupta et al.

\section{Fig. 4.}

\section{a}

Days $\frac{5}{-+} \frac{9}{-+} \frac{14}{-+} \frac{19}{-+} \frac{24}{-+} \frac{29}{-+}$

C
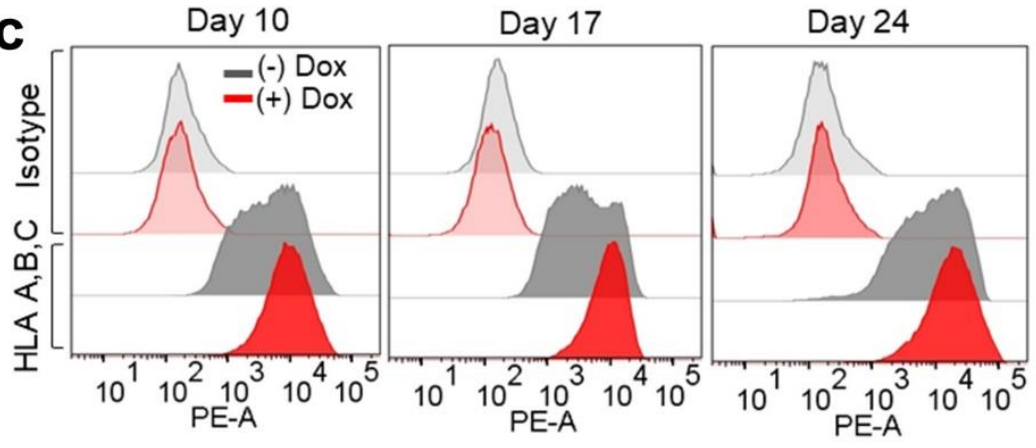

DB mutants

WT $\Delta \mathrm{H} 1$

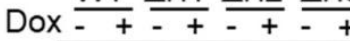

b
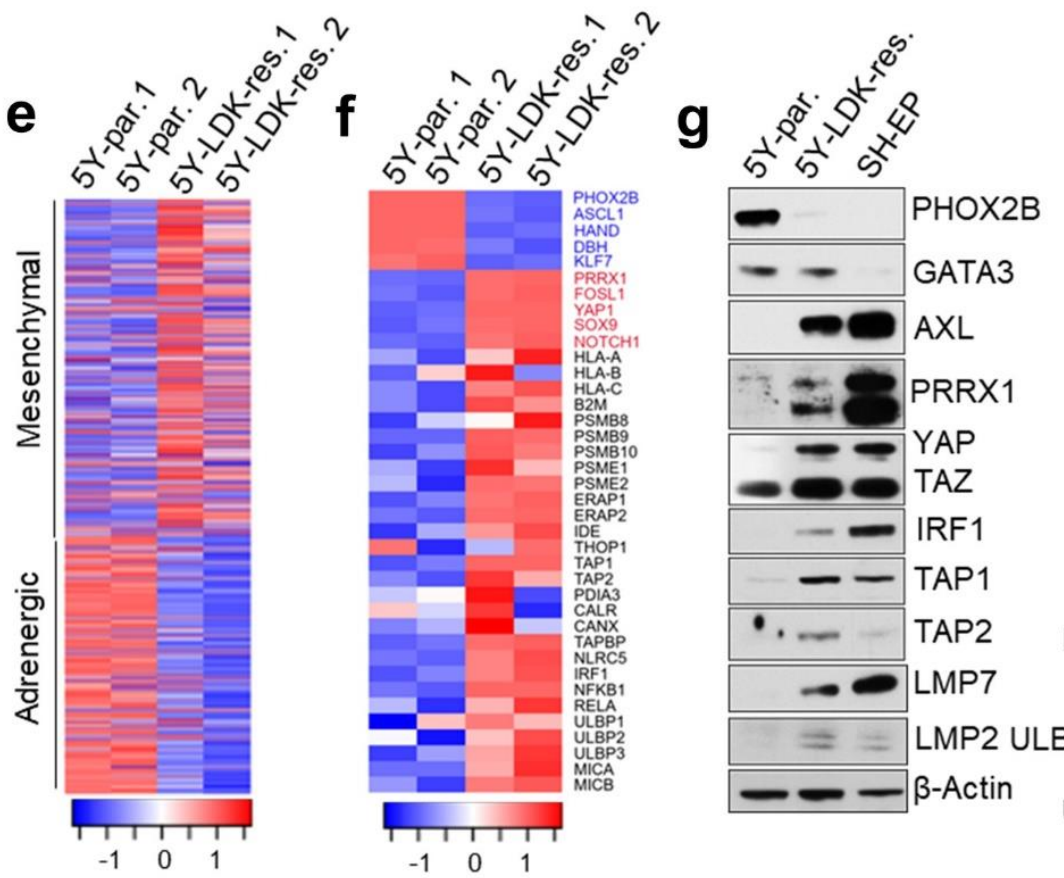
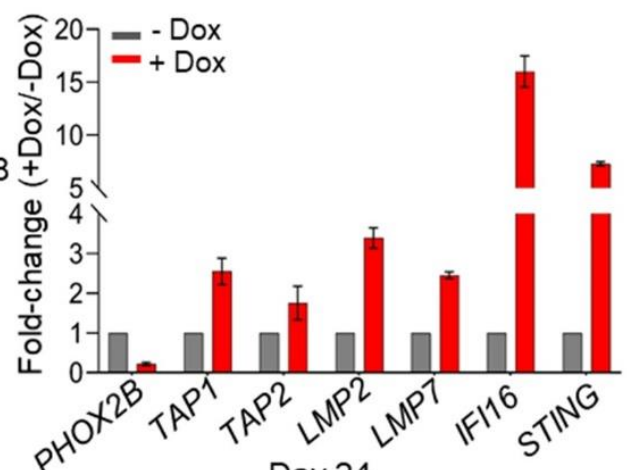

d

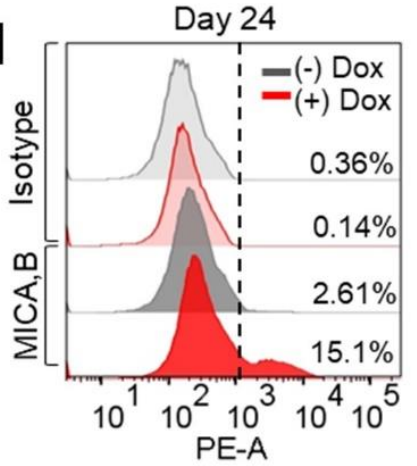

h

$-5 Y$-par. - 5Y-LDK-res. - SH-EP
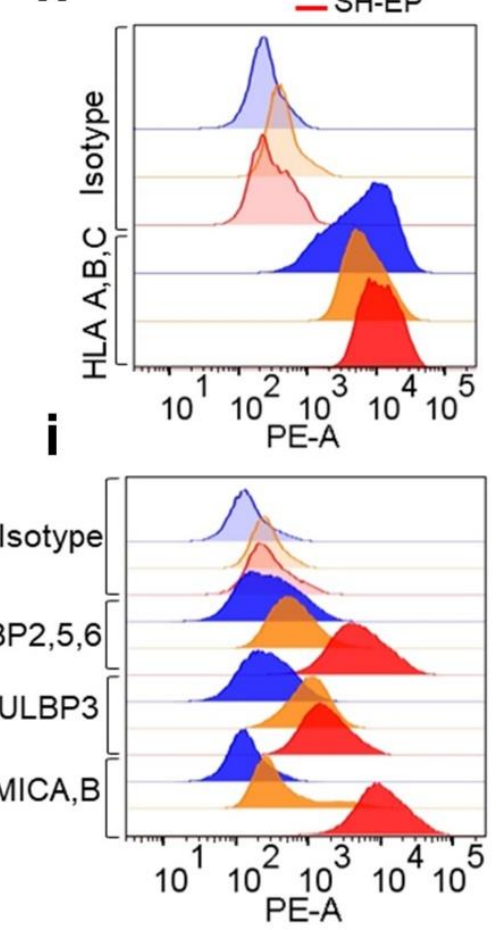
Sengupta et al.

471 Fig. 4. Reprogramming of adrenergic NB cells to the mesenchymal cell state leads to

472 increased expression of immune response genes. (a) Left, WB analysis of PHOX2B and

473 antigen processing gene expression in adrenergic SH-SY5Y cells engineered to express

474 doxycycline (dox)-inducible PRRX1 in the presence or absence of dox $(200 \mathrm{ng} / \mathrm{mL})$ at the

475 indicated time points. Right, WB analysis of the indicated proteins in SH-SY5Y cells expressing

476 dox-inducible wild-type (WT) or DNA-binding mutants of PRRX1 at 10 days post dox-induction.

477 The DNA-binding (DB) mutants harbor individual deletions of the three $\alpha$-helices $(\Delta \mathrm{H} 1, \Delta \mathrm{H} 2$ and

$478 \Delta \mathrm{H} 3$ ) within the PRRX1 homeodomain. (b) RT-qPCR analysis of the indicated immune response

479 genes in the same cells as in (a). Data represent the means $\pm S D, n=2$ biological replicates. (c)

480 FACS analysis of cell surface HLA expression following dox-inducible expression of PRRX1 in

481 SH-SY5Y cells at the indicated time points. Data are representative of 2-3 independent

482 experiments. (d) FACS analysis of cell surface MICA/MICB expression after PRRX1 induction for

48324 days in the same cells as in (c). A logscale expression value of $10^{3}$ was used as a threshold

484 (vertical line) to gate MICA/MICB negative $\left(<10^{3}\right)$ and positive $\left(\geq 10^{3}\right)$ populations. Numbers on

485 the right indicate the percentage of MICA/MICB-positive cells. Plots are representative of 2

486 independent experiments. (e) Heat map representation of adrenergic and mesenchymal gene

487 signatures in parental (5Y-par) and LDK-resistant (5Y-LDK-res) SH-SY5Y cells ( $n=2$ biological

488 replicates). Rows represent z-scores of $\log _{2}$ expression values for each gene in both cell types.

489 (f) Heat map depicting the expression of cell lineage markers (blue, adrenergic; red,

490 mesenchymal), antigen processing machinery genes and NKG2D ligands (black) in parental and

491 LDK-resistant SH-SY5Y cells ( $n=2$ biological replicates). Rows represent z-scores of $\log _{2}$

492 expression values. (g) WB analysis of lineage marker and antigen processing gene expression in

493 the indicated cells. Actin was used as a loading control in all immunoblots. (h, i) FACS analysis

494 of cell surface HLA (h) and NKG2D ligand (i) expression in the indicated cells. 
bioRxiv preprint doi: https://doi.org/10.1101/2021.01.29.428154; this version posted January 30, 2021. The copyright holder for this preprint (which was not certified by peer review) is the author/funder. All rights reserved. No reuse allowed without permission.

Sengupta et al.

\section{Supplementary Fig. 6.}

a

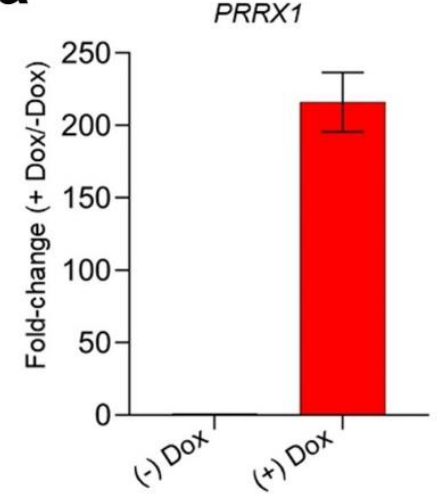

b

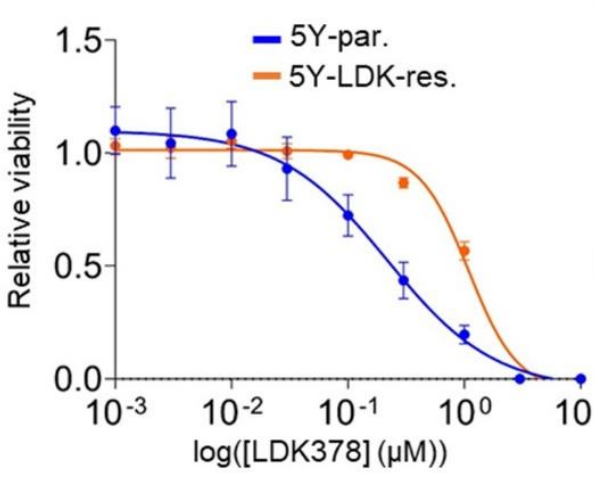

C

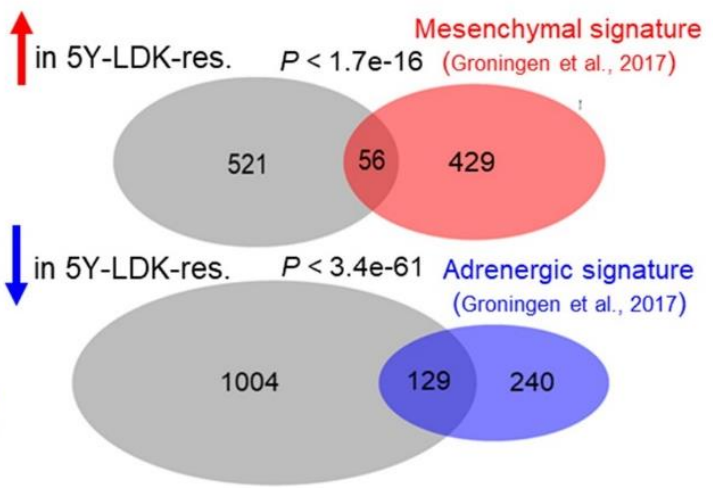

d

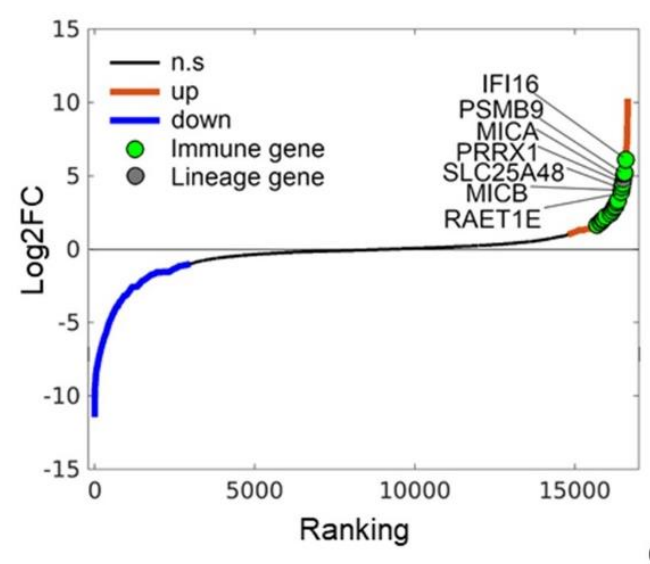

e

\section{Biological process}

negative regulation of protein processing mesodermal cell differentiation antigen processing and presentation embryonic skeletal joint development negative regulation of protein maturation positive regulation of glial cell differentiation regulation of single stranded viral RNA replication negative regulation of chemotaxis

negative regulation of myotube differentiation regulation of toll-like receptor 3 signaling pathway

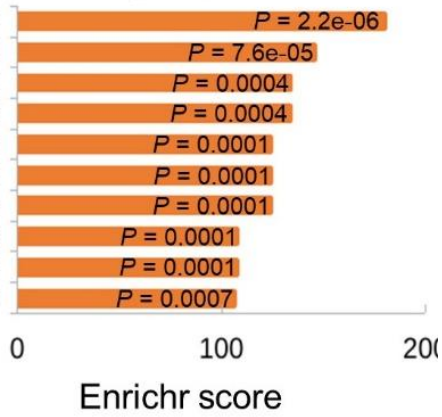

Molecular function

oxidoreductase activity, oxidizing metal ions and NAD collagen binding $\mathrm{N}$-acetylglucosamine 6-O-sulfotransferase activity tumor necrosis factor-activated receptor activity death receptor activity platelet-derived growth factor receptor binding natural killer cell lectin-like receptor binding phosphatidylcholine 1-acylhydrolase activity metalloaminopeptidase activity aminopeptidase activity

\begin{tabular}{|c|}
\hline$P=0.0001$ \\
$P=2.1 \mathrm{e}-07$ \\
$P=0.0007$ \\
$P=4.8 \mathrm{e}-05$ \\
$P=4.8 \mathrm{e}-05$ \\
$P=0.0003$ \\
\hline$P=0.001$ \\
\hline$P=0.002$ \\
\hline$P=0.001$ \\
\hline$P=0.0003$ \\
\hline 50 \\
Enrichr score
\end{tabular}



cells is associated with reprogramming to the mesenchymal lineage and increased expression of immune response genes. (a) RT-qPCR analysis of PRRX1 expression in

499 adrenergic SH-SY5Y cells engineered to express doxycycline (dox)-inducible PRRX1 in the 500 presence or absence of dox $(200 \mathrm{ng} / \mathrm{mL})$ for 10 days. Data represent the means $\pm \mathrm{SD}, n=2$ 501 biological replicates. (b) Dose-response curves of ceritinib (LDK378)-sensitive (5Y-par.) and 502 resistant (5Y-LDK-res.) SH-SY5Y cells treated with increasing concentrations of LDK378 for 72 503 h. Data represent means \pm SD, $n=2$ biological replicates. (c) Venn diagrams depicting the overlap 504 between the DEGs in LDK-resistant SH-SY5Y cells (compared to parental SH-SY5Y cells) and 505 the mesenchymal or adrenergic signatures derived from Groningen et $\mathrm{al}^{38}$. $P$-values were 506 determined by Fisher's exact test. (d) Waterfall plot of the fold-change in RNA expression levels 507 of up- and down-regulated genes in LDK-resistant SH-SY5Y cells compared to parental SH-SY5Y 508 cells; selected immune genes are highlighted in green. (e) GO analysis of differentially 509 upregulated genes in LDK-resistant SH-SY5Y compared to parental SH-SY5Y cells. 
Sengupta et al.

510 of $P R R X 1$ that contained homeodomain deletions had no effect on mesenchymal marker

511 expression, indicating that the lineage switch was a direct consequence of PRRX1-mediated

512 transcriptional control (Fig. 4a). Next, to determine whether the phenotypic switch had any effect

513 on tumor cell-intrinsic pro-inflammatory pathways, we analyzed the expression of genes involved

514 in antigen processing (TAP1, TAP2, LMP2, LMP7) as well as IFI16 and STING (TMEM173),

515 innate immune regulators that were differentially upregulated in mesenchymal NB cells (Fig. 3c).

516 Induction of wild-type (WT) PRRX1 led to increased RNA expression of these genes (Fig. 4b).

517 Moreover, WT PRRX1 but not its DNA-binding mutants led to increased TAP1 and LMP7 protein

518 expression, which was accompanied by a sustained increase in cell surface MHC expression

519 (Fig. 4a, c). Additionally, PRRX1 induction led to increased cell surface expression of MICA and

520 MICB, ligands for the activating NK cell receptor NKG2D, in a minor population of cells (Fig. 4d),

521 in agreement with elevated expression of these proteins in mesenchymal neuroblastoma cells

522 (Fig. 3c). These results suggest that conversion from the adrenergic to mesenchymal cell state

523 may be adequate to reprogram immune-insensitive cells toward immunocompetency.

Transition from the adrenergic to the mesenchymal state in neuroblastoma is

525 accompanied by resistance to chemotherapy ${ }^{38}$. Whether this transition in the face of treatment

526 pressure might include the acquisition of a pro-inflammatory signature is unclear, leading us to

527 compare adrenergic neuroblastoma cells that had gained mesenchymal features during the

528 development of treatment resistance with their sensitive, adrenergic counterparts. For this

529 purpose, we used an isogenic pair of cell lines comprising adrenergic SH-SY5Y neuroblastoma

530 cells that express the $A L K^{F 1174 L}$ mutation and are sensitive to the small molecule inhibitor ceritinib

531 (LDK378) (parental SH-SY5Y, IC $50=150 \mathrm{nM}$ ), and their ceritinib-resistant derivatives (LDK-

532 resistant SH-SY5Y, IC $50=1101 \mathrm{nM}$ ) (Supplementary Fig. 6b) ${ }^{47}$. Comparison of the gene

533 expression signatures of these cell lines revealed significant downregulation of adrenergic

534 transcripts in LDK-resistant SH-SY5Y cells with concomitant upregulation of the mesenchymal 
Sengupta et al.

535 signature (Fig. 4e). Moreover, a significant overlap was noted between the differentially up- or

536 downregulated transcripts in the LDK-resistant SH-SY5Y cells and established signatures of

537 mesenchymal and adrenergic states, respectively (Supplementary Fig. 6c), suggesting that

538 these cells had acquired features of the mesenchymal phenotype with resistance. Consistent with

539 the key role of PRRX1 in triggering the conversion from an adrenergic to mesenchymal cell state,

540 we observed that this TF was among the top upregulated genes in LDK-resistant SH-SY5Y cells

541 (Supplementary Fig. 6d). The mesenchymal state of the LDK-resistant SH-SY5Y cells was

542 further supported by the loss of the pivotal adrenergic marker, PHOX2B, and increased

543 expression of additional mesenchymal markers AXL, YAP, TAZ, and IRF1, although these

544 changes were not as pronounced as those in SH-EP mesenchymal cells that served as a positive

545 control (Fig. 4f, g). Further evidence supporting the conversion to the mesenchymal state came

546 from the differential upregulation in LDK-resistant SH-SY5Y cells of cell-intrinsic immune markers

547 engaged in antigen processing and presentation and NK cell activating receptor ligands (PSMB9,

548 MICA, MICB); in fact, these were among the top differentially upregulated genes in LDK-resistant

549 SH-SY5Y cells (Supplementary Fig. 6d, e; Fig. 4f, g). These changes in immune genes

550 coincided with increases in cell surface expression of MHC receptors to levels comparable to

551 those in mesenchymal SH-EP cells (Fig. 4h), as well as the increased expression of ligands for

552 the NK cell-activating receptor NKG2D (Fig. 4i). Thus, the genetic reprogramming from the

553 adrenergic to the mesenchymal state that occurred with therapy resistance also led to the

554 upregulation of tumor cell-intrinsic pro-inflammatory pathway genes suggesting that such

555 conversion could render the tumor cells susceptible to recognition by $\mathrm{T}$ and NK cells. 
Sengupta et al.

557 Immune response gene expression during cell state transition is epigenetically regulated

558 As lineage plasticity in neuroblastoma is epigenetically driven $37,38,48,49$, we next questioned 559 whether the altered expression of immune response genes observed in the individual cell states 560 could be the result of changes in chromatin organization. To this end, we analyzed the chromatin

561 occupancies of active and repressive histone marks at immune genes that were upregulated in

562 adrenergic SH-SY5Y cells upon induction of PRRX1 (Fig. 4b). Indeed, PRRX1 induction resulted

563 in increased binding of the active H3K4me3 mark as well as loss of repressive H3K27me3 binding

564 at several candidate immune genes, including the APM genes TAP1 and PSMB9 (Fig. 5a;

565 Supplementary Fig. 7a). To understand epigenetic modifications that occur during the 566 spontaneous transition between the two lineage states (as compared with forced expression of

$567 P R R X I$ ) on a genome-wide basis, we compared histone occupancies between adrenergic 568 (parental SH-SY5Y) cells and those that had acquired mesenchymal characteristics with drug 569 resistance (LDK-resistant SH-SY5Y) (Fig. 4e), using SH-EP cells as a typical example of the 570 mesenchymal state. ChIP-seq analysis of active H3K27ac binding identified that the super571 enhancers (SEs) in LDK-resistant SH-SY5Y cells were associated with genes that conferred 572 mesenchymal identity while parental SH-SY5Y cells retained SEs at genes that conferred 573 adrenergic identity (Supplementary Fig. 7b), consistent with evidence that lineage plasticity is 574 driven by cell type-specific SEs ${ }^{37,38}$. To determine whether the SE-mediated regulation of lineage 575 genes also extended to genes associated with immune responsiveness, we analyzed the genes 576 in our 41-gene immune activation signature (Supplementary Table 3;Fig. 1e) as well as those 577 associated with an IFN-response signature $(n=91)$ in primary tumors and cell lines (Fig. 1b;

578 Supplementary Fig. 5d, see Methods). Despite the higher expression of these genes in 579 mesenchymal cells (LDK-resistant SH-SY5Y and SH-EP), none was associated with an SE, 580 prompting us to focus on the promoter regions. We observed significantly higher enrichment of $581 \mathrm{H} 3 \mathrm{~K} 27 \mathrm{ac}$ and $\mathrm{H} 3 \mathrm{~K} 4 \mathrm{me} 3$ binding at regions spanning the transcription start sites (TSS $\pm 2 \mathrm{~kb}$ ) at 582 cell-intrinsic immune genes in LDK-resistant SH-SY5Y and SHEP compared to parental SH-SY5Y 
bioRxiv preprint doi: https://doi.org/10.1101/2021.01.29.428154; this version posted January 30, 2021. The copyright holder for this preprint (which was not certified by peer review) is the author/funder. All rights reserved. No reuse allowed without permission.

Sengupta et al.

Fig. 5
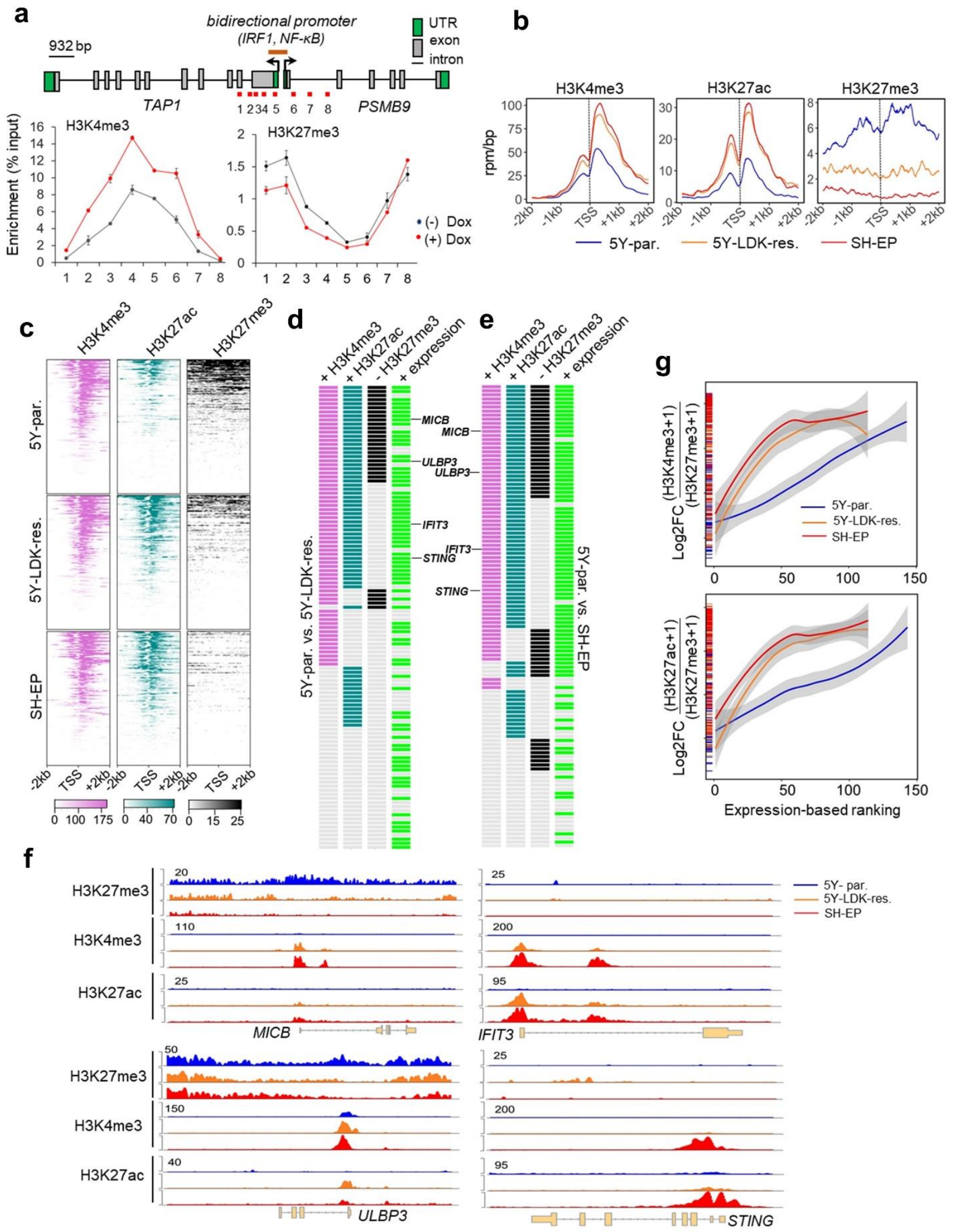
Sengupta et al.

Fig. 5. Activation of immune gene expression associated with cell state transition is epigenetically regulated. (a) Upper, Linear representation of TAP1 and PSMB9 gene loci showing the locations of the bidirectional promoter and IRF1 and NF-KB binding sites. The amplicons (1-8) analyzed for histone mark occupancy are shown in red. Lower, ChIP-qPCR

587 analysis of $\mathrm{H} 3 \mathrm{~K} 4 \mathrm{me} 3$ and $\mathrm{H} 3 \mathrm{~K} 27$ me3 enrichment at the indicated amplicons along the 588 TAP1/PSMB9 locus in adrenergic SH-SY5Y cells expressing dox-inducible PRRX1 in the 589 presence or absence of dox $(200 \mathrm{ng} / \mathrm{mL})$ for 10 days. Data represent the means $\pm \mathrm{SD}, n=2$ 590 biological replicates. (b) Metagene representations of average ChIP-seq occupancies of the 591 indicated histone marks at the promoters of tumor cell-intrinsic immune response genes (TSS \pm $5922 \mathrm{~kb} ; \mathrm{n}=134)$ in parental (5Y-par.), LDK-resistant (5Y-LDK-res.) SH-SY5Y and SH-EP NB cells.

593 (c) Heat map representation of histone enrichment at the same immune gene promoters as in 594 (b), ranked in decreasing order of occupancy in the indicated cells. Each row represents the 595 normalized densities of histone marks within a $\pm 2 \mathrm{~kb}$ window centered on the TSS. (d, e) 596 Representation of pairwise comparisons between parental SH-SY5Y and LDK-resistant SH-SY5Y 597 (d), and parental SH-SY5Y and SH-EP cells (e). The changes (+, gained; -, lost) in occupancies 598 of the active (H3K4me3, H3K27ac) and repressive (H3K27me3) histone marks $\left(\log _{2} \mathrm{FC} \geq 0.75\right.$, 599 TSS $\pm 2 \mathrm{~kb}$ ), together with the corresponding changes in RNA expression (+, overexpressed; $\log _{2}$ 600 FC $\geq 1$ ) of each of the 134 tumor cell-intrinsic immune genes analyzed in (b) are shown. 601 Representative genes showing either a switch from repressive to active chromatin (MICB, ULBP3) 602 or associated only with a gain of active chromatin (IFIT3, STING) are shown. (f) ChIP-seq tracks 603 depicting the gain of active histone binding together with the loss of repressive histone binding 604 (left) or gain of active marks without changes in repressive mark occupancy (right) at the indicated 605 immune gene loci. Signal intensity is given at the top left corner for each track. (g) Loess 606 regression analysis of the correlation between the ratios of active to repressive histone binding at 607 the promoters (TSS $\pm 2 \mathrm{~kb}$ ) of immune response genes and their RNA expression (Upper, 
bioRxiv preprint doi: https://doi.org/10.1101/2021.01.29.428154; this version posted January 30, 2021. The copyright holder for this preprint (which was not certified by peer review) is the author/funder. All rights reserved. No reuse allowed without permission.

Sengupta et al.

608 H3K4me3:H3K27me3; Lower, H3K27ac:H3K27me3). Genes are ranked based on increasing

609 expression. Shaded regions represent 95\% confidence intervals. 
bioRxiv preprint doi: https://doi.org/10.1101/2021.01.29.428154; this version posted January 30, 2021. The copyright holder for this preprint (which was not certified by peer review) is the author/funder. All rights reserved. No reuse allowed without permission.

Sengupta et al.

\section{Supplementary Fig. 7.}

b

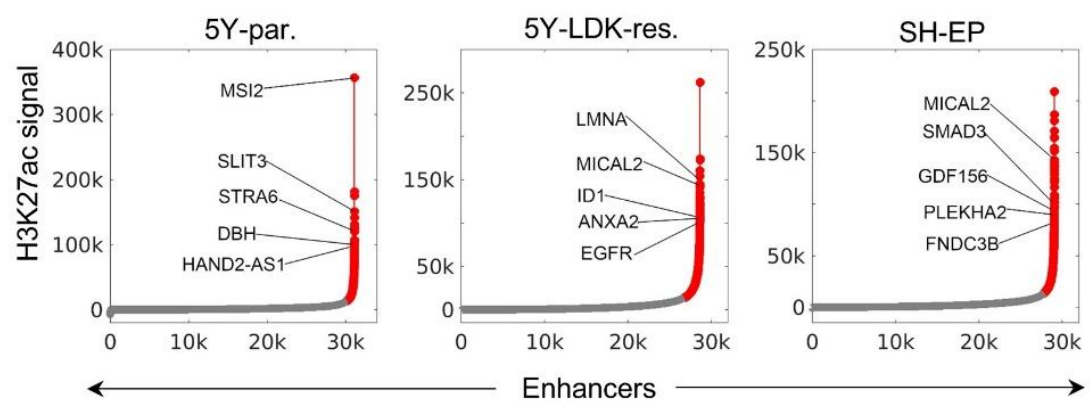

a

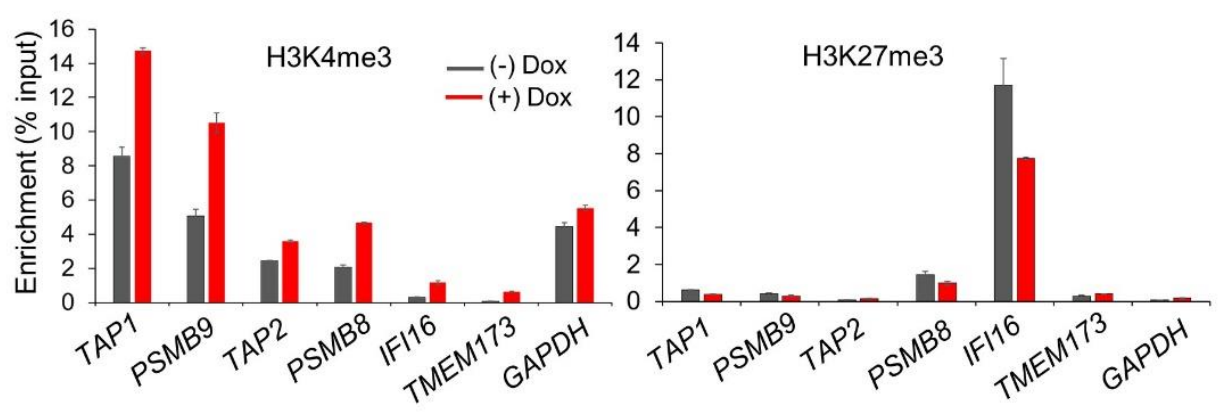

C

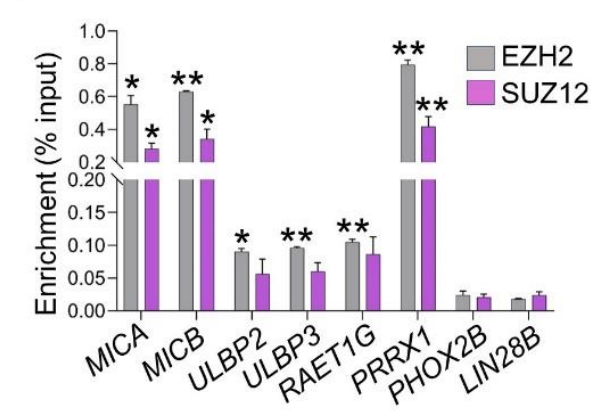

d

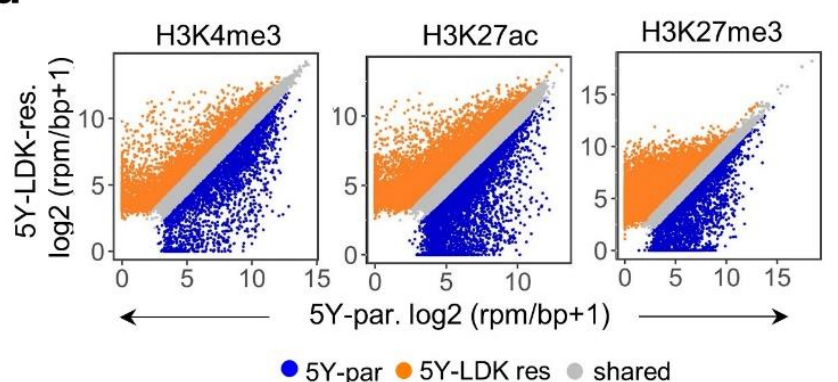

e

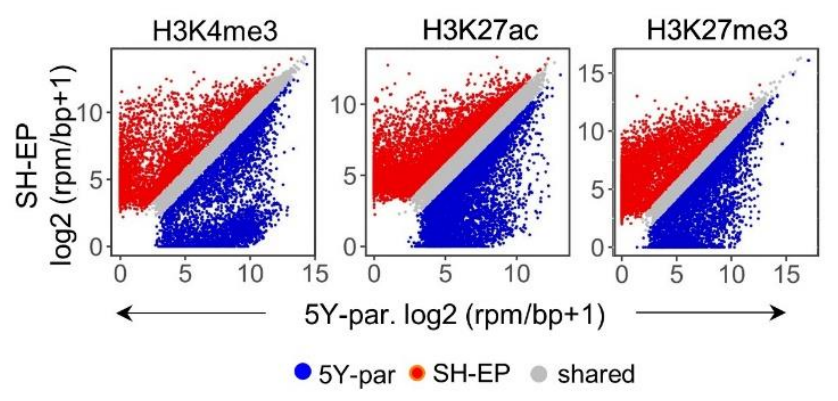

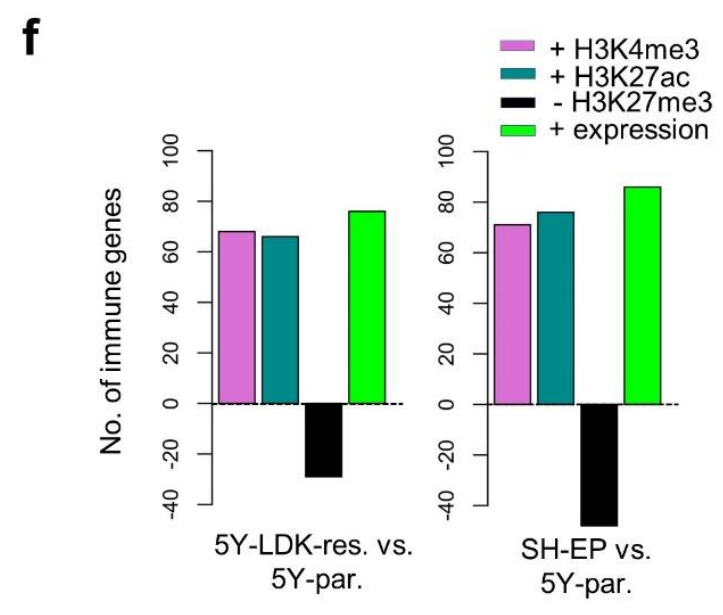

g
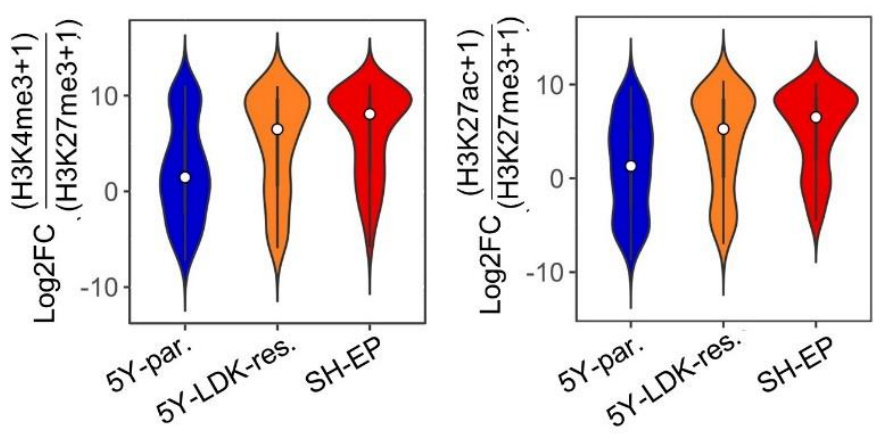
Sengupta et al.

611 Supplementary Fig. 7. Immune gene activation associated with the mesenchymal cell state

612 is epigenetically regulated. (a) ChIP-qPCR analysis of H3K4me3 and H3K27me3 enrichment

613 at the promoters of the indicated immune genes in SH-SY5Y cells expressing doxycycline-

614 inducible PRRX1 in the presence or absence of dox (200 ng/mL) for 10 days. Enrichments at

615 TAP1 and PSMB9 loci correspond to amplicons 4 and 6 respectively, as described in Fig. 5a.

616 Data represent the means $\pm \mathrm{SD}, n=2$ biological replicates. (b) Identification of enhancer regions

617 in parental SH-SY5Y, LDK-resistant SH-SY5Y and SH-EP cells. H3K27ac bound regions

618 identified as significant peaks were stitched together if they were within $12.5 \mathrm{~kb}$ of each other and

619 termed typical enhancers (plotted in grey). Super enhancers (SEs) were defined as stitched

620 enhancers surpassing the threshold signal based on the inclination point in all cell types (plotted

621 in red). In parental SH-SY5Y, LDK-resistant and SH-EP cells, $2.94 \%(915 / 31116), 6.56 \%$

$622(1880 / 28635)$ and 4.18\% (1215/29057) of the enhancers were classified as SEs respectively. The

623 top five SE-associated lineage-specific genes are highlighted. (c) ChIP-qPCR analysis of EZH2

624 and SUZ12 enrichment at the indicated genes in adrenergic 5Y-par. cells. Data represent the 625 means $\pm \mathrm{SD}, n=2$ biological replicates, ${ }^{*} P<0.05 ;{ }^{* *} P<0.01$ two-tailed Student's t-test. $P$-values

626 were calculated in comparison to enrichment observed at the Lin28B TSS (negative control

627 locus). (d, e) Scatter plots representing the differential binding of the indicated histone marks at

628 the promoter regions (TSS $\pm 2 \mathrm{~kb}$ ) of all protein coding genes between parental SH-SY5Y (5Y-

629 par.) and LDK-resistant SH-SY5Y (5Y-LDK res.) (d), and parental SH-SY5Y and SH-EP cells (e).

$630 \mathrm{rpm} / \mathrm{bp}$, reads per million mapped reads per base pair. $A \geq 0.75 \log _{2} \mathrm{FC}$ threshold was used to

631 identify unique peaks for each individual histone mark. Unique and shared peaks are shown in

632 different colors. (f) Bar plots representing the numbers of immune genes with increased

633 deposition of H3K4me3 and H3K27ac $\left(\log _{2} \mathrm{FC} \geq 0.75\right.$, TSS $\left.\pm 2 \mathrm{~kb}\right)$ and loss of H3K27me3 $\left(\log _{2}\right.$

$634 \mathrm{FC} \geq 0.75, \mathrm{TSS} \pm 2 \mathrm{~kb})$ histone marks, together with increased RNA expression $\left(\log _{2} \mathrm{FC} \geq 1\right)$ in

635 mesenchymal LDK-resistant SH-SY5Y (left) or SH-EP (right) as compared to adrenergic parental

636 SH-SY5Y cells. (g) Violin plots of the ratios of active to repressive histone marks (left, 
bioRxiv preprint doi: https://doi.org/10.1101/2021.01.29.428154; this version posted January 30, 2021. The copyright holder for this preprint (which was not certified by peer review) is the author/funder. All rights reserved. No reuse allowed without permission.

Sengupta et al.

637 H3K4me3:H3K27me3; right, H3K27ac:H3K27me3) surrounding immune gene promoters (TSS \pm

$6382 \mathrm{~kb}$ ) in parental SH-SY5Y, LDK-resistant SH-SY5Y and SH-EP cells. Significance was 639 determined by the two-sided Wilcoxon rank-sum test. 
Sengupta et al.

640

641

642

643

644

645

646

647

648

649

650

651

652

653

654

655

656

657

658

659

660

661

662

663

664

665

cells (Fig. 5b, c). On the other hand, the adrenergic parental SH-SY5Y cells showed significantly higher occupancies of the H3K27me3 repressor mark at these immune gene promoters (Fig. $\mathbf{5 b}$, c). Analysis of the polycomb repressive complex 2 (PRC2) that promotes H3K27me3 deposition at repressed chromatin ${ }^{50}$ revealed that immune response genes enriched for $\mathrm{H} 3 \mathrm{~K} 27 \mathrm{me} 3$ binding, such as those encoding the NKG2D ligands MICA/B, ULBP2/3 and RAET1G had significantly higher occupancies for PRC2 subunits, EZH2 and SUZ12 in adrenergic parental SH-SY5Y cells compared to negative control regions that lacked H3K27me3 binding (PHOX2B and LIN28B) (Supplementary Fig. 7c), suggesting active immune gene repression in these cells.

.

We next sought to understand whether the activation of immune response genes observed during the cell state transition from sensitivity to resistance represented a switch from repressive to active chromatin or a gain of active chromatin marks. To this end, we quantified the changes in histone binding occupancies between adrenergic parental SH-SY5Y and mesenchymal LDKresistant SH-SY5Y or SH-EP cells using pair-wise comparisons (Supplementary Fig. 7d, e). Compared to parental SH-SY5Y, LDK-resistant SH-SY5Y and SH-EP cells gained significant H3K4me3 binding at the promoters of $60 \%$ and $62 \%$ immune genes (68 and 71 of 114) respectively, which corresponded with their increased expression (Fig. 5d, e; Supplementary Fig. 7f). A similar significant enrichment of the H3K27ac histone mark was observed at the promoters of these immune genes [LDK-resistant SH-SY5Y, 58\% (66/114); SH-EP, 67\% (76/114)]. Interestingly, gain of these active marks was accompanied by a concomitant loss of H3K27me3 repressive histone binding at the promoters of $25 \%$ and $35 \%$ of (29 and 40 of 114 ) immune genes in LDK-resistant SH-SY5Y and SH-EP cells respectively, as represented by the NKG2D ligands, MICB and ULBP3 (Fig. 5d-f; Supplementary Fig. 7f). On the other hand, in LDK-resistant SH-SY5Y and SH-EP mesenchymal cells, $48 \%$ and $41 \%$ (55 and 47 of 114) immune genes, such as the IFN-regulated factors IFIT3 and STING, gained either one or both active marks without changes in occupancy of the repressive mark (Fig. 5d-f; Supplementary 
Sengupta et al.

666 Fig. 7f). Furthermore, the mesenchymal cells showed a significantly higher ratio of active to

667 repressor histone binding at the TSSs of immune-related genes (H3K4me3 or

668 H3K27ac/H3K27me3) (Supplementary Fig. 7g), which importantly, also correlated with the

669 increased expression of these genes in this cell state (Fig. $\mathbf{5 g}$ ). Therefore, our results suggest

670 that the immune gene activation observed with the transition from the adrenergic to the

671 mesenchymal cell state represents either a switch from repressive to active chromatin or a gain

672 of active chromatin at promoter regions.

673

\section{Mesenchymal neuroblastoma cells functionally engage cytotoxic T cells}

675 To assess the functional consequences of the increased immunogenicity associated with a 676 mesenchymal phenotype, we utilized the murine neuroblastoma cell line NB-9464, which was 677 derived from tumors arising in the Th-MYCN genetically engineered mouse model (GEMM). This

678 model was generated in immunocompetent C57BL/6 mice and the tumors recapitulate the genetic 679 and immunological features of human neuroblastoma ${ }^{51,52}$. We observed that NB-9464 cells 680 consisted of distinct populations that could be sorted on the basis of surface MHC class I H-2Kb 681 expression into high $\left(\mathrm{H}-2 \mathrm{~Kb}^{\text {hi }}\right)$ - or low $\left(\mathrm{H}-2 \mathrm{~Kb}^{10}\right)$-expressing populations (Supplementary Fig. 8a;

682 Fig. 6a), both of which expressed transgenic human MYCN (Fig. 6b). Consistent with our 683 hypothesis, $\mathrm{H}-2 \mathrm{~Kb}^{\text {hi }}$ cells were enriched for bona fide mesenchymal markers - Prrx1, Sox9, 684 Notch1, and Snai2 (Slug) (Fig. 6b), and showed enhanced migration and invasion, as might be 685 expected from their neural crest cell-like state (Supplementary Fig. 8b, c). Importantly, the 686 mesenchymal $\mathrm{H}-2 \mathrm{~Kb}^{\text {hi }} \mathrm{NB}-9464$ cells, but not their adrenergic $\mathrm{H}-2 \mathrm{~Kb}^{\text {lo }}$ counterparts showed 687 augmented expression of cell surface class I MHC H-2Kb in response to IFN- $\mathrm{Y}$, suggesting that 688 the mesenchymal cells had the potential for inducing a T cell-driven antitumor immune response 689 (Fig. 6c). 
Sengupta et al.

Hence, to determine whether the increased MHC class I expression of mesenchymal versus

691 adrenergic cells translated into T cell activation, we first asked whether mesenchymal $\mathrm{H}-2 \mathrm{~Kb}^{\text {hi }}$

692 NB-9464 cells were capable of exogenous antigen presentation. Using the well-characterized

693 chicken ovalbumin-derived peptide $\left(\mathrm{OVA}_{257-264}\right.$ or SIINFEKL) antigen that binds to $\mathrm{H}-2 \mathrm{~Kb}$ and can

694 be recognized by specific T cell receptors (TCRs) on $\mathrm{CD}^{+} \mathrm{T}$ cells ${ }^{53}$, we found that in comparison

695 to $\mathrm{H}-2 \mathrm{~Kb}^{10}$ cells, $\mathrm{H}-2 \mathrm{~Kb}^{\text {hi }}$ cells expressed significantly higher levels of the $\mathrm{H}-2 \mathrm{~Kb}-\mathrm{SIINFEKL}$

696 complex (Fig. 6d). Next, we determined whether antigen presentation through H-2Kb enables

697 mesenchymal tumor cells to be recognized by antigen-specific T cell receptors (TCRs) on CD8 ${ }^{+}$

698 T cells, the first step towards a cytotoxic response. For this purpose, we used OT-I CD8 ${ }^{+}$T cells

699 from C57BL/6 mice expressing a transgenic TCR that specifically recognizes the $\mathrm{H}-2 \mathrm{~Kb}-$

700 SIINFEKL complex ${ }^{53}$. $\mathrm{H}-2 \mathrm{~Kb}^{\text {hi }}$ or $\mathrm{H}-2 \mathrm{~Kb}^{10}$ cells loaded with the SIINFEKL peptide were cocultured

701 with naïve OT-I cells, after which OT-I activation was measured through cell surface CD69

702 expression, an early marker of T-cell activation ${ }^{54}$. Mesenchymal $\mathrm{H}-2 \mathrm{~Kb}^{\text {hi }}$ cells led to significantly

703 higher OT-I activation in comparison with adrenergic $\mathrm{H}-2 \mathrm{~Kb}^{10}$ cells, indicating specific recognition

704 of the H-2Kb-SIINFEKL complex by the TCR on OT-I cells (Fig. 6e). By contrast, co-cultures of

705 OT-I cells and either $\mathrm{H}-2 \mathrm{~Kb}^{10}$ or $\mathrm{H}-2 \mathrm{~Kb}^{\text {hi }}$ NB-9464 neuroblastoma cells without the SIINFEKL

706 peptide did not lead to T-cell recognition, confirming the specificity of the TCR-antigen interaction

707 (Fig. 6e). Finally, we investigated whether the differential MHC-I expression between adrenergic

708 and mesenchymal neuroblastoma cells influenced tumor growth in vivo through subcutaneous

709 injection of $\mathrm{H}-2 \mathrm{~Kb}^{\text {hi }}$ or $\mathrm{H}-2 \mathrm{~Kb}^{10}$ cells into syngeneic C57BL/6 (H-2Kb haplotype) mice

710 (Supplementary Fig. 8d). We noted an earlier onset of tumor formation with $\mathrm{H}-2 \mathrm{~Kb}^{10}$ cells

711 compared to $\mathrm{H}-2 \mathrm{~Kb}^{\text {hi }}$ cells (Supplementary Fig. 8d). However, once consistent tumor growth

712 was established, growth or survival rates did not change substantially between the two groups

713 (Supplementary Fig. 8e), despite the persistence of higher $\mathrm{H}-2 \mathrm{~Kb}$ and Prrx1 expression in the

$714 \mathrm{H}-2 \mathrm{~Kb}^{\text {hi }}$ tumors compared with the $\mathrm{H}-2 \mathrm{~Kb}^{\text {lo }}$ tumors (Supplementary Fig. 8f,g). While both types

715 of tumor cells had MYCN expression, histologically, in keeping with their adrenergic phenotype 
bioRxiv preprint doi: https://doi.org/10.1101/2021.01.29.428154; this version posted January 30, 2021. The copyright holder for this preprint (which was not certified by peer review) is the author/funder. All rights reserved. No reuse allowed without permission.

Sengupta et al.

Fig. 6.

a

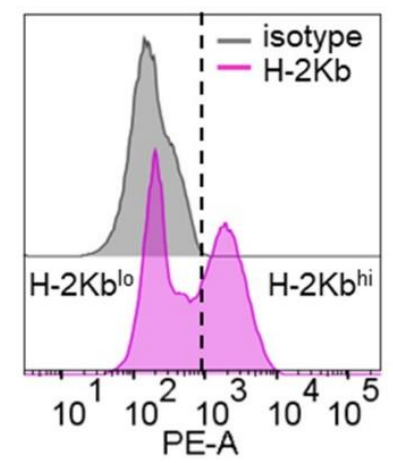

b

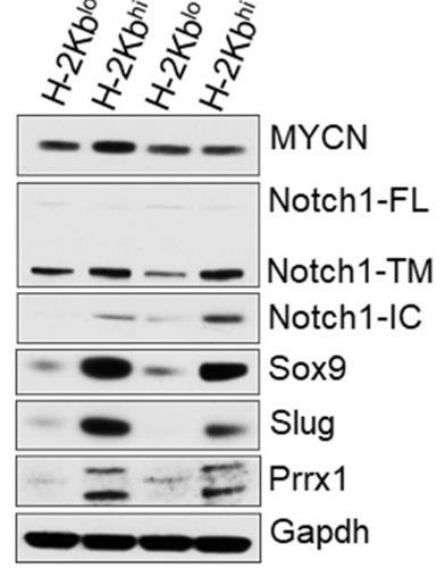

$\bigcup_{\mathrm{H}-2 \mathrm{~Kb}}^{\mathrm{NB}-9464}$

FACS for

H2-Kb-SIINFEKL

complex e

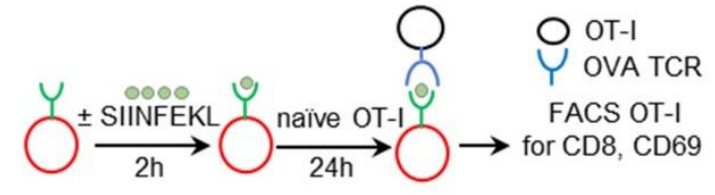

co-culture without OVA

$\bigcirc$ Y OT-I

FACS OT-I

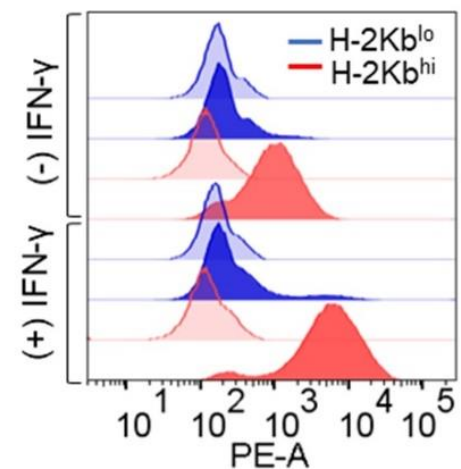

co-culture with OVA
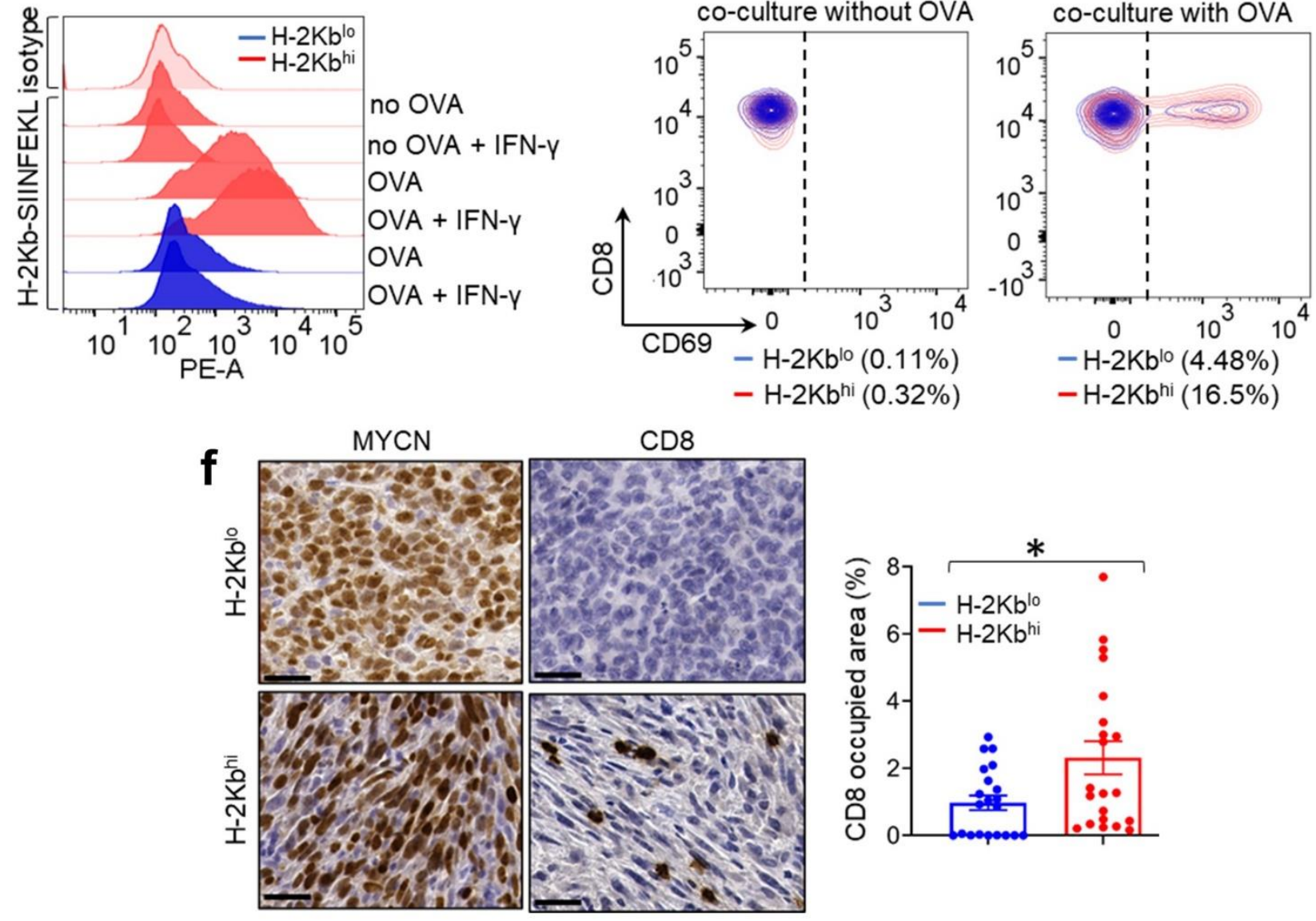
Sengupta et al.

716 Fig. 6. Mesenchymal NB cells functionally engage cytotoxic T cells. (a) FACS analysis of

$717 \mathrm{H}-2 \mathrm{~Kb}$ expression in unsorted NB-9464 cells. Vertical dashed line denotes the logscale

718 expression value used as a threshold to gate $\mathrm{H}-2 \mathrm{~Kb}^{10}$ and $\mathrm{H}-2 \mathrm{~Kb}^{\text {hi }}$ cell populations. (b) WB

719 analysis of the indicated lineage markers in $\mathrm{H}-2 \mathrm{~Kb}^{10}$ and $\mathrm{H}-2 \mathrm{~Kb}^{\text {hi }}$ cell populations. Notch1-FL, full

720 length; -TM, transmembrane; -IC, intracellular. GAPDH is used as a loading control. (c) FACS

721 analyses of basal and IFN-y-induced (100 ng/mL for $24 \mathrm{hr}$.) surface H-2Kb expression (darker

722 colored histograms) in $\mathrm{H}-2 \mathrm{~Kb}^{10}$ and $\mathrm{H}-2 \mathrm{~Kb}^{\text {hi }}$ cells compared to isotype controls (lighter colored

723 histograms). Plots representative of 2 independent experiments. (d) Upper, Schematic of OVA

724 binding assay. Lower, FACS analysis of surface $\mathrm{H}-2 \mathrm{~Kb}-$ bound SIINFEKL OVA peptide in $\mathrm{H}-2 \mathrm{~Kb}^{\mathrm{hi}}$

725 and $\mathrm{H}-2 \mathrm{~Kb}^{10}$ cells under basal or IFN- $\mathrm{Y}$-induced conditions as in (c) and in the absence or

726 presence of the OVA peptide. Plots representative of 2 independent experiments. (e) Upper,

727 Schematic of NB-9464-OT-I co-culture assay. Lower, Contour plots showing the percentage of

728 naïve OT-I cells that were activated $\left(\mathrm{CD}^{+} \mathrm{CD}^{+} 9^{+}\right)$following co-culture with $\mathrm{H}-2 \mathrm{~Kb}^{\text {lo }}$ and $\mathrm{H}-2 \mathrm{~Kb}^{\mathrm{hi}}$

729 cells for $24 \mathrm{hr}$. with or without the OVA peptide. OT-I activation was measured by FACS analysis

730 of cell surface CD69. (f) Left, Immunohistochemical (IHC) staining for MYCN and CD8 expression

731 in representative murine NB xenograft tumors derived from NB-9464 H-2Kb'o (adrenergic) and H-

$7322 \mathrm{~Kb}^{\text {hi }}$ (mesenchymal) cells in immunocompetent syngeneic (C57BL/6) mice. Scale bars, $100 \mu \mathrm{m}$.

733 Right, Bar graphs showing the percentage of area occupied by CD8 ${ }^{+}$T cells in $\mathrm{H}-2 \mathrm{~Kb}^{10}(0.9 \% \pm$

$7340.2 \%)$ vs. $\mathrm{H}-2 \mathrm{~Kb}^{\text {hi }}(2.3 \% \pm 0.5 \%)$ tumors; ${ }^{\star} P<0.05$, two-tailed Welch's t-test. Each dot represents

735 one of three independent measurements for each tumor. Data represent mean \pm SEM. 
Sengupta et al.

\section{Supplementary Fig. 8.}

a

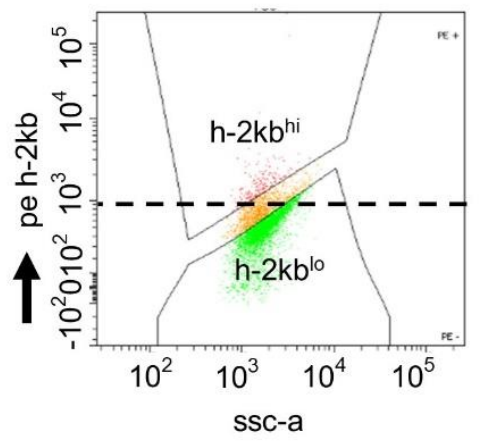

d

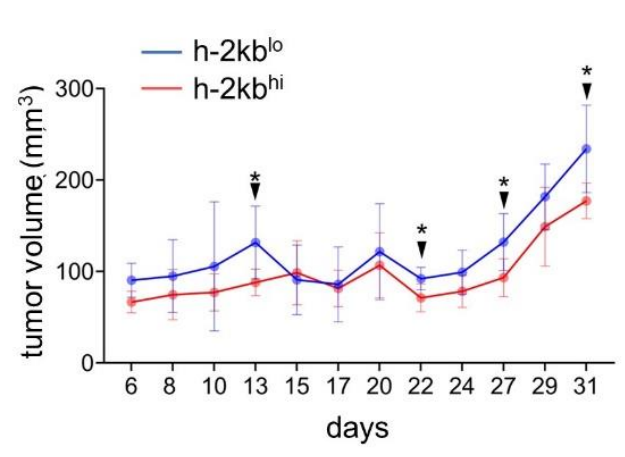

b

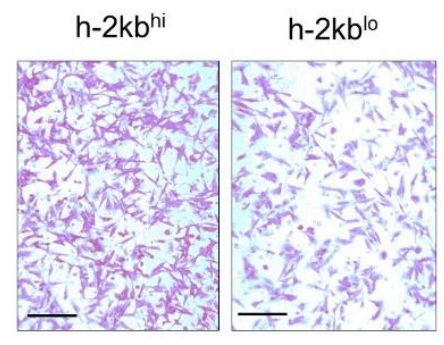

e

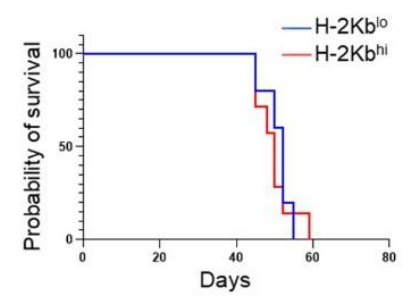

C
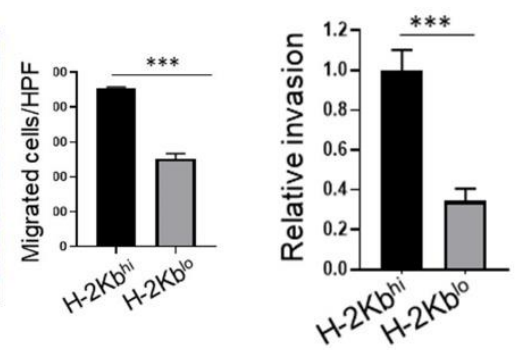

f

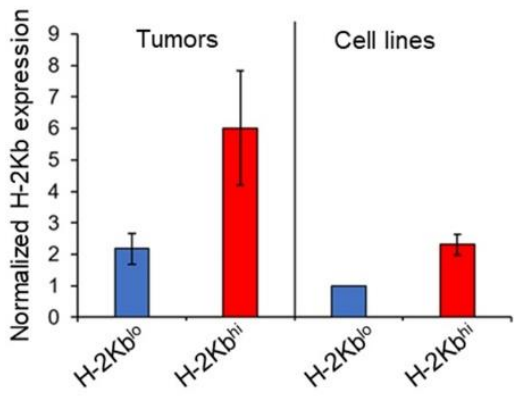

g

mesenchymal $\left(\mathrm{h}-2 \mathrm{~kb} \mathrm{~b}^{\mathrm{hi}}\right)$ tumor

adrenergic $\left(\mathrm{h}-2 \mathrm{~kb} b^{\mathrm{lo}}\right)$ tumor
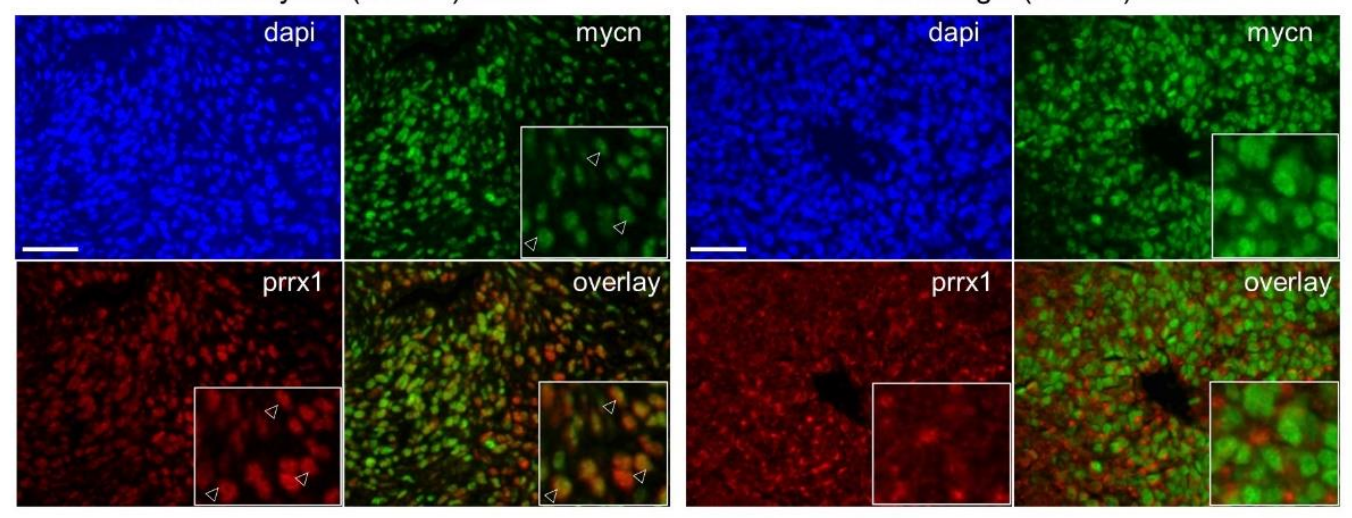
Sengupta et al. NB-9464 cells into $\mathrm{H}-2 \mathrm{~Kb}^{\text {hi }}$ and $\mathrm{H}-2 \mathrm{~Kb}^{10}$ populations. $\mathrm{X}$ axis represents side scatter (SSC-A); Y axis denotes fluorescence intensity of surface $\mathrm{H}-2 \mathrm{~Kb}$ detected using phycoerythrin (PE)conjugated antibody against $\mathrm{H}-2 \mathrm{~Kb}$. A logscale expression value of $10^{3}$ was used as the threshold

741 (horizontal dashed line) to gate $\mathrm{H}-2 \mathrm{~Kb}^{\text {hi }}\left(\geq 10^{3}\right)$ and $\mathrm{H}-2 \mathrm{~Kb}^{10}\left(<10^{3}\right)$ populations. (b) Left, Bright

742 field images of crystal violet-stained $\mathrm{H}-2 \mathrm{~Kb}^{\text {hi }}$ and $\mathrm{H}-2 \mathrm{~Kb}^{10}$ cells in transwell migration assays. Scale

743 bars, $100 \mu \mathrm{m}$. Right, Quantification of migrating cells per high-power field (HPF). Data represent

744 the means $\pm \mathrm{SD}, n=2$ biological replicates, ${ }^{\star \star \star} P<0.001$; two-tailed Student's t-test. (c)

745 Quantification of the relative invasiveness of $\mathrm{H}-2 \mathrm{~Kb}^{\text {hi }}$ and $\mathrm{H}-2 \mathrm{~Kb}^{10}$ cells. Data represent the means

$746 \pm \mathrm{SD}, n=2$ biological replicates, ${ }^{* *} P<0.001$; two-tailed Student's t-test. (d) Tumor volumes in

747 immunocompetent C57BL/6 mice injected subcutaneously with $1 \times 10^{6} \mathrm{H}-2 \mathrm{~Kb}^{10}$ or $\mathrm{H}-2 \mathrm{~Kb}^{\text {hi }} \mathrm{NB}$ -

7489464 cells. Measurements were started on day 3 after injection and continued three times weekly

749 for up to 50 days or until euthanized due to tumor growth. Graphs represent changes in tumor

750 volume until day 31 (means $\pm S D ; n=7$ per group at all time points) to highlight an earlier onset

751 of tumor formation with $\mathrm{H}-2 \mathrm{~Kb}^{\text {lo }}$ cells compared to $\mathrm{H}-2 \mathrm{~Kb}^{\text {hi }}$ cells. Tumors were considered to be

752 established upon reaching a volume of $\sim 250 \mathrm{~mm}^{3}$ (observed between days 31-34 for both $\mathrm{H}-2 \mathrm{~Kb}^{10}$

753 and $\mathrm{H}-2 \mathrm{~Kb}^{\text {hi }}$ tumors), following which both tumor types displayed equal increases in tumor growth

754 (data not shown). Tumor onset was defined as the day following which tumor volumes showed a

755 consistent increase $\left(24.3 \pm 2.2\right.$ days for $\mathrm{H}-2 \mathrm{~Kb}^{\mathrm{lo}}$ and $27.4 \pm 2.1$ days for $\mathrm{H}-2 \mathrm{~Kb}^{\mathrm{hi}}$ cells, $\left.P<0.05\right)$.

756 Closed arrows refer to the indicated days (13, 22, 27 and 31) on which there were significant

757 differences in tumor volumes (day $13, P=0.02$; day $22, P=0.01$; day $27, P=0.02$ and day 31 ,

$758 P=0.02 ; n=7$ per group at all time points). All $P$-values calculated using the two-tailed Student's

759 t-test. (e) Kaplan-Meier survival analysis of immunocompetent C57BL/6 mice bearing NB tumor

760 xenografts derived from $\mathrm{H}-2 \mathrm{~Kb}^{\mathrm{lo}}$ (adrenergic) and $\mathrm{H}-2 \mathrm{~Kb}^{\text {hi }}$ (mesenchymal) cells $(50.8 \pm 3.7$ vs.

$76149.8 \pm 4.8$ days; $P=0.7 ; \mathrm{n}=7$ per group; log-rank test. (f) $\mathrm{RT}$-qPCR analysis of $\mathrm{H}-2 \mathrm{~Kb}$ expression 
762 in $\mathrm{H}-2 \mathrm{~Kb}^{10}$ and $\mathrm{H}-2 \mathrm{~Kb}^{\text {hi }} \mathrm{NB}$ tumor xenografts and the cell lines used to generate the xenografts.

763 Data represent the means \pm SD, $n=3$ biological replicates. (g) Immunofluorescence images of

764 MYCN (green) and Prrx1 (red) expression in representative murine NB xenograft tumors derived

765 from NB-9464 H-2Kb ${ }^{\text {hi }}$ (mesenchymal) and $\mathrm{H}-2 \mathrm{~Kb}^{10}$ (adrenergic) cells in immunocompetent

766 syngeneic (C57BL/6) mice. Nuclei are counterstained with DAPI (blue). Insets depict cells with

767 nuclear co-staining of MYCN and Prrx1 (arrowheads) and are exclusively present in the $\mathrm{H}-2 \mathrm{~Kb}^{\text {hi }}$

768 mesenchymal tumor. Scale bars, $100 \mu \mathrm{m}$, insets $33.3 \mu \mathrm{m}$. 
Sengupta et al.

769

770

771 round blue cells (Fig. 6f). We next analyzed the immune status of these tumors, reasoning that

772 tumors arising from immunogenic $\mathrm{H}-2 \mathrm{~Kb}^{\text {hi }}$ cells would be infiltrated by $\mathrm{T}$ cells. Indeed, $\mathrm{H}-2 \mathrm{~Kb}^{\text {hi }}$

773 tumors showed significantly higher $\mathrm{CD}^{+} \mathrm{T}$ cell infiltration compared with $\mathrm{H}-2 \mathrm{~Kb} \mathrm{~b}^{\mathrm{lo}}$ tumors (Fig. 6f).

774 Taken together, these results suggest that the immunogenic traits of mesenchymal 775 neuroblastoma cells translate into the recruitment of cytotoxic $\mathrm{T}$ cells into the tumor 776 microenvironment.

777

778

779 We next analyzed the functional relevance of the increased expression of the NKG2D NK cell 780 receptor ligands (ULBP1-3, MICA, MICB) seen in mesenchymal neuroblastoma tumors 781 (Supplementary Fig. 4c; Fig. 4i). Since the interaction of the receptor with its cognate ligands 782 on target cells is the first step towards a cytotoxic response (Fig. 7a), we compared the ability of 783 adrenergic (parental SH-SY5Y) and mesenchymal (LDK-resistant SH-SY5Y and SH-EP) cells to 784 bind to the recombinant NKG2D receptor fusion protein in an in vitro binding assay. Both 785 mesenchymal cell types showed increased binding to the NKG2D receptor, in keeping with their 786 increased cell surface expression of the ULBP2/3/5/6, MICA and MICB ligands (Fig. 7b). NK cell 787 cytotoxicity is mediated by granzyme proteases and the pore-forming protein perforin, which in 788 resting cells are stored in secretory lysosomes or lytic granules marked by lysosome-associated 789 membrane protein-1 (LAMP-1 or CD107a $)^{55}$. Upon target recognition, NK cells undergo 790 degranulation, or exocytosis of the lytic granules, which is associated with relocation of the 791 CD107a antigen to the cell membrane. Using cell-surface CD107a as a specific marker of 792 degranulation, we measured degranulation of peripheral blood NK cells harvested from healthy 793 human donors in the presence of parental SH-SY5Y, LDK-resistant SH-SY5Y and SH-EP targets. 
Sengupta et al.

Fig. 7 .

a

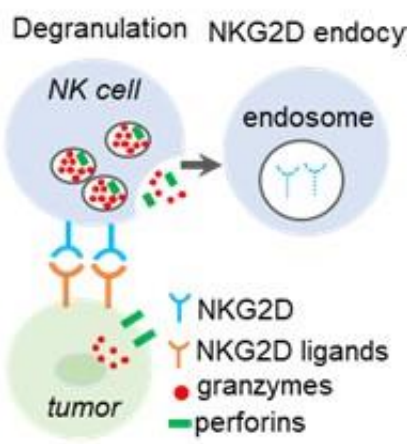

d $-5 Y$-par. $-5 Y$-LDK-res. - SH-EP

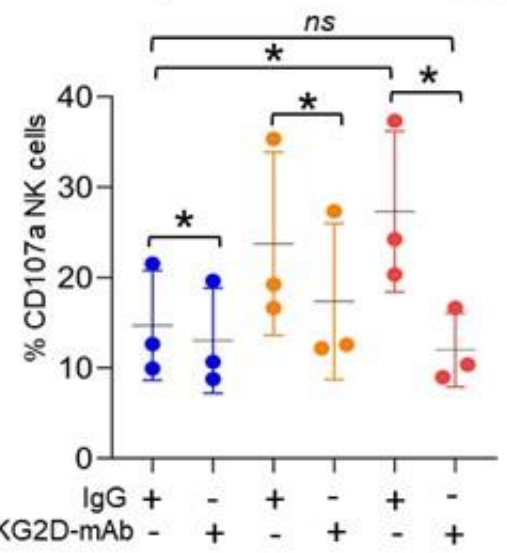

g

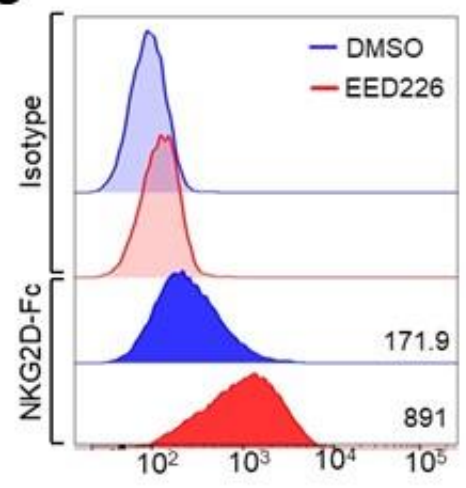

b

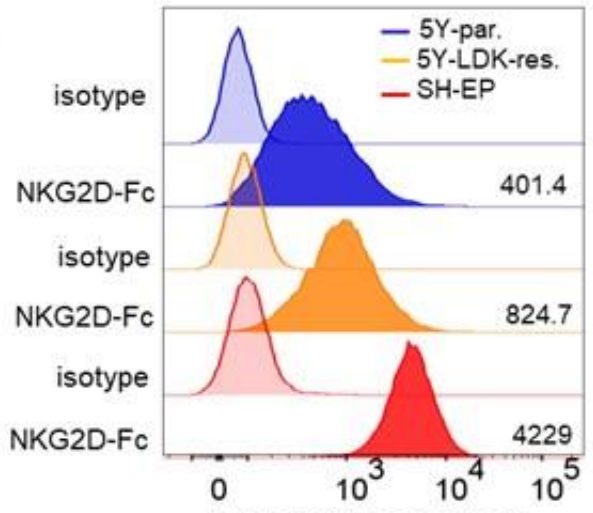

e Comp APC-A secondary

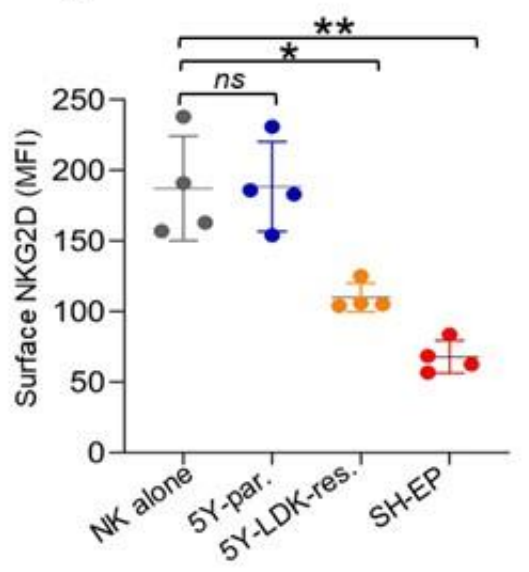

h

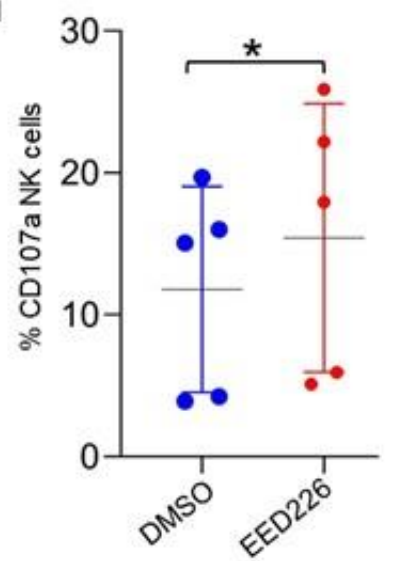

C
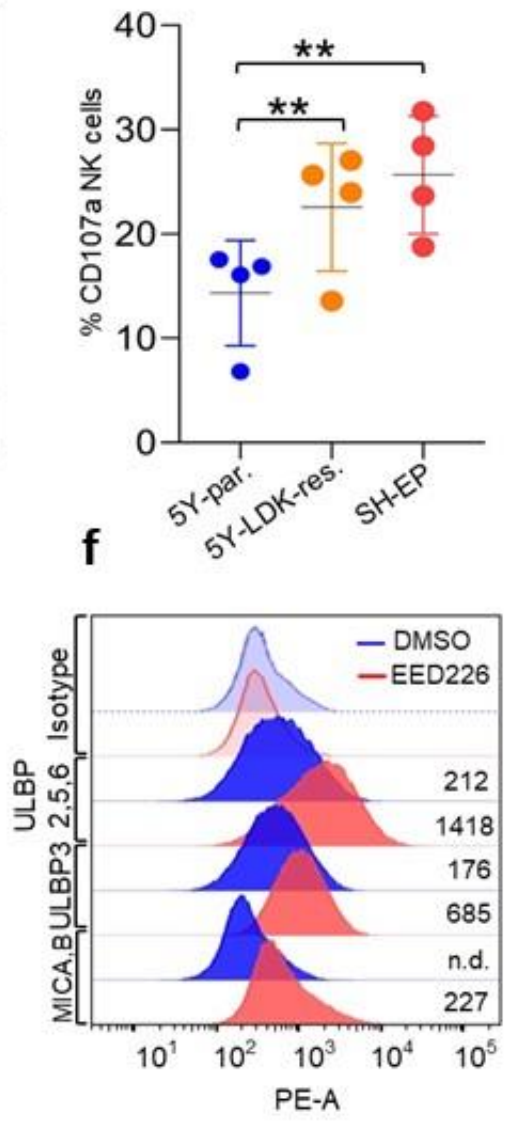

i

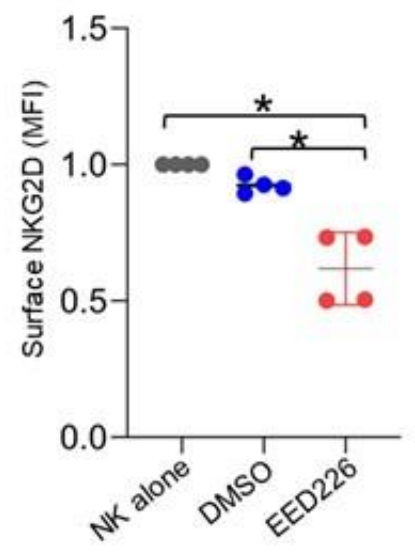


Sengupta et al.

794 Fig. 7. Mesenchymal NB cells induce NK cell degranulation. (a) Schematic representation of

795 the interaction between NKG2D receptors on NK cells and cognate ligands on tumor cells, leading

796 to NK cell degranulation and receptor endocytosis. (b) FACS analysis of purified human NKG2D-

797 Fc protein binding (darker histograms) to adrenergic parental SH-SY5Y, and mesenchymal LDK-

798 resistant SH-SY5Y and SH-EP cells. Comp Alexa-647, compensated fluorescence intensity of

799 NKG2D-Fc protein detected using Alexa 647-conjugated anti-human IgG. Lighter histograms

800 indicate staining with Alexa 647-conjugated anti-human IgG only. Numbers indicate median

801 fluorescence intensity (MFI). Plots representative of 2 independent experiments. (c) X-Y plot

802 showing the percentage of degranulating NK cells following co-culture with the same cells as in

803 (b) for $4 \mathrm{hr}$. at an effector: target (E: T) cell ratio of 1:2. Degranulation was measured by FACS analysis of cell-surface CD107a. Data represent the means \pm SD, $n=4$ biological replicates. (d)

805 X-Y plot showing the effect of a control IgG1 or an NKG2D blocking antibody on NK cell degranulation following co-culture with the indicated cells for $4 \mathrm{hr}$. NK cell degranulation was measured as in (c). Data represent the means $\pm \mathrm{SD}, n=4$ biological replicates. (e) X-Y plot depicting the MFI of NKG2D expression measured by FACS on naïve NK cells (NK alone) or following co-culture with parental SH-SY5Y, LDK-resistant SH-SY5Y and SH-EP cells for $4 \mathrm{~h}$. Data represent the means $\pm S D, n=4$ biological replicates. (f) FACS analysis of surface

811 ULBP2/5/6, ULBP3, and MICA/MICB in adrenergic parental SH-SY5Y cells treated with DMSO

812 (vehicle control) or EED226 $f 5 \mu \mathrm{M}$ for 8 days). (g) FACS analysis of purified human NKG2D-FC

813 protein binding to parental SH-SY5Y cells treated with either DMSO or EED226 as in (f). Light

814 gray histograms indicate human IgG1 isotype control. Numbers on the right represent MFI values

815 for FACS plots. Plots in (f) and (g) are representative of 2 independent experiments. (h, i) X-Y

816 plots showing NK cell degranulation (h) and MFI of surface-NKG2D (i) following co-culture of

817 naïve NK cells with parental SH-SY5Y cells treated with DMSO or EED226 as in (f). NK cell

818 granulation and NKG2D MFI were measured as in (c) and (e). Data represent means \pm SD, $n=$ 
bioRxiv preprint doi: https://doi.org/10.1101/2021.01.29.428154; this version posted January 30, 2021. The copyright holder for this preprint (which was not certified by peer review) is the author/funder. All rights reserved. No reuse allowed without permission.

Sengupta et al.

8194 (h) and 5 (i) biological replicates. Significance for all results was calculated using the paired

820 two-tailed Student's t-test $\left({ }^{\star} P<0.05 ;{ }^{* \star} P<0.01\right)$; ns, not significant 


\section{Supplementary Fig. 9.}

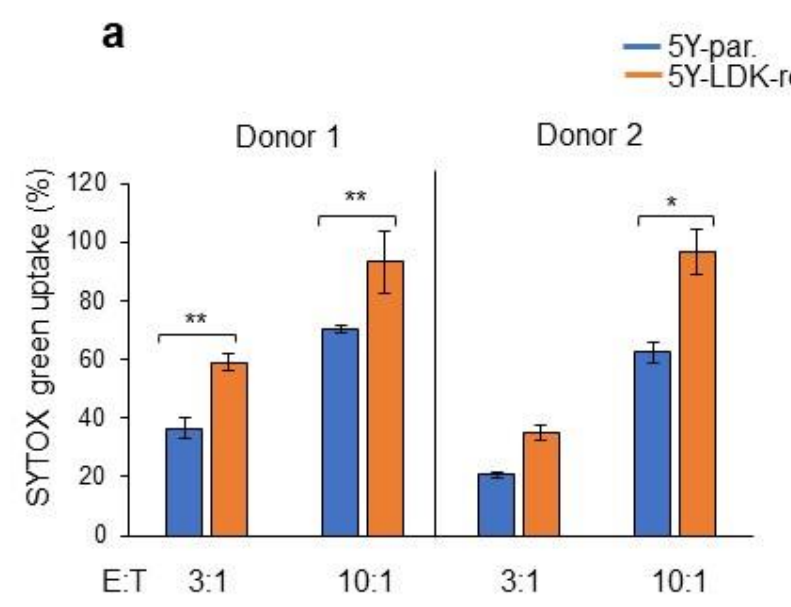

Supplementary Fig. 9. LDK-resistant SH-SY5Y cells are more susceptible to NK-induced cell death.

(a) Bargraphs showing NK-induced cell death in parental SH-SY5Y and LDK-resistant SH-SY5Y cells assessed by SYTOX green uptake following 1 hour of co-culture at indicated effector-to-target (E:T) ratios. Experiments were performed in two biological replicates using NK cells harvested from two independent donors. Data represent means $\pm S D, n=3$ technical replicates. Significance for all results was calculated using the paired two-tailed Student's t-test $\left({ }^{*} P<0.05 ;{ }^{*} \mathrm{P}<0.01\right)$. 
Sengupta et al.

821 Co-culture of NK cells with LDK-resistant SH-SY5Y and SH-EP cells, both of which express

822 ligands for the NKG2D receptor, resulted in increased NK cell degranulation compared to parental

823 SH-SY5Y cells that did not express these ligands (Fig. 7c). To confirm that the increased NK cell

824 degranulation in LDK-resistant SH-SY5Y and SH-EP cells was specific to the NKG2D receptor,

825 we blocked its function with an anti-NKG2D monoclonal antibody. Compared to the isotype

826 control, blockade of NKG2D receptor activity completely abrogated the increased NK cell

827 degranulation in LDK-resistant SH-SY5Y and SH-EP cells, but had no effects on parental SH-

828 SY5Y cells, signifying that the modest but robust increase in degranulation in the presence of

829 mesenchymal cells was specific to the NKG2D receptor on NK cells (Fig. 7d). Upon interaction

830 with their cognate ligands on target cells, NKG2D receptors are internalized via ubiquitin-

831 dependent endocytosis leading to their lysosomal degradation, rendering the loss of surface

832 NKG2D receptor expression a robust readout for ligand-receptor engagement ${ }^{56}$ (Fig. 7a). In line

833 with the presence of functional NKG2D ligands on mesenchymal cells, co-cultures with LDK-

834 resistant SH-SY5Y and SH-EP cells led to significant downregulation of NK cell surface-

835 associated NKG2D expression, whereas co-culture with the adrenergic parental SH-SY5Y cells

836 did not alter the abundance of surface NKG2D expression (Fig. 7e). Moreover, consistent with

837 the increased levels of NK degranulation upon co-culture with mesenchymal cells, LDK-resistant

838 SH-SY5Y were more susceptible to NK-induced cell death compared to parental SH-SY5Y cells

839 (Supplementary Fig. 9a)

The observation that genes encoding NKG2D ligands are repressed by the PRC2 complex in

842 adrenergic parental SH-SY5Y cells (Supplementary Fig. 7c) prompted us to examine whether

843 PRC2 inhibitors could induce the expression of these transcripts and influence NK cell function.

844 Indeed, treatment of parental SH-SY5Y cells with EED226, an allosteric inhibitor of the PRC2

845 complex ${ }^{57}$, led to increased expression and surface localization of ULBP2/3 and MICA/B NKG2D

846 ligands (Fig. 7f). Moreover, such increased ligand expression led to their increased binding to the 
847 NKG2D receptor fusion protein in EED226-treated cells compared to cells treated with DMSO

848 alone (Fig. 7g). Consequently, PRC2 inhibition resulted in an $\sim 20 \%$ increase in NK cell

849 degranulation (Fig. 7h). Finally, co-culture of primary NK cells with adrenergic parental SH-SY5Y

850 cells treated with EED226 led to a significant loss of surface NKG2D receptor expression (Fig.

851 7i), suggesting that the increased degranulation resulting from PRC2 inhibition was driven by the

852 NKG2D receptor. Overall, these results suggest that the lineage-specific expression of NK cell

853 ligands in mesenchymal neuroblastoma cells has a functional impact on NK cell activity, and that

854 pretreatment of adrenergic neuroblastoma cells could potentially render these cells susceptible

855 to NK-cell mediated immunotherapy by upregulating ligand expression. 
Sengupta et al.

\section{DISCUSSION}

857 Despite the relatively poor track record of immunotherapy for neuroblastoma, growing evidence

858 suggests that subsets of these tumors have the potential to induce a productive immune

859 response ${ }^{9}$. Here, we demonstrate that the mesenchymal cell state, characterized by neural crest

860 cell (NCC)-like phenotypes, is a strong predictor of an antitumor immune response in

861 neuroblastoma. Induction of this state was accompanied by the expression of tumor cell-intrinsic

862 immune response-inducing genes that were epigenetically repressed in the more differentiated

863 (adrenergic) tumor cells. Importantly, inhibition of the PRC2 complex relieved such repression of

864 ligands for the activating NK cell receptor NKG2D, and led to NK cell degranulation, suggesting

865 that this strategy could be explored as a potential measure to improve the response of patients

866 with adrenergic neuroblastomas to NK cell-mediated therapy.

867

We sought to identify neuroblastomas capable of eliciting an immune response as those characterized by the differential expression of immune gene signatures while remaining agnostic

870 to any of the established parameters that predict disease aggressiveness. Using UMAP

871 dimension reduction to analyze gene expression data from 498 primary neuroblastoma tumors,

872 we identified four clusters that were separated on the basis of differential activation of gene

873 networks that regulate the antitumor immune response, neuronal differentiation, MYCN-driven

874 processes and lipid metabolism. Intriguingly, the immunogenic cluster comprised almost equal

875 proportions of high- and low-risk tumors, raising the possibility that the molecular mechanisms

876 underlying immunogenicity in neuroblastoma are independent of disease aggressiveness. Thus,

877 our analysis using a cluster-based approach enabled the identification of antitumor immune

878 signatures as shared transcriptional programs between high- and low-risk neuroblastoma tumors,

879 a bridging feature that would have been missed in studies based solely on differential gene

880 expression between prognostically distinct groups of tumors. 
Sengupta et al.

A major finding of our study is the intimate link between tumor cell lineage and the propensity of eliciting an immune response. Neuroblastoma tumors show lineage plasticity, underscored by

884 a phenotypic switch between undifferentiated NCC-like and more differentiated cells, two

885 divergent cell states driven by distinct transcriptional programs ${ }^{37,38}$. Our analysis revealed that

886 neuroblastomas enriched in NCC-derived signatures showed significantly higher antitumor

887 immunity featuring T and NK cells compared to tumors enriched for signatures of the adrenergic

888 lineage. Our finding is substantiated by studies showing that diverse cellular states such as

889 stemness, senescence and metastasis strongly influence the engagement of innate and adaptive

890 immune pathways ${ }^{58-60}$. Importantly, while the NCC-like/mesenchymal state promoted immune

891 response mechanisms such as upregulation of $\mathrm{MHC}$ class I and infiltration of cytotoxic

892 lymphocytes, these tumors were also characterized by the activation of immune checkpoints such

893 as regulatory $\mathrm{T}$ cells and exhaustion markers linked to immune suppression, similar to those

894 observed in chronic virally infected states ${ }^{61}$. The presence of such seemingly contradictory gene

895 signatures has important therapeutic implications for selecting patients who are likely to benefit

896 from $T$ cell-based immunotherapies, as agents that target negative regulatory immune

897 checkpoints are likely to be most effective in those with a pre-existing but dampened antitumor

898 immune response ${ }^{35}$. Moreover, inhibition of cancer cell-intrinsic transcriptional programs that

899 promote T cell exclusion have been shown to effect changes in cell state and could potentially

900 employed to sensitize tumors to immunotherapy (Jerby-Arnon et al., 2018; Koyama et al., 2016;

901 Spranger et al., 2015).

902

903 In contrast to other MYC-driven cancers where high MYC levels restrain inflammatory 904 signaling and anti-tumor immune pathways ${ }^{41,62}$, tumors within our immunogenic cluster, enriched 905 largely for mesenchymal/NCC signatures, had relatively high MYC expression that positively 906 correlated with immune cell infiltration. These diametrically opposite roles of MYC in regulating 907 immune response suggest that its transcriptional functions are likely to be very different in cancers 
Sengupta et al.

908 in which aberrant MYC expression is the main oncogenic driver compared to MYCN-nonamplified

909 neuroblastomas that are not dependent on MYC overexpression. This notion is supported by the

910 observation that inhibiting endogenous MYC function in non MYC-driven pancreatic cancer

911 models leads to decreased recruitment and retention of inflammatory cells ${ }^{63}$. As MYC is an

912 essential TF in NCCs ${ }^{64,65}$, it is plausible that MYC promotes an immunogenic state by driving a

913 NCC-specific transcriptional program.

915 We also extended the analysis between cell state and immunogenicity to MYCN-amplified

916 tumors and identified a subset that incorporates both mesenchymal and immunogenic features.

917 This finding was substantiated by our data showing that expression of functional MHC class I is

918 retained in some $M Y C N$-amplified mesenchymal neuroblastoma cells and is not perturbed by

919 changes in MYCN levels. Indeed, a robust antitumor immune response is observed during the

920 early stages of tumor development in Th-MYCN mice and in mouse-human chimeric tumors

921 derived from human NCCs expressing $M Y C N$ and oncogenic $A L K^{17}$. Considering that these

922 mouse models are driven by gain of $M Y C N(\sim 4-8 \text { copies of the } M Y C N \text { transgene })^{17,52}$ rather than

923 the amplification seen in human tumors, and that absolute levels of MYCN protein dictate

924 transcriptional output ${ }^{66}$, the relatively low MYCN dosage in these tumors may account for their

925 immunogenicity. These findings, if confirmed in additional data sets, should encourage us to

926 reconsider the notion that all $M Y C N$-amplified tumors are intrinsically immune tolerant and that a

927 subset may in fact, be capable of inducing an immune response by virtue of their cell state.

929 The mutual exclusivity of the neuronal and immunogenic clusters suggests that 930 neuroblastomas with characteristics of neuronal differentiation (i.e. adrenergic phenotype) are 931 weakly immunogenic and thus incapable of inducing an effective immune response. Moreover,

932 the presence of NCC-derived gene expression signatures among immunogenic tumors indicates 933 that phenotypic reversal of sympathetic neuronal cells to an NCC-like state could contribute 
Sengupta et al.

934 significantly to the antitumor immune response. Such cell state-dependent immunogenic

935 switching could be mediated by the lineage-specific core regulatory circuitry (CRC) that drives

936 distinct transcriptomic states in neuroblastoma. Indeed, several TFs that constitute the NCC-like

937 (mesenchymal) CRC, including interferon regulatory factors 1-3 and IFI16, function as major

938 drivers of tumor cell-intrinsic innate and adaptive immune responses ${ }^{67,68}$. Furthermore, our data

939 identify PRRX1, another component of the mesenchymal CRC, as a regulator of MHC class I and

940 antigen-processing gene expression in neuroblastoma. Interestingly, PRRX1 was not identified

941 as a candidate regulator of MHC-I expression in genome-wide CRISPR knock-out screens to

942 identify NF-kB-dependent MHC-I suppressors in neuroblastoma ${ }^{69}$, suggesting that the

943 mechanisms employed by PRRX1 could be independent of NF-kB activation and may involve

944 direct transcriptional activation of these genes. In addition, the upregulation of DNA damage

945 sensor proteins such as IFI16 and STING in mesenchymal neuroblastomas could also contribute

946 to the increased tumor-infiltrating lymphocyte abundance in these tumors, as suggested by results

947 in small cell lung cancer ${ }^{70}$.

949 We have also established that the changes in immune gene expression accompanying the 950 adrenergic to mesenchymal transition are epigenetically regulated. Unlike lineage identity genes 951 that are regulated by super-enhancers, tumor cell-intrinsic immune genes involved in diverse 952 immune functions such as the inflammatory response, IFN-y signaling and NK cell recognition, 953 are governed through changes in promoter structure, achieved by either de novo acquisition of 954 permissive chromatin or an epigenetic switch from PRC2-mediated repression to a permissive 955 chromatin landscape. Our findings support a role for the PRC2 complex in repressing genes that 956 encode ligands for the activating NK cell receptor NKG2D in adrenergic neuroblastoma cells and

957 the use of PRC2 inhibitors to augment the NK cell response against these cells. This approach is 958 justified on several grounds: our results add to the growing body of evidence for tumor-cell 959 autonomous function of PRC2 as a barrier to anti-tumor immunity, achieved through inhibition of 
960 processes such as MHC expression ${ }^{11,71}$, antigen processing and presentation and inflammatory

961 cytokine production ${ }^{72,73}$. Moreover, our observation that NKG2D ligands are enriched in

962 mesenchymal neuroblastomas coupled with the demonstration of the critical effector role of NK

963 cells in the antitumor immune response against this tumor ${ }^{51}$ and the promising responses of

964 patients with other solid tumors to NKG2D-directed CAR NK cell therapy ${ }^{74}$ strengthen this

965 premise. Considered together, the results of our analysis identify cell lineage as an important

966 determinant of the immune responsiveness of neuroblastoma and suggest rationales for the use

967 of immune-based therapies, either alone or in combination with epigenetic inhibitors, against the

968 two divergent phenotypes that define the lineage state of this pediatric tumor. 
Sengupta et al.

971

972

973

974

975

976

977

978

979

980

981

982

983

984

985

986

987

988

989

990

991

992

993

994

995

996

997

998

999

1000

1001

1002

1003

1004

1005

\section{Acknowledgements}

We thank Matthew Harlow from the George lab, Sumit Sen Santara, Ying Zhang, and Zhibin Zhang from the Lieberman lab for helpful discussions. We thank Mark Zimmerman, A. Thomas Look and Kimberly Stegmaier for sharing cell lines. We are thankful to the following members of the former Haining lab at DFCI for sharing resources and experimental advice: Ulrike Gerdemann, Dawn Comstock, Kathleen Yates, Anna Word, and Adrienne Long. The results shown here are in part based on data curated by the R2: Genomics Analysis and Visualization Platform: http://r2.amc.nl/. This work was supported by a St. Baldrick's Foundation Childhood Cancer Research Grant (R.E.G. and R.J.) DOD CA191000 (R.E.G. and R.J.) and NIH grants R01CA197336; R01-CA148688 (R.E.G). St. S. is a recipient of the Pew Stewart Scholarship. Sa. S. and M.K. were supported by the Rally Foundation for Childhood Cancer Research and Infinite Love for Kids Fighting Cancer, B.C.M. by the National Center for Advancing Translational Sciences/NIH Award KL2 TR002542 and D.N.D. by an Alex's Lemonade Stand Foundation Young Investigator Fellowship. This manuscript is dedicated to the memory of John R. Gilbert, Scientific Editor.

\section{Author contributions}

Sa.S. and R.E.G. conceived the study. Sa.S., A.C., B.C.M., J.L., and R.E.G. designed the experiments. Sa.S. performed the molecular, cellular and genomic studies. S.D. conceived and performed the genomic and computational analysis with inputs from R.D., Sa.S. and R.E.G. Sa.S., A.C., and B.C.M. performed the T and NK cell studies. B.S. performed the animal and cloning experiments. S.Z. performed quantitative analysis of IHC images. H.H. performed the cell migration assays. M.K., D.N.D., and L.S. contributed to FACS analysis, generation of LDK378resistant SH-SY5Y cells, and compound testing, respectively. M.C., R.V., R.J., and St.S. contributed ideas towards regulation of immune function and cell lineage state. Sa.S., S.D., and R.E.G. wrote the manuscript with input from all authors.

\section{Competing Interests}

R.J. is a cofounder of Fate Therapeutics, Fulcrum Therapeutics, and Omega Therapeutics and an advisor to Dewpoint Therapeutics. 
Sengupta et al.

\section{References}

1007 1. Yu, A.L. et al. Anti-GD2 antibody with GM-CSF, interleukin-2, and isotretinoin for neuroblastoma. N Engl J Med 363, 1324-34 (2010). Cheung, N.K. \& Dyer, M.A. Neuroblastoma: developmental biology, cancer genomics and immunotherapy. Nat Rev Cancer 13, 397-411 (2013).

1012 Singh, N. et al. T cells targeting NY-ESO-1 demonstrate efficacy against disseminated neuroblastoma. Oncoimmunology 5, e1040216 (2016). lymphocyte clones in patients with neuroblastoma. Mol Ther 15, 825-33 (2007).

5. Pule, M.A. et al. Virus-specific T cells engineered to coexpress tumor-specific receptors: persistence and antitumor activity in individuals with neuroblastoma. Nat Med 14, 126470 (2008).

6. Louis, C.U. et al. Antitumor activity and long-term fate of chimeric antigen receptorpositive T cells in patients with neuroblastoma. Blood 118, 6050-6 (2011).

7. Merchant, M.S. et al. Phase I Clinical Trial of Ipilimumab in Pediatric Patients with Advanced Solid Tumors. Clin Cancer Res 22, 1364-70 (2016).

1023

1024

1025

8. Davis, K.L. et al. Nivolumab in children and young adults with relapsed or refractory solid tumours or lymphoma (ADVL1412): a multicentre, open-label, single-arm, phase 1-2 trial. Lancet Oncol 21, 541-550 (2020).

9. Richards, R.M., Sotillo, E. \& Majzner, R.G. CAR T Cell Therapy for Neuroblastoma. Front Immunol 9, 2380 (2018).

1027

10. Bernards, R., Dessain, S.K. \& Weinberg, R.A. N-myc amplification causes downmodulation of MHC class I antigen expression in neuroblastoma. Cell 47, 667-74 (1986).

11. Burr, M.L. et al. An Evolutionarily Conserved Function of Polycomb Silences the MHC Class

1031

1032 I Antigen Presentation Pathway and Enables Immune Evasion in Cancer. Cancer Cell 36, 385-401 e8 (2019).

1033

12. Raffaghello, L. et al. Multiple defects of the antigen-processing machinery components in human neuroblastoma: immunotherapeutic implications. Oncogene 24, 4634-44 (2005).

1034

1035

1036

13. Raffaghello, L. et al. Downregulation and/or release of NKG2D ligands as immune evasion strategy of human neuroblastoma. Neoplasia 6, 558-68 (2004).

1037

1038

1039

14. Castriconi, R. et al. Identification of 4lg-B7-H3 as a neuroblastoma-associated molecule that exerts a protective role from an NK cell-mediated lysis. Proc Natl Acad Sci U S A 101, 12640-5 (2004).

1040

1041

1042

1043

1044

1045

1046

1047

15. Coughlin, C.M. et al. Immunosurveillance and survivin-specific T-cell immunity in children with high-risk neuroblastoma. J Clin Oncol 24, 5725-34 (2006).

16. Betancur, P.A. et al. A CD47-associated super-enhancer links pro-inflammatory signalling to CD47 upregulation in breast cancer. Nat Commun 8, 14802 (2017).

17. Cohen, M.A. et al. Formation of Human Neuroblastoma in Mouse-Human Neural Crest Chimeras. Cell Stem Cell 26, 579-592 e6 (2020).

18. Asgharzadeh, S. et al. Clinical significance of tumor-associated inflammatory cells in metastatic neuroblastoma. J Clin Oncol 30, 3525-32 (2012).

1048

19. Song, L. et al. Valpha24-invariant NKT cells mediate antitumor activity via killing of tumorassociated macrophages. J Clin Invest 119, 1524-36 (2009). 
Sengupta et al.

1049

1050

1051

1052

1053

1054

1055

1056

1057

1058

1059

1060

1061

1062

1063

1064

1065

1066

1067

1068

1069

1070

1071

1072

1073

1074

1075

1076

1077

1078

1079

1080

1081

1082

1083

1084

1085

1086

1087

1088

1089

1090

1091

1092

20. Mao, Y. et al. Targeting Suppressive Myeloid Cells Potentiates Checkpoint Inhibitors to Control Spontaneous Neuroblastoma. Clin Cancer Res 22, 3849-59 (2016).

21. Tran, H.C. et al. TGFbetaR1 Blockade with Galunisertib (LY2157299) Enhances AntiNeuroblastoma Activity of the Anti-GD2 Antibody Dinutuximab (ch14.18) with Natural Killer Cells. Clin Cancer Res 23, 804-813 (2017).

22. Brodeur, G.M., Seeger, R.C., Schwab, M., Varmus, H.E. \& Bishop, J.M. Amplification of Nmyc in untreated human neuroblastomas correlates with advanced disease stage. Science 224, 1121-4 (1984).

23. Seeger, R.C. et al. Association of multiple copies of the N-myc oncogene with rapid progression of neuroblastomas. N Engl J Med 313, 1111-6 (1985).

24. Layer, J.P. et al. Amplification of N-Myc is associated with a T-cell-poor microenvironment in metastatic neuroblastoma restraining interferon pathway activity and chemokine expression. Oncoimmunology 6, e1320626 (2017).

25. Wei, J.S. et al. Clinically Relevant Cytotoxic Immune Cell Signatures and Clonal Expansion of T-Cell Receptors in High-Risk MYCN-Not-Amplified Human Neuroblastoma. Clin Cancer Res 24, 5673-5684 (2018).

26. Brandetti, E. et al. MYCN is an immunosuppressive oncogene dampening the expression of ligands for NK-cell-activating receptors in human high-risk neuroblastoma. Oncoimmunology 6, e1316439 (2017).

27. Valentijn, L.J. et al. Functional MYCN signature predicts outcome of neuroblastoma irrespective of MYCN amplification. Proc Natl Acad Sci U S A 109, 19190-5 (2012).

28. Durbin, B.P., Hardin, J.S., Hawkins, D.M. \& Rocke, D.M. A variance-stabilizing transformation for gene-expression microarray data. Bioinformatics 18 Suppl 1, S105-10 (2002).

29. Lin, S.M., Du, P., Huber, W. \& Kibbe, W.A. Model-based variance-stabilizing transformation for Illumina microarray data. Nucleic Acids Res 36, e11 (2008).

30. McInnes L, H.J. UMAP: Uniform manifold approximation and projection for dimension reduction. arXiv preprint arXiv:1802.03426. (2018).

31. Becht, E. et al. Dimensionality reduction for visualizing single-cell data using UMAP. Nat Biotechnol (2018).

32. Brodeur, G.M. et al. Revisions of the international criteria for neuroblastoma diagnosis, staging, and response to treatment. Journal of Clinical Oncology 11, 1466-1477 (1993).

33. Cohn, S.L. et al. The International Neuroblastoma Risk Group (INRG) classification system: an INRG Task Force report. J Clin Oncol 27, 289-97 (2009).

34. Satija, R., Farrell, J.A., Gennert, D., Schier, A.F. \& Regev, A. Spatial reconstruction of singlecell gene expression data. Nat Biotechnol 33, 495-502 (2015).

35. Spranger, S. et al. Up-regulation of PD-L1, IDO, and T(regs) in the melanoma tumor microenvironment is driven by CD8(+) T cells. Sci Transl Med 5, 200ra116 (2013).

36. Wherry, E.J. \& Kurachi, M. Molecular and cellular insights into T cell exhaustion. Nat Rev Immunol 15, 486-99 (2015).

37. Boeva, V. et al. Heterogeneity of neuroblastoma cell identity defined by transcriptional circuitries. Nat Genet 49, 1408-1413 (2017).

38. van Groningen, T. et al. Neuroblastoma is composed of two super-enhancer-associated differentiation states. Nat Genet 49, 1261-1266 (2017). 
Sengupta et al.

1093

1094

1095

1096

1097

1098

1099

1100

1101

1102

1103

1104

1105

1106

1107

1108

1109

1110

1111

1112

1113

1114

1115

1116

1117

1118

1119

1120

1121

1122

1123

1124

1125

1126

1127

1128

1129

1130

1131

1132

1133

1134

1135

1136

39. Bindea, G. et al. Spatiotemporal dynamics of intratumoral immune cells reveal the immune landscape in human cancer. Immunity 39, 782-95 (2013).

40. Wang, L.L. et al. Augmented expression of MYC and/or MYCN protein defines highly aggressive MYC-driven neuroblastoma: a Children's Oncology Group study. Br J Cancer 113, 57-63 (2015).

41. Casey, S.C. et al. MYC regulates the antitumor immune response through CD47 and PDL1. Science 352, 227-31 (2016).

42. Cursons, J. et al. A Gene Signature Predicting Natural Killer Cell Infiltration and Improved Survival in Melanoma Patients. Cancer Immunol Res 7, 1162-1174 (2019).

43. Newman, A.M. et al. Robust enumeration of cell subsets from tissue expression profiles. Nat Methods 12, 453-7 (2015).

44. $\mathrm{Hu}, \mathrm{X}$. et al. Landscape of B cell immunity and related immune evasion in human cancers. Nat Genet 51, 560-567 (2019).

45. Ross, R.A., Spengler, B.A. \& Biedler, J.L. Coordinate morphological and biochemical interconversion of human neuroblastoma cells. J Natl Cancer Inst 71, 741-7 (1983).

46. Cohn, S.L. et al. Prolonged N-myc protein half-life in a neuroblastoma cell line lacking Nmyc amplification. Oncogene 5, 1821-7 (1990).

47. Debruyne, D.N. et al. ALK inhibitor resistance in ALK(F1174L)-driven neuroblastoma is associated with AXL activation and induction of EMT. Oncogene 35, 3681-91 (2016).

48. Chipumuro, E. et al. CDK7 inhibition suppresses super-enhancer-linked oncogenic transcription in MYCN-driven cancer. Cell 159, 1126-1139 (2014).

49. Durbin, A.D. et al. Selective gene dependencies in MYCN-amplified neuroblastoma include the core transcriptional regulatory circuitry. Nat Genet 50, 1240-1246 (2018).

50. Margueron, R. \& Reinberg, D. The Polycomb complex PRC2 and its mark in life. Nature 469, 343-9 (2011).

51. Kroesen, M. et al. A transplantable TH-MYCN transgenic tumor model in C57BI/6 mice for preclinical immunological studies in neuroblastoma. Int J Cancer 134, 1335-45 (2014).

52. Weiss, W.A., Aldape, K., Mohapatra, G., Feuerstein, B.G. \& Bishop, J.M. Targeted expression of MYCN causes neuroblastoma in transgenic mice. EMBO J 16, 2985-95 (1997).

53. Clarke, S.R. et al. Characterization of the ovalbumin-specific TCR transgenic line OT-I: MHC elements for positive and negative selection. Immunol Cell Biol 78, 110-7 (2000).

54. Cibrian, D. \& Sanchez-Madrid, F. CD69: from activation marker to metabolic gatekeeper. Eur J Immunol 47, 946-953 (2017).

55. Uhrberg, M. The CD107 mobilization assay: viable isolation and immunotherapeutic potential of tumor-cytolytic NK cells. Leukemia 19, 707-9 (2005).

56. Molfetta, R. et al. Regulation of NKG2D Expression and Signaling by Endocytosis. Trends Immunol 37, 790-802 (2016).

57. Qi, W. et al. An allosteric PRC2 inhibitor targeting the H3K27me3 binding pocket of EED. Nat Chem Biol 13, 381-388 (2017).

58. Dongre, A. \& Weinberg, R.A. New insights into the mechanisms of epithelial-mesenchymal transition and implications for cancer. Nat Rev Mol Cell Biol 20, 69-84 (2019).

59. Malladi, S. et al. Metastatic Latency and Immune Evasion through Autocrine Inhibition of WNT. Cell 165, 45-60 (2016). 
Sengupta et al.

1137 60. Pereira, B.I. et al. Senescent cells evade immune clearance via HLA-E-mediated NK and CD8(+) T cell inhibition. Nat Commun 10, 2387 (2019).

61. Shin, H. \& Wherry, E.J. CD8 T cell dysfunction during chronic viral infection. Curr Opin Immunol 19, 408-15 (2007).

62. Kortlever, R.M. et al. Myc Cooperates with Ras by Programming Inflammation and Immune Suppression. Cell 171, 1301-1315 e14 (2017).

63. Sodir, N.M. et al. Endogenous Myc maintains the tumor microenvironment. Genes Dev 25, 907-16 (2011).

64. Bellmeyer, A., Krase, J., Lindgren, J. \& LaBonne, C. The protooncogene c-myc is an essential regulator of neural crest formation in xenopus. Dev Cell 4, 827-39 (2003).

65. Rada-Iglesias, A. et al. Epigenomic annotation of enhancers predicts transcriptional regulators of human neural crest. Cell Stem Cell 11, 633-48 (2012).

66. Zeid, R. et al. Enhancer invasion shapes MYCN-dependent transcriptional amplification in neuroblastoma. Nat Genet 50, 515-523 (2018).

67. Chang, C.H., Hammer, J., Loh, J.E., Fodor, W.L. \& Flavell, R.A. The activation of major histocompatibility complex class I genes by interferon regulatory factor-1 (IRF-1). Immunogenetics 35, 378-84 (1992).

68. Unterholzner, L. et al. IFI16 is an innate immune sensor for intracellular DNA. Nat Immunol 11, 997-1004 (2010).

69. Spel, L. et al. Nedd4-Binding Protein 1 and TNFAIP3-Interacting Protein 1 Control MHC-1 Display in Neuroblastoma. Cancer Res 78, 6621-6631 (2018).

70. Sen, T. et al. Targeting DNA Damage Response Promotes Antitumor Immunity through STING-Mediated T-cell Activation in Small Cell Lung Cancer. Cancer Discov 9, 646-661 (2019).

71. Ennishi, D. et al. Molecular and Genetic Characterization of MHC Deficiency Identifies EZH2 as Therapeutic Target for Enhancing Immune Recognition. Cancer Discov 9, 546-563 (2019).

72. Zingg, D. et al. The Histone Methyltransferase Ezh2 Controls Mechanisms of Adaptive Resistance to Tumor Immunotherapy. Cell Rep 20, 854-867 (2017).

73. Peng, D. et al. Epigenetic silencing of TH1-type chemokines shapes tumour immunity and immunotherapy. Nature 527, 249-53 (2015).

74. Xiao, L. et al. Adoptive Transfer of NKG2D CAR mRNA-Engineered Natural Killer Cells in Colorectal Cancer Patients. Mol Ther 27, 1114-1125 (2019).

75. Schindelin, J. et al. Fiji: an open-source platform for biological-image analysis. Nat Methods 9, 676-82 (2012).

76. Kamentsky, L. et al. Improved structure, function and compatibility for CellProfiler: modular high-throughput image analysis software. Bioinformatics 27, 1179-80 (2011).

77. Love, M.I., Huber, W. \& Anders, S. Moderated estimation of fold change and dispersion for RNA-seq data with DESeq2. Genome Biol 15, 550 (2014).

78. Zhu, X. et al. Single-Cell Clustering Based on Shared Nearest Neighbor and Graph Partitioning. Interdiscip Sci 12, 117-130 (2020).

79. Russo, P.S.T. et al. CEMiTool: a Bioconductor package for performing comprehensive modular co-expression analyses. BMC Bioinformatics 19, 56 (2018). 
1180 80. Yu, G., Wang, L.G., Han, Y. \& He, Q.Y. clusterProfiler: an R package for comparing biological

1181

1182

1183

1184

1185

1186

1187

1188

1189 themes among gene clusters. OMICS 16, 284-7 (2012).

81. Alexopoulos, E.C. Introduction to multivariate regression analysis. Hippokratia 14, 23-8 (2010).

82. Das, S. \& Bansal, M. Variation of gene expression in plants is influenced by gene architecture and structural properties of promoters. PLoS One 14, e0212678 (2019).

83. Debruyne, D.N. et al. BORIS promotes chromatin regulatory interactions in treatmentresistant cancer cells. Nature 572, 676-680 (2019). 
Sengupta et al.

\section{Methods}

\section{Cell culture}

1192 Human neuroblastoma (NB) cell lines (Kelly, NBL-S, CHP-212, SH-SY5Y, SH-EP, CHLA-20, 1193 NB69, SK-N-FI) were obtained from the Children's Oncology Group cell line bank. ACN, GI-ME1194 N, NB-EbC1 were kind gifts from A. Thomas Look and Kimberly Stegmaier at Dana Farber Cancer 1195 Institute (DFCl). NB-9464 cells were provided by To-Ha Thai at Beth Israel Deaconess Medical 1196 Center, Boston, MA. The cell lines were authenticated through STR analyses at the DFCl Core 1197 facility and were routinely tested for mycoplasma. All NB cells were grown in RPMI-1640 medium 1198 (Invitrogen) supplemented with 10\% fetal bovine serum (FBS) (Invitrogen) and 1\% 1199 penicillin/streptomycin (Life Technologies). HEK293T cells obtained from the American Type 1200 Culture Collection (ATCC) were grown in DMEM (Invitrogen) supplemented with 10\% FBS and 1201 1\% penicillin/streptomycin (Life Technologies). SH-SY5Y cells resistant to the ALK inhibitor 1202 ceritinib (LDK378) were described previously (Debruyne et al., 2016) and were grown in complete 1203 RPMI-1640 in the presence of $1.5 \mu \mathrm{M}$ ceritinib.

\section{Generation of PRRX1-inducible cell lines}

1206 Lentiviral vectors containing wild type and DNA-binding mutants of PRRX1 were generated by 1207 cloning cDNAs encoding full length or homeodomain deletions of the human PRRX1A sequence 1208 into the plnducer20 lentiviral plasmid (gift from Stephen Elledge, Addgene plasmid \#44012). The 1209 DNA-binding mutants harbor individual deletions of the three $\alpha$-helices $(\Delta \mathrm{H} 1, \Delta \mathrm{H} 2$ and $\Delta \mathrm{H} 3)$ within 1210 the PRRX1 homeodomain (amino acids (aa) 94-153). Amino acid boundaries of the deleted 1211 regions are as follows: $\Delta H 1$ (aa 103-116); $\Delta H 2$ (aa 121-131); $\Delta H 3$ (aa 135-151). The lentivirus 1212 was packaged by co-transfection of plnducer20 plasmid with the helper plasmids, pCMV1213 deltaR8.91 and pMD2.G-VSV-G into HEK293T cells using the TransIT-LT1 Transfection Reagent 1214 (Mirus Bio LLC). Virus-containing supernatants were collected $48 \mathrm{hr}$ after transfection. SH-SY5Y 1215 cells were transduced with the viral supernatant in the presence of $8 \mu \mathrm{g} / \mathrm{ml}$ polybrene (Sigma- 
Sengupta et al.

1216

1217

1218

1219

1220

1221

1222

1223

1224

1225

1226

1227

1228

1229

1230

1231 FACS analysis for cell surface protein staining

1232 For each staining reaction $1 \times 10^{6}$ live cells were placed in a $12 \times 75 \mathrm{~mm}$ polystyrene round bottom

1233 tube (Falcon), resuspended in $100 \mu \mathrm{l}$ 1x PBS and stained with the Zombie near-infrared (Zombie

1234 NIR) viability dye (BioLegend) at a 1:1,000 dilution for 15 minutes at RT. Cells were then washed

1235 once in FACS buffer (0.5\% BSA in 1x PBS), resuspended in $100 \mu$ l of FACS buffer and incubated

1236 in $5 \mu$ l of Human TruStain FcX ${ }^{\mathrm{TM}}$ (Fc receptor blocking solution, BioLegend) for 10 minutes at RT.

1237 Next, appropriate volumes of conjugated fluorescent primary antibodies at predetermined 1238 optimum concentrations were added and incubated on ice for 20 minutes in the dark. Cells were

1239 then washed once in $2 \mathrm{ml}$ of FACS buffer by centrifugation at 1500 rpm for 5 minutes. All FACS

1240 samples were analyzed on a FACSCalibur flow cytometer (Becton Dickinson) using Cell Quest

1241 software (Becton Dickinson). A minimum of 50,000 events was counted per sample and used for 
Sengupta et al.

1242 further analysis. Data were analyzed using FlowJo v10 software (Becton Dickinson). The following

1243 primary antibodies were used: PE-HLA (Biolegend; clone W6/32), PE-MICA/B (Biolegend clone

1244 6D4), PE-ULBP2 (R\&D Systems; clone 165903), PE-ULBP3 (R\&D Systems; clone 166510), PE-

$1245 \mathrm{H}-2 \mathrm{~Kb}$ (Biolegend AF6-88.5), PE-H-2Kb SIINFEKL (Biolegend; clone 25-D1.16), PE-mouse

1246 IgG2a k isotype control (Biolegend MOPC-173), PE- mouse lgG1 k isotype control (MOPC-21).

\section{Cell viability assay}

1249 SH-SY5Y parental and SH-SY5Y LDK-resistant cells were seeded in 96-well plates at a density 1250 of $2 \times 10^{3}$ cells/well. After $24 \mathrm{~h}$, cells were treated with increasing concentrations of LDK378 1251 (ranging from $1 \mathrm{nM}$ to $10 \mu \mathrm{M}$ ) dissolved in Dimethyl Sulfoxide (DMSO). DMSO solvent without the 1252 drug served as a negative control. Following $72 \mathrm{~h}$ incubation, cells were analyzed for viability

1253 using the CellTiter-Glo Luminescent Cell Viability Assay (Promega) according to the 1254 manufacturer's instructions. Drug concentrations that inhibited cell growth by $50 \%\left(\mathrm{IC}_{50}\right)$ were 1255 determined using a non-linear regression curve fit with GraphPad Prism 8 software.

\section{Cell migration and invasion assays}

1258 Cell migration was measured using transwell chambers (Falcon). NB9464-H-2Kb ${ }^{10}$ or 1259 NB9464-H-2Kb ${ }^{\text {hi }}$ cells in serum-free medium $\left(0.5 \times 10^{6}\right.$ cells $\left./ \mathrm{ml}\right)$ were added to the upper 1260 chamber and inserts ( $8 \mu \mathrm{m}$ pore size) were placed in the lower chamber containing medium 1261 with $10 \%$ FBS. Following incubation at $37^{\circ} \mathrm{C}$ for $8 \mathrm{~h}$, cells that migrated to the lower chamber 1262 were fixed with methanol and stained with crystal violet (Sigma-Aldrich). The stained cells 1263 were photographed with a light microscope at 100X magnification and migration was 1264 quantified as the number of cells per high power field. Cell invasion was measured using the

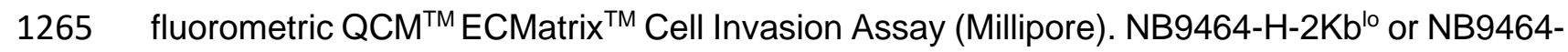
$1266 \mathrm{H}-2 \mathrm{~Kb}^{\text {hi }}$ cells in serum-free medium $\left(0.5 \times 10^{6}\right.$ cells $\left./ \mathrm{ml}\right)$ were added to the upper chamber 
Sengupta et al.

1267 and inserts (8 $\mu \mathrm{m}$ pore size) placed in the lower chamber containing medium with 10\% FBS.

1268 Following incubation at $37^{\circ} \mathrm{C}$ for $24 \mathrm{~h}$, cell invasion was measured according to 1269 manufacturer's instructions.

\section{EED226 treatment}

$12725 \times 10^{5} \mathrm{SH}-\mathrm{SY} 5 Y$ cells were seeded into $10 \mathrm{~cm}$ plates and treated with either $5 \mu \mathrm{M}$ EED226

1273 (Selleck Chemicals) or DMSO (vehicle control) for 6-8 days, following which samples were

1274 harvested for downstream analyses. Cells were replenished with fresh media containing DMSO

1275 or EED226 every 2 days.

\section{Compounds}

1278 Ceritinib (LDK378) and EED226 were purchased from Selleck Chemicals. Doxycycline and 1279 dimethyl Sulfoxide (DMSO) was purchased from Sigma-Aldrich.

\section{RNA extraction and q-PCR}

1282 Total RNA was isolated using the RNAeasy Mini kit (Qiagen). Purified RNA was reverse

1283 transcribed to cDNA using Superscript IV VILO master mix (Thermo Fisher Scientific) following

1284 the manufacturer's protocol. Quantitative PCR was performed using $1 \mu \mathrm{l}$ cDNA, 1x PowerTrack

1285 SYBR Green PCR master mix (Thermo Fisher Scientific) and PCR primers (200 nM) in a total

1286 volume of $25 \mu \mathrm{l}$ and analyzed on a ViiA 7 Real-Time PCR system (Thermo Fisher Scientific). Each

1287 individual biological sample was amplified in technical duplicate and normalized to GAPDH as an

1288 internal control. Relative expression was calculated according to the $2^{-\Delta \Delta \mathrm{CT}}$ quantification method

1289 (Livak and Schmittgen, 2001). PCR primer sequences are shown in Table S5. 
Sengupta et al.

\section{Synthetic RNA spike-in and RNA-sequencing}

1294 RNA-sequencing was performed on the following human NB cell lines: Kelly, NBL-S, CHP-212, 1295 SH-SY5Y, SH-SY5Y LDK-resistant and SH-EP. Biological duplicates $\left(5 \times 10^{6}\right.$ cells per replicate) 1296 were homogenized in $1 \mathrm{ml}$ of TRIzol Reagent (Invitrogen) and purified using the mirVANA miRNA 1297 isolation kit (Ambion) following the manufacturer's instructions. Total RNA was treated with DNA1298 free ${ }^{T M}$ DNase I (Ambion), spiked-in with ERCC RNA Spike-In Mix (Ambion) and analyzed on an 1299 Agilent 2100 Bioanalyzer (Agilent Technologies) for integrity. Sequencing libraries were prepared 1300 using LP-KAPA mRNA Hyper Prep and sequenced using Illumina HiSeq for 40 bases.

\section{Western blotting}

1303 Cells were homogenized in NP40 lysis buffer (Life Technologies) containing 1× cOmplete EDTA1304 free protease inhibitor cocktail and 1x PhosSTOP (Roche). Protein concentration was measured 1305 using the DC Protein Assay (Bio-Rad). $100 \mu \mathrm{g}$ total protein was denatured in LDS sample buffer 1306 (Invitrogen), separated on pre-cast 4-12\% Bis-Tris gels (Invitrogen) and transferred to 1307 nitrocellulose membranes (Bio-Rad). Membranes were blocked using 5\% dry milk (Sigma-Aldrich) 1308 in Tris-buffered saline (TBS) supplemented with $0.2 \%$ Tween-20 (TBS-T) for $1 \mathrm{hr}$, and incubated 1309 overnight with primary antibody in blocking buffer at $4{ }^{\circ} \mathrm{C}$. Chemiluminescent detection was 1310 performed with appropriate HRP-conjugated secondary antibodies and enhanced 1311 chemiluminescence reagents (Thermo Scientific). Images were developed using Genemate Blue 1312 ultra-autoradiography film (VWR).

\section{Antibodies}

1315 The following primary antibodies were used: MYCN (Cat \#51705), MYC (13987), GATA3 (5852),

1316 TAP1 (12341), TAP2 (12259), LMP7 (13635), NOTCH1 (3608), cleaved NOTCH1 (4147), SOX9 1317 (82630), AXL (8661), GAPDH (2118), $\beta$-actin (3700), IRF1 (8478), VIM (5741), YAP1 (4912), 
Sengupta et al.

TAZ1 (4883) (Cell Signalling Technologies (CST)); PHOX2B (Abcam;183741), FN1 (RnD systems; AF1918), LMP2 (Santa Cruz; 271354) and PRRX1 (Santa Cruz; 293386).

\section{Chromatin immunoprecipitation-quantitative PCR (ChIP-qPCR)}

Soluble chromatin was prepared as above from SH-SY5Y cells without or with dox-inducible PRRX1 expression (200 $\mathrm{ng} / \mathrm{ml}$ dox for 10 days). ChIP was performed as described in the preceding section using the following antibodies: H3K4me3 (Abcam 8580), H3K27me3 (Millipore 07-729), EZH2 (CST 5246), SUZ12 (CST 3737), EED (Millipore 17-10034), rabbit IgG (CST 2729). Purified ChIP DNA was dissolved in $60 \mu$ l of $1 \times$ TE. Quantitative PCR was performed on a ViiA 7 Real-Time PCR system (Thermo Fisher Scientific) with $1 \mu$ l purified DNA, 1x PowerTrack SYBR Green PCR master mix (Thermo Fisher Scientific) and PCR primers (200 nM) against the genomic regions of interest. Each individual biological sample was amplified in technical duplicate. Relative enrichment was quantified using the percent input method. PCR primer sequences are shown in Table S5.

\section{Chromatin immunoprecipitation-sequencing (ChIP-seq)}

Approximately $10-12 \times 10^{7}$ cells were crosslinked with $1 \%$ formaldehyde (Thermo Scientific) for $10 \mathrm{~min}$ at room temperature (RT) followed by quenching with $0.125 \mathrm{M}$ glycine for $5 \mathrm{~min}$. The cells were then washed twice in ice-cold 1x Phosphate Buffered Saline (PBS), and the cell pellet equivalent of $4 \times 10^{7}$ cells were flash frozen and stored at $-80^{\circ} \mathrm{C}$. Crosslinked cells were lysed in lysis buffer 1 (50 mM HEPES-KOH pH7.5, 140 mM NaCl, 1 mM EDTA, 10\% glycerol, 0.5\% NP40, $0.25 \%$ Triton X-100). The resultant nuclear pellet was washed once in lysis buffer 2 (10 mM Tris$\mathrm{HCl} \mathrm{pH} \mathrm{8,} 200 \mathrm{mM} \mathrm{NaCl}, 1 \mathrm{mM}$ EDTA, $0.5 \mathrm{mM}$ EGTA) and then resuspended in sonication buffer (50 mM HEPES-KOH pH 7.5, 140 mM NaCl, 1mM EDTA, 1mM EGTA, 1\% Triton X-100, 0.1\% sodium deoxycholate, $0.2 \%$ SDS). Chromatin was sheared using a Misonix 3000 sonicator (Misonix) and at the following settings: 10 cycles, each for $30 \mathrm{~s}$ on, followed by $1 \mathrm{~min}$ off, at a 
1344 power of approximately $20 \mathrm{~W}$. The lysates were then centrifuged for $15 \mathrm{~min}$ at $4{ }^{\circ} \mathrm{C}$, supernatants

1345 collected and diluted with an equal amount of sonication buffer without SDS to reach a final

1346 concentration of $0.1 \%$ SDS. For each ChIP, the chromatin equivalent of $1 \times 10^{7}$ cells was used.

$134750 \mu \mathrm{l}$ of Protein G Dynabeads per sample (Invitrogen) were blocked with $0.5 \%$ BSA (w/v) in $1 \mathrm{x}$

1348 PBS. Magnetic beads were loaded with the following antibodies: $10 \mu \mathrm{g}$ of H3K27me3 (Millipore

1349 07-729); $3 \mu \mathrm{g}$ of H3K27ac (Abcam 4729), and $3 \mu \mathrm{g}$ of H3K4me3 (Abcam 8580) and incubated

1350 overnight at $4^{\circ} \mathrm{C}$. The sonicated lysates were then incubated overnight at $4^{\circ} \mathrm{C}$ with the antibody-

1351 bound magnetic beads, washed with low-salt buffer (50 mM HEPES-KOH (pH 7.5), 0.1\% SDS,

$13521 \%$ Triton $\mathrm{X}-100,0.1 \%$ sodium deoxycholate, $1 \mathrm{mM}$ EGTA, $1 \mathrm{mM}$ EDTA, $140 \mathrm{mM} \mathrm{NaCl}$ and 1×

1353 complete protease inhibitor), high-salt buffer (50 mM HEPES-KOH (pH 7.5), 0.1\% SDS, 1\% Triton

$1354 \mathrm{X}-100,0.1 \%$ sodium deoxycholate, $1 \mathrm{mM}$ EGTA, $1 \mathrm{mM}$ EDTA, $500 \mathrm{mM} \mathrm{NaCl}$ and $1 \times$ complete

1355 protease inhibitor), $\mathrm{LiCl}$ buffer (20 mM Tris- $\mathrm{HCl}(\mathrm{pH} 8), 0.5 \% \mathrm{NP}-40,0.5 \%$ sodium deoxycholate,

$13561 \mathrm{mM}$ EDTA, $250 \mathrm{mM} \mathrm{LiCl}$ and $1 \times$ complete protease inhibitor) and Tris-EDTA (TE) buffer. DNA

1357 was then eluted in elution buffer (50 mM Tris- $\mathrm{HCl}(\mathrm{pH} 8.0), 10 \mathrm{mM}$ EDTA, 1\% SDS), and high-

1358 speed centrifugation performed to pellet the magnetic beads and collect the supernatants. The

1359 crosslinking was reversed overnight at $65^{\circ} \mathrm{C}$ in the presence of $300 \mathrm{mM} \mathrm{NaCl}$. RNA and protein

1360 were digested using RNase A and proteinase K, respectively, and DNA was purified with phenol-

1361 chloroform extraction and ethanol precipitation. Purified ChIP DNA was used to prepare Illumina

1362 multiplexed sequencing libraries using the NEBNext Ultra II DNA Library Prep kit and the

1363 NEBNext Multiplex Oligos for Illumina (New England Biolabs) according to the manufacturer's

1364 protocol. Libraries with distinct indices were multiplexed and run together on the Illumina NextSeq

1365500 (SY-415-1001, Illumina) for 75 base pairs.

\section{IFN-gamma induction and antigen presentation in NB9464 cells}

1368 Approximately $1 \times 10^{6}$ cells were seeded onto $10 \mathrm{~cm}$ plates. $24 \mathrm{hr}$ later, adherent cells were 1369 treated with recombinant mouse IFN- $\mathrm{Y}$ (Biolegend) for $24 \mathrm{~h}$ and harvested for $\mathrm{H}-2 \mathrm{~Kb}$ analysis 
Sengupta et al.

using FACS as described above. For antigen presentation assays, cells treated with IFN-y were

1371 pulsed with SIINFEKL (OVA peptide) at $37^{\circ} \mathrm{C}$. Cells were subsequently washed with $1 \mathrm{x}$ PBS to

1372 remove unbound peptide and processed for analysis using FACS.

\section{T cell activation assays}

1375 OT-I T cell receptor (TCR) transgenic mice were purchased from Jackson Laboratories (Bar 1376 Harbor, ME). Splenocytes were harvested and T cells were subsequently isolated from the

1377 mononuclear layer using Ficoll separation and directly used in co-culture assays. Successful 1378 enrichment of CD8 ${ }^{+}$T cells was confirmed by FACS analysis using the FITC-CD8 antibody. Cells 1379 were pulsed with SIINFEKL (OVA peptide) at $37^{\circ} \mathrm{C}$, to bind to cell surface $\mathrm{H}-2 \mathrm{~Kb}$. Cells were 1380 subsequently washed with $1 \mathrm{x}$ PBS to remove unbound peptide and then co-cultured with 1381 unstimulated OT-1 T cells for $24 \mathrm{~h}$. OT-I cells were then harvested, and sequentially stained with 1382 the Zombie NIR viability dye and FITC-CD8, PE-CD69 antibodies, followed by fixation with $1 \%$ 1383 paraformaldehyde (Polysciences, Inc). OT-1 cells were analyzed by flow cytometry using a 1384 FACSCanto II cell analyzer (Becton Dickinson) and FlowJo V10 software (Becton Dickinson).

In vitro assays for NKG2D binding

1387 SH-SY5Y parental, SH-SY5Y LDK-resistant, and SH-EP cells were incubated with recombinant 1388 human NKG2D-Fc chimeric protein or an equivalent concentration of human IgG, following which 1389 cells were washed, and sequentially stained with the Zombie NIR viability dye as described above 1390 followed by incubation with an Alexa 647-conjugated anti-human IgG antibody for 30 minutes. 1391 Cells were washed in FACS buffer and analyzed by flow cytometry using a FACSCanto II cell 1392 analyzer (Becton Dickinson) and FlowJo V10 software (Becton Dickinson). 
Sengupta et al.

NK cell degranulation assays

1397 Human peripheral blood NK cells were isolated from blood collars using a RosetteSep ${ }^{\mathrm{TM}}$ human

1398 NK cell enrichment cocktail (STEMCELL Technologies). NK cells were then co-cultured for $4 \mathrm{~h}$

1399 with confluent monolayers of SH-SY5Y parental, SH-SY5Y LDK-resistant, and SH-EP cells at in 1400 the presence of CD107a antibody (Biolegend). Additionally, co-cultures of NK cells and 721.221

1401 B cells were included as positive controls for degranulation. At the endpoint, NK cells were 1402 harvested, stained with Zombie Yellow (Biolegend) and CD56 FITC (or NKp46 AlexaFluor 647 ${ }^{\mathrm{TM}}$ ) 1403 and NKG2D PE (or mouse IgG1 PE) antibodies (Biolegend), followed by fixation with 1\% 1404 paraformaldehyde (Polysciences, Inc). For the NKG2D blocking assay, NK cells were incubated 1405 with purified anti-NKG2D antibody or mouse IgG1 isotype control at $37^{\circ} \mathrm{C}$, following which the 1406 degranulation assay was performed as detailed above. NK cells were analyzed by flow cytometry 1407 using a FACS Canto II (Becton Dickinson) and FlowJo V10 software (Becton Dickinson).

\section{Data sets}

1410 Publicly available RNA-seq data (GEO accession number GSE49711/GSE62564) from a cohort

1411 of 498 primary human neuroblastoma tumors, microarray expression data from 394 1412 neuroblastoma tumors (GSE120572) and 24 human neuroblastoma cell lines (GSE28019) were 1413 accessed through the R2 genomics analysis and visualization platform 1414 (https://hgserver1.amc.nl/cgi-bin/r2/). Clinical annotations for tumors were obtained from 1415 GSE49711/GSE62564 regarding MYCN status (MYCN-nonamplified vs. MYCN-amplified, INSS 1416 stage [high (stage 4) vs. low (1, 2, 3 and 4s], risk status (high vs. low) and age (< 18 months vs. $1417 \geq 18$ months). 
Sengupta et al.

\section{Analysis of RNA-sequencing data}

\section{RNA-seq data processing and identification of differentially expressed genes}

1424 Single-end RNA-seq samples with 40 base pair $(\mathrm{bp})$ read lengths were mapped to the human 1425 genome (GRCh38) and ERCC spike-in sequences. Reads were mapped to the genome using 1426 Bowtie2 (version 2.3.4.3) and default parameters. Reads that overlapped with the genomic 1427 location for exonic regions were used to calculate gene counts with the FeatureCounts (Subread 1428 package of version 1.6.3) package. Further, spike-in read counts were used for each sample to 1429 normalize the library sizes. These read counts were used to calculate the sample-specific size 1430 factor by using the function estimateSizeFactors (DESeq2) available in R. Normalized sample 1431 coverage profiles were then created from previously determined size factors by using 1432 bamCoverage (DeepTools v3.0.2) and parameters "--scaleFactor --skipNonCoveredRegions". To 1433 check the reproducibility of biological replicates for each condition, principal component analysis 1434 (PCA) and correlation (Spearman's rank coefficient) were assessed from the sample coverage 1435 profiles at genome-wide scale and visualized using scatterplots and heatmaps. Because these 1436 analyses showed a high correlation of sample coverage profiles between replicates, replicates 1437 were merged using samtools merge and processed again as described for the individual 1438 replicates. Next, differential gene expression analysis was performed using the DESeq2 in R. To 1439 detect differentially expressed genes (DEGs) in each sample, raw read counts from RNA-seq 1440 data were imported to the DESeq2 and the size factors calculated using the estimateSizeFactors

1441 function. A transcript with an absolute log2 fold-change $\geq 1.5$ and an adjusted $P$-value $\leq 0.01$ was 1442 considered significant.

\section{Enrichment analysis}

1445 Gene ontology enrichment for selected gene sets was performed by the Enrichr program 1446 (https://amp.pharm.mssm.edu/Enrichr/). All GO terms were ranked based on the Enrichr 1447 combined score, calculated by multiplying the adjusted $P$-value with the $z$-score using the Fisher's 
Sengupta et al.

1448

1449

1450

1451

\section{2}

1453

1454

1455

1456

1457

1458

1459

1460

1461

1462

1463

1464

1465

1466

1467

1468

1469

1470

1471

1472

1473

exact test. The Fisher's exact test was used to determine significant overlaps between the queried gene sets and other publicly available datasets. Enrichment of gene sets was considered significant for an adjusted $P$-value $\leq 0.01$, unless stated otherwise.

\section{Estimation of immune cell content in neuroblastoma tumors}

Cell type identification by estimating relative subsets (CIBERSORT) ${ }^{43}$, a deconvolution method was used to evaluate immune cell fractions from gene expression data using the $R$ package 'immunedeconv'. RNA transcript estimations were generated for all 498 neuroblastoma tumors using the LM22 signature matrix available for 22 immune cell types. CIBERSORT was run in "Absolute mode" with disabled quantile normalization as recommended for tumor RNA-seq data and the overall immune content produced by the algorithm compared among tumors.

\section{ChIP-seq analysis}

Data processing- All ChIP-seq raw datasets were processed as previously described ${ }^{83}$. The raw read quality of the samples was accessed using the Fastqc tool (v0.11.7) to identify possible sequencing errors and biases. Reads were aligned to the human genome (build hg19, GRCh37.75) using the mapper Bowtie (v2.3.4.3) with default parameters. Unique and nonduplicate reads that mapped to the reference genome were further processed using Samtools (v1.9) and the MarkDuplicates (v2.1.1) command of Picard tools. Next, antibody enrichment in each replicate as compared to input samples was verified using the PlotFingerprint command of deepTools (v3.1.1). Peak caller MACS2 (2.1.1) was used to identity narrow peaks (H3K4me3 and H3K27ac) with the parameters "--q 0.01--call-summits" and broad peaks (H3K27ac and H3K27me3) with the parameters “--broad-cutoff 0.01". Peaks that overlapped with black-listed regions (http://mitra.stanford.edu/kundaje/akundaje/release/blacklists/) of the reference genome (mostly comprised of major satellite repeats of telomeric and pericentromeric regions) were filtered out. Command "bamCompare" from the deepTools was used with the parameters "-- 
Sengupta et al.

1474 scaleFactorsMethod readCount --binSize 40 --operation subtract --smoothLength 80 --

1475 extendReads 200 " to create the input normalized bedgraph tracks for each replicate and

1476 afterwards negative values were set to zero and counts were scaled to reads per million/base pair

$1477(\mathrm{rpm} / \mathrm{bp})$ to account for differences in the library size. Bigwig files were created for visualization

1478 with bedGraphToBigWig. Subsequently, correlations among the ChIP-seq replicates were

1479 accessed using bigwigs with the command "multiBigwigSummary" from deepTools and highly

1480 correlated replicates merged at the BAM level. Peak identifications were then repeated in the

1481 same manner for these merged BAM files.

1483 Identification of super-enhancer regions- Super-enhancers (SEs) were identified using the

1484 ROSE algorithm (https://bitbucket.org/young computation/rose/src/master/). Briefly, H3K27ac

1485 binding regions identified by MACS2 as significant peaks, termed typical enhancers, were stitched

1486 together if they were within $12.5 \mathrm{~kb}$ of each other. These stitched enhancers were ranked by

1487 comparing the H3K27ac signal (density * length) with the input signal. The ROSE algorithm was

1488 used to determine the inclination point for all stitched H3K27ac signals and to segregate regular

1489 enhancers from SEs. To compare SEs in 5Y-parental, 5Y-LDK-resistant and SH-EP cells, the

1490 same maximum threshold was used between the conditions.

1491

1492 Analysis of histone binding changes between lineage states- To analyze the changes in 1493 occupancies of active (H3K27ac and H3K4me3) and repressive (H3K27me3) histone marks 1494 during the transition from adrenergic (5Y-par.) to mesenchymal (5Y-LDK-res., SHEP) states, we 1495 compared the peaks of histone marks identified by MACS2 at the promoter regions. For this 1496 purpose, we first extracted the promoter regions $\pm 2 \mathrm{~kb}$ with respect to the TSS (-2kb upstream 1497 to $+2 \mathrm{~kb}$ downstream) of all annotated protein coding genes and subsequently, retrieved the peaks 1498 of H3K27ac, H3K4me3 and H3K27me3 from 5Y-par., 5Y-LDK-res. and SH-EP cells. Now, to 1499 determine the differential binding of each histone mark between 5Y-par. and 5Y-LDK-res. cells, 
1500 we first combined all significant peaks called by MACS2 at the promoter regions and merged the

1501 peak regions that overlapped by at least 50\%. This 50\% threshold was used to avoid merging

1502 peaks that had clear and distinct summits. Next, the normalized active or repressive histone

1503 marks read densities were calculated for each region and a ratio of [log2 (5Y-LDK-res./ 5Y-par.)]

1504 was calculated. Shared peaks had similar enrichment of either active or repressive histone marks

1505 in both the cell types. Similar comparisons were made for active or repressive histone marks

1506 between 5Y-par. and SH-EP cells. To further compare changes in all histone marks at the

1507 promoters of immune genes, the gain of significant H3K27ac, H3K4me3 binding and loss of

1508 H3K27me3 signals were listed in mesenchymal (5Y-LDK-res., SHEP) cells as compared to 1509 adrenergic (5Y-par.) cells.

1510

1511 Integrated analysis of histone binding and gene expression- Cell-type specific differential

1512 enrichment of H3K27me3 and H3K4me3 binding in 5Y-par., 5Y-LDK-res. and SH-EP cells was

1513 determined by calculating the log2 $(\mathrm{H} 3 \mathrm{~K} 4 \mathrm{me} 3+1 / \mathrm{H} 3 \mathrm{~K} 27 \mathrm{me} 3+1)$ ratios in the promoter regions

1514 (TSS $\pm 2 \mathrm{~kb}$ ) of immune genes. Next, to examine the association between gene expression and

1515 differential enrichment of H3K27me3 and H3K4me3 binding in immune genes in 5Y-par., 5Y-LDK-

1516 res. and SH-EP cells, genes were ranked based on their expression values and plotted against

1517 the calculated ratios between the histone marks. 